\title{
The Milky Way Tomography with SDSS. III. Stellar Kinematics
}

\section{Citation}

Bond, Nicholas A., Zeljko Ivezic, Branimir Sesar, Mario Juric, Jeffrey A. Munn, Adam Kowalski, Sarah Loebman, et al. 2010. "The Milky Way Tomography with SDSS. III. Stellar Kinematics." The Astrophysical Journal 716 (1) (May 13): 1-29. doi:10.1088/0004-637x/716/1/1.

\section{Published Version}

doi:10.1088/0004-637X/716/1/1

\section{Permanent link}

http://nrs.harvard.edu/urn-3:HUL.InstRepos:33462900

\section{Terms of Use}

This article was downloaded from Harvard University's DASH repository, and is made available under the terms and conditions applicable to Other Posted Material, as set forth at http:// nrs.harvard.edu/urn-3:HUL.InstRepos:dash.current.terms-of-use\#LAA

\section{Share Your Story}

The Harvard community has made this article openly available.

Please share how this access benefits you. Submit a story.

\section{Accessibility}




\section{THE MILKY WAY TOMOGRAPHY WITH SDSS. III. STELLAR KINEMATICS}

Nicholas A. Bond ${ }^{1}$, Željko Ivezić $^{2}$, Branimir Sesar ${ }^{2}$, Mario Jurić ${ }^{3}$, Jeffrem A. Munn ${ }^{4}$, Adam Kowalski ${ }^{2}$, Sarah Loebman $^{2}$, Rok RošKar ${ }^{2}$, Timothy C. Beers ${ }^{5}$, Julianne Dalcanton ${ }^{2}$, Constance M. Rockosi ${ }^{6}$, Brian Yanny $^{7}$, Heidi J. Newberg ${ }^{8}$, Carlos Allende Prieto ${ }^{9,10}$, Ron Wilhelm ${ }^{11}$, Young Sun LeE ${ }^{5}$, Thirupathi Sivarani ${ }^{5,12}$, Steven R. Majewski ${ }^{13}$, John E. Norris ${ }^{14}$, Coryn A. L. Bailer-Jones ${ }^{15}$, Paola Re Fiorentin ${ }^{15,16}$, David SChlegel ${ }^{17}$, Alan Uomoto $^{18}$, Robert H. Lupton ${ }^{19}$, Gillian R. KNapp ${ }^{19}$, JAmes E. GunN ${ }^{19}$, Kevin R. Covey ${ }^{20}$, J. Allyn Smith ${ }^{21}$, Gajus Miknaitis ${ }^{7}$, Mamoru Doi ${ }^{22}$, Masayuki Tanaka ${ }^{23}$, Masataka Fukugita ${ }^{24}$, ${\text { Steve } \text { Kent }^{7} \text {, Douglas Finkbeiner }}^{20}$, Tom R. Quinn ${ }^{2}$, SuZanne Hawley ${ }^{2}$, Scott Anderson ${ }^{2}$, Furea Kiuchi ${ }^{2},{\text { Alex } \text { Chen }^{2}, \text { James Bushong }^{2} \text {, Harkirat Sohi }}^{2}$, Daryl Haggard ${ }^{2}$, Amy Kimball ${ }^{2}$, Rosalie McGurk ${ }^{2}$, John Barentine ${ }^{25}$, Howard Brewington ${ }^{25}$, Mike HarvaneK ${ }^{25}$, Scott Kleinman ${ }^{25}$, Jurek Krzesinski ${ }^{25}$, Dan Long ${ }^{25}$, Atsuko NitTa ${ }^{25}$, StePhanie SNedden ${ }^{25}$, Brian LeE $^{17}$, JefFrey R. Pier ${ }^{4}$, Hugh Harris ${ }^{14}$, Jonathan Brinkmann ${ }^{25}$, and Donald P. SChNeider ${ }^{26}$

${ }^{1}$ Physics and Astronomy Department, Rutgers University, Piscataway, NJ 08854-8019, USA

${ }^{2}$ Department of Astronomy, University of Washington, Box 351580, Seattle, WA 98195, USA

${ }^{3}$ Institute for Advanced Study, 1 Einstein Drive, Princeton, NJ 08540, USA

${ }^{4}$ U. S. Naval Observatory, Flagstaff Station, P.O. Box 1149, Flagstaff, AZ 86002, USA

${ }^{5}$ Department of Physics \& Astronomy and JINA: Joint Institute for Nuclear Astrophysics, Michigan State University, East Lansing, MI 48824, USA

${ }^{6}$ University of California-Santa Cruz, 1156 High Street, Santa Cruz, CA 95060, USA

${ }^{7}$ Fermi National Accelerator Laboratory, P.O. Box 500, Batavia, IL 60510, USA

${ }^{8}$ Department of Physics, Applied Physics, and Astronomy, Rensselaer Polytechnic Institute, 110 8th Street, Troy, NY 12180, USA

${ }^{9}$ McDonald Observatory and Department of Astronomy, University of Texas, Austin, TX 78712, USA

${ }^{10}$ Mullard Space Science Laboratory, University College London, Holmbury St. Mary, Dorking, Surrey, RH5 6NT, UK

${ }^{11}$ Department of Physics, Texas Tech University, Box 41051, Lubbock, TX 79409, USA

${ }^{12}$ Indian Institute of Astrophysics, Bangalore, 560034, India

${ }^{13}$ Department of Astronomy, University of Virginia, P.O. Box 400325, Charlottesville, VA 22904-4325, USA

${ }^{14}$ Research School of Astronomy \& Astrophysics, The Australian National University, Cotter Road, Weston, ACT 2611, Australia

${ }^{15}$ Max Planck Institut für Astronomie, Königstuhl 17, 69117 Heidelberg, Germany

${ }^{16}$ Department of Physics, University of Ljubljana, Jadranska 19, 1000 Ljubljana, Slovenia

${ }^{17}$ Lawrence Berkeley National Laboratory, One Cyclotron Road, MS 50R5032, Berkeley, CA 94720, USA

${ }^{18}$ Department of Physics and Astronomy, The John Hopkins University, 3701 San Martin Drive, Baltimore, MD 21218, USA

${ }^{19}$ Princeton University Observatory, Princeton, NJ 08544, USA

${ }^{20}$ Harvard-Smithsonian Center for Astrophysics, 60 Garden Street, Cambridge, MA 02138, USA

${ }^{21}$ Department of Physics \& Astronomy, Austin Peay State University, Clarksville, TN 37044, USA

${ }^{22}$ Institute of Astronomy, University of Tokyo, 2-21-1 Osawa, Mitaka, Tokyo 181-0015, Japan

${ }^{23}$ Department of Astronomy, Graduate School of Science, University of Tokyo, Hongo 7-3-1, Bunkyo-ku, Tokyo 113-0033, Japan

24 Institute for Cosmic Ray Research, University of Tokyo, Kashiwa, Chiba, Japan

${ }^{25}$ Apache Point Observatory, 2001 Apache Point Road, P.O. Box 59, Sunspot, NM 88349-0059, USA

${ }^{26}$ Department of Astronomy and Astrophysics, The Pennsylvania State University, University Park, PA 16802, USA Received 2009 August 31; accepted 2010 January 2; published 2010 May 13

\section{ABSTRACT}

We study Milky Way kinematics using a sample of 18.8 million main-sequence stars with $r<20$ and propermotion measurements derived from Sloan Digital Sky Survey (SDSS) and POSS astrometry, including 170,000 stars with radial-velocity measurements from the SDSS spectroscopic survey. Distances to stars are determined using a photometric-parallax relation, covering a distance range from $\sim 100 \mathrm{pc}$ to $10 \mathrm{kpc}$ over a quarter of the sky at high Galactic latitudes $\left(|b|>20^{\circ}\right)$. We find that in the region defined by $1 \mathrm{kpc}<Z<5 \mathrm{kpc}$ and $3 \mathrm{kpc}<R<$ $13 \mathrm{kpc}$, the rotational velocity for disk stars smoothly decreases, and all three components of the velocity dispersion increase, with distance from the Galactic plane. In contrast, the velocity ellipsoid for halo stars is aligned with a spherical coordinate system and appears to be spatially invariant within the probed volume. The velocity distribution of nearby $(Z<1 \mathrm{kpc}) \mathrm{K} / \mathrm{M}$ stars is complex, and cannot be described by a standard Schwarzschild ellipsoid. For stars in a distance-limited subsample of stars $(<100 \mathrm{pc})$, we detect a multi-modal velocity distribution consistent with that seen by HIPPARCOS. This strong non-Gaussianity significantly affects the measurements of the velocityellipsoid tilt and vertex deviation when using the Schwarzschild approximation. We develop and test a simple descriptive model for the overall kinematic behavior that captures these features over most of the probed volume, and can be used to search for substructure in kinematic and metallicity space. We use this model to predict further improvements in kinematic mapping of the Galaxy expected from Gaia and the Large Synoptic Survey Telescope.

Key words: Galaxy: disk - Galaxy: halo - Galaxy: kinematics and dynamics - Galaxy: stellar content - Galaxy: structure - methods: data analysis - stars: statistics

Online-only material: color figures

\section{INTRODUCTION}

The Milky Way is a complex and dynamic structure that is constantly being shaped by the infall of matter from the Local
Group and mergers with neighboring galaxies. From our vantage point inside the disk of the Milky Way, we have a unique opportunity to study an $\sim L^{*}$ spiral galaxy in great detail. By measuring and analyzing the properties of large numbers of 
individual stars, we can map the Milky Way in a ninedimensional space spanned by the three spatial coordinates, three velocity components, and three stellar parameters-luminosity, effective temperature, and metallicity.

In this paper, the third in a series of related studies, we use data obtained by the Sloan Digital Sky Survey (SDSS; York et al. 2000) to study in detail the distribution of tens of millions of stars in this multi-dimensional space. In Jurić et al. (2008, hereafter J08), we examined the spatial distribution of stars in the Galaxy, and in Ivezić et al. (2008a, hereafter I08) we extended our analysis to include the metallicity distribution. In this paper, working with a kinematic data set unprecedented in size, we investigate the distribution of stellar velocities. Our data include measurements from the SDSS astrometric, photometric, and spectroscopic surveys: the SDSS Data Release 7 (Abazajian et al. 2009) radial-velocity sample includes $\sim 170,000$ mainsequence stars, while the proper-motion sample includes 18.8 million stars, with about 6.8 million $F / G$ stars for which photometric-metallicity estimates are also available. These stars sample a distance range from $\sim 100 \mathrm{pc}$ to $\sim 10 \mathrm{kpc}$, probing much farther from Earth than the HIPPARCOS sample, which covers only the nearest $\sim 100$ pc (e.g., Dehnen \& Binney 1998; Nordström et al. 2004). With the SDSS data set, we are offered for the first time an opportunity to examine in situ the thin/thick disk and disk/halo boundaries over a large solid angle, using millions of stars.

In all three of the papers in this series, we have employed a set of photometric-parallax relations, enabled by accurate SDSS multi-color measurements, to estimate the distances to mainsequence stars. With these distances, accurate to $\sim 10 \%-15 \%$, the stellar distribution in the multi-dimensional phase space can be mapped and analyzed without any additional assumptions. The primary aim of this paper is thus to develop quantitative understanding of the large-scale kinematic behavior of the disk and halo stars. From the point of view of an observer, the goal is to measure and describe the radial-velocity and proper-motion distributions as functions of the position in, for example, the $r$ versus $g-r$ color-magnitude diagram, and as functions of the position of the analyzed sample on the sky. From the point of view of a theorist, we seek to directly quantify the behavior of the probability distribution function, $p\left(v_{R}, v_{\phi}, v_{Z} \mid R, \phi, Z, T, L,[\mathrm{Fe} / \mathrm{H}]\right)$, where $\left(v_{R}, v_{\phi}, v_{Z}\right)$ are the three velocity components in a cylindrical coordinate system, $(R, \phi, Z)$ describe the position of a star in the Galaxy, and $T$, $L$, and $[\mathrm{Fe} / \mathrm{H}]$ are its temperature, luminosity, and metallicity, respectively ("I" means "given").

This a different approach than that taken by the widely used "Besançon" Galaxy model (Robin \& Creze 1986; Robin et al. 2003, and references therein), which attempts to generate model stellar distributions from "first principles" (such as an adopted initial mass function) and requires dynamical self-consistency. Instead, we simply seek to describe the directly observed distributions of kinematic and chemical quantities without imposing any additional constraints. If these distributions can be described in terms of simple functions, then one can try to understand and model these simple abstractions, rather than the full voluminous data set.

As discussed in detail by J08 and I08, the disk and halo components have spatial and metallicity distributions that are well fitted by simple analytic models within the volume probed by SDSS (and outside regions with strong substructure, such as the Sgr dwarf tidal stream and the Monoceros stream). In this paper, we develop analogous models that describe the velocity distributions of disk and halo stars.
Questions we ask include the following:

1. What are the limitations of the Schwarzschild ellipsoidal approximation (a three-dimensional Gaussian distribution, Schwarzschild 1979) for describing the velocity distributions?

2. Given the increased distance range compared to older data sets, can we detect spatial variation of the best-fit Schwarzschild ellipsoid parameters, including its orientation?

3. Does the halo rotate on average?

4. Is the kinematic difference between disk and halo stars as remarkable as the difference in their metallicity distributions?

5. Do large spatial substructures, which are also traced in metallicity space, have distinctive kinematic behavior?

Of course, answers to a number of these questions are known to some extent (for excellent reviews, see Gilmore et al. 1989; Majewski 1993; Helmi 2008, for context and references, see also the first two papers in this series). For example, it has been known at least since the seminal paper of Eggen et al. (1962) that high-metallicity disk stars move on nearly circular orbits, while many low-metallicity halo stars move on eccentric, randomly oriented orbits. However, given the order of magnitude increase in the number of stars compared to previous work, larger distance limits, and accurate and diverse measurements obtained with the same facility, the previous results (see I08 for a summary of kinematic results) can be significantly improved and expanded.

The main sections of this paper include a description of the data and methodology (Section 2), followed by analysis of the various stellar subsamples. In Section 3, we begin by analyzing the proper-motion sample and determining the dependence of the azimuthal (rotational) and radial-velocity distributions on position for halo and disk subsamples selected along $l=0^{\circ}$ and $l=180^{\circ}$. The spectroscopic sample is used in Section 4 to obtain constraints on the behavior of the vertical-velocity component, and to measure the velocity-ellipsoid tilts. The resulting model is then compared to the full proper-motion sample and radial-velocity samples in Section 5. Finally, in Section 6, we summarize and discuss our results, including a comparison with prior results and other work based on SDSS data.

\section{DATA AND METHODOLOGY}

The characteristics of the SDSS imaging and spectroscopic data relevant to this work (Fukugita et al. 1996; Gunn et al. 1998, 2006; Hogg et al. 2001; Smith et al. 2002; Stoughton et al. 2002; Pier et al. 2003; Ivezić et al. 2004; Tucker et al. 2006; Abazajian et al. 2009; Yanny et al. 2009) are described in detail in the first two papers in the series (J08, I08). Here, we only briefly summarize the photometric-parallax and photometricmetallicity methods, and then describe the proper-motion data and their error analysis. The subsample definitions are described at the end of this section.

\subsection{The Photometric-parallax Method}

The majority of stars in the SDSS imaging catalogs ( $90 \%)$ are on the main sequence (J08 and references therein) and, using the broadband colors measured by SDSS, it is possible to estimate their absolute magnitude. Briefly, the $r$-band absolute magnitude, $M_{r}$, of a star can be estimated from its position 
on the stellar locus of the $M_{r}$ versus $g-i$ color-magnitude diagram. The position of this stellar locus is in turn sensitive to metallicity, so we must apply an additional correction to the absolute magnitude. A maximum-likelihood implementation of this method was introduced and discussed in detail in J08. The method was further refined by I08, who calibrated its dependence on metallicity using globular clusters.

We estimate absolute magnitudes using Equation (A7) in I08, which corrects for age effects, and Equation (A2) in the same paper to account for the impact of metallicity. The resulting distance range covered by the photometric-parallax relation depends upon color and metallicity, but spans $\sim 100 \mathrm{pc}$ to $\sim 10 \mathrm{kpc}$. Based on an analysis of stars in globular clusters, I08 estimate that the probable systematic errors in absolute magnitudes determined using these relations are about $0.1 \mathrm{mag}$, corresponding to $5 \%$ systematic distance errors (in addition to the $10 \%-15 \%$ random distance errors). In addition, Sesar et al. (2008, hereafter SIJ08) used a large sample of candidate wide-binary stars to show that the expected error distribution is mildly non-Gaussian, with a root-mean-square (rms) scatter in absolute magnitude of $\sim 0.3 \mathrm{mag}$. They also quantified biases in the derived absolute magnitudes due to unresolved binary stars.

\subsection{The Photometric-metallicity Method}

Stellar metallicity can significantly affect the position of a star in the color-magnitude diagram (there is a shift of $\sim 1 \mathrm{mag}$ between the median halo metallicity of $[\mathrm{Fe} / \mathrm{H}] \sim-1.5$ and the median disk metallicity of $[\mathrm{Fe} / \mathrm{H}] \sim-0.2)$. SDSS spectroscopy is only available for a small fraction of the stars in our sample, so we adopt a photometric-metallicity method based on SDSS $u-g$ and $g-r$ colors. This relation was originally calibrated by $\mathrm{I} 08$ using SDSS spectroscopic metallicities. However, the calibration of SDSS spectroscopic metallicity changed at the high-metallicity end after SDSS Data Release 6 (AdelmanMcCarthy et al. 2008). Therefore, we recalibrate their expressions as described in the Appendix. The new calibration, given in Equation (A1), is applicable to F/G stars with $0.2<g-r<0.6$ and has photometric-metallicity errors that approximately follow a Gaussian distribution with a width of 0.26 dex. In addition, the $\sim 0.1$ dex systematic uncertainties in SDSS spectroscopic metallicity (Beers et al. 2006; Allende Prieto et al. 2006, 2008; Lee et al. 2008a) are inherited by the photometric-metallicity estimator. We emphasize that photometric-metallicity estimates are only robust in the range $-2<[\mathrm{Fe} / \mathrm{H}]<0$ (see the Appendix for details).

For stars with $g-r>0.6$, we assume a constant metallicity of $[\mathrm{Fe} / \mathrm{H}]=-0.7$, motivated by results for the disk metallicity distribution presented in I08 and the fact that SDSS data are too shallow to include a large fraction of red halo stars. A slightly better approach would be to use the disk metallicity distribution from I08 to solve for best-fit distance iteratively, but the resulting changes in the photometric distances are negligible compared to other systematic errors.

\subsection{The SDSS-POSS Proper-motion Catalog}

We take proper-motion measurements from the Munn et al. (2004) catalog (distributed as a part of the public SDSS data releases), which is based on a comparison of astrometric measurements between SDSS and a collection of Schmidt photographic surveys. Despite the sizable random and systematic astrometric errors in the Schmidt surveys, the combination of a long baseline ( $\sim 50$ years for the POSS-I survey), and a recalibration of the photographic data using the positions of SDSS galaxies (see Munn et al. for details), results in median random proper-motion

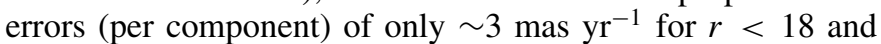

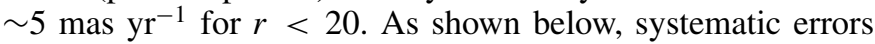
are typically an order of magnitude smaller. At a distance of

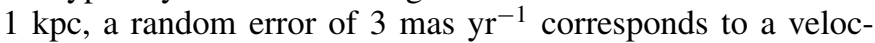
ity error of $\sim 15 \mathrm{~km} \mathrm{~s}^{-1}$, which is comparable to the radialvelocity accuracy delivered by the SDSS stellar spectroscopic survey $\left(\sim 5.3 \mathrm{~km} \mathrm{~s}^{-1}\right.$ at $g=18$ and $20 \mathrm{~km} \mathrm{~s}^{-1}$ at $g=20.3$; Schlaufman et al. 2009). At a distance of $7 \mathrm{kpc}$, a random error

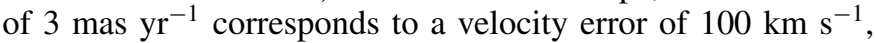
which still represents a usable measurement for large samples, given that systematic errors are much smaller $\left(\sim 20 \mathrm{~km} \mathrm{~s}^{-1}\right.$ at a distance of $7 \mathrm{kpc}$ ). The small and well-understood propermotion errors, together with the large distance limit and sample size (proper-motion measurements are available for about 38 million stars with $r<20$ from SDSS Data Release 7) make this catalog an unprecedented resource for studying the kinematics of Milky Way stars.

We warn the reader that proper-motion measurements made publicly available prior to SDSS Data Release 7 are known to have significant systematic errors. Here, we use a revised set of proper-motion measurements (Munn et al. 2008), which are publicly available only since Data Release 7. As described in the next section, we can assess the error properties of this revised proper-motion catalog using objects with known zero proper motion - that is, distant quasars.

\subsubsection{Determination of Proper-motion Errors Using Quasars}

All known quasars are sufficiently distant that their proper motions are vanishingly small compared to the expected random and systematic errors in the Munn et al. catalog. The large number of spectroscopically confirmed SDSS quasars (Schneider et al. 2007) which were not used in the recalibration of POSS astrometry can therefore be used to derive robust independent estimates of these errors. In SDSS Data Release 7 , there are 69,916 quasars with $14.5<r<20$, redshifts in the range $0.5<z<2.5$, and available proper motions (see the Appendix for the SQL query used to select and download the relevant data from the SDSS CAS). Within this sample of quasars,

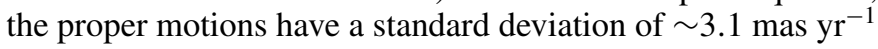
for each component (determined from the interquartile range), with medians differing from zero by less than 0.2 mas $\mathrm{yr}^{-1}$. The dependence of the random error on $r$-band magnitude is well-described by

$$
\sigma_{\mu}=2.7+2.0 \times 10^{0.4(r-20)} \operatorname{mas~yr}^{-1}
$$

fitting only to quasars in the range $15<r<20$. When the measurements of each proper-motion component are normalized by $\sigma_{\mu}$, the resulting distribution is approximately Gaussian, with only $\sim 1.8 \%$ of the quasar sample deviating by more than $3 \sigma$ from zero proper motion. In addition to their dependence on magnitude, the random proper-motion errors also depend on position on the sky, but the variation is relatively small $(\sim 20 \%$, see right panels in Figure 1). Finally, we find that the correlation between the errors in the two components is negligible compared to the total random and systematic errors.

The median proper motion for the full quasar sample is $\sim 0.2$ mas yr $^{-1}$, but the systematic errors can be larger by a factor of 2-3 in small sky patches, as illustrated in Figure 1. We find that the distribution of systematic proper-motion errors in $\sim 100 \mathrm{deg}^{2}$ 


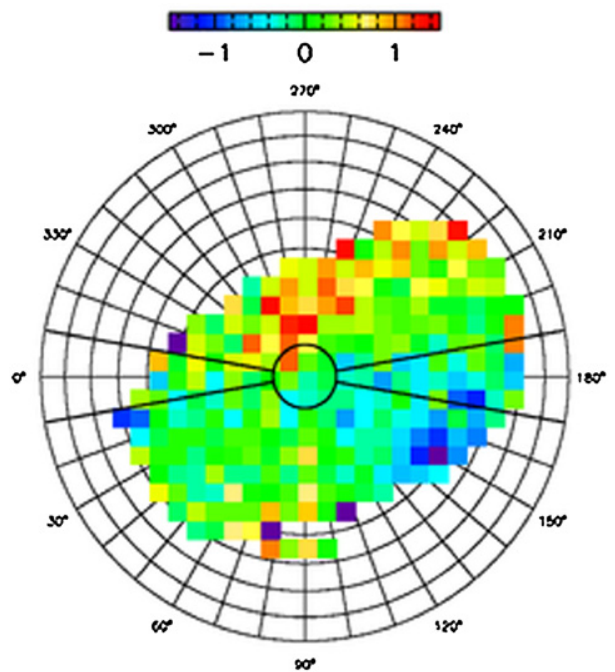

SOSS DR7 quosors ( $\mu_{1}$ medions: -1.5 to $1.5 \mathrm{mos} / \mathrm{yr}$ )

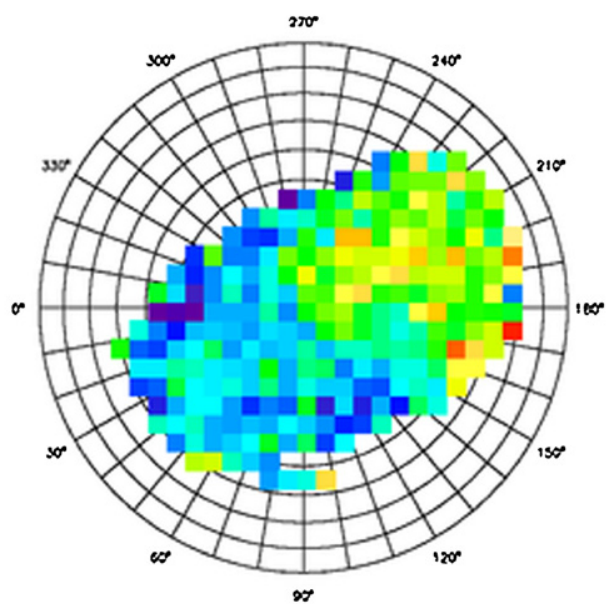

SOSS OR7 quosors ( $\mu_{0}$ medions: -1.5 to $1.5 \mathrm{mas} / \mathrm{yr}$ )

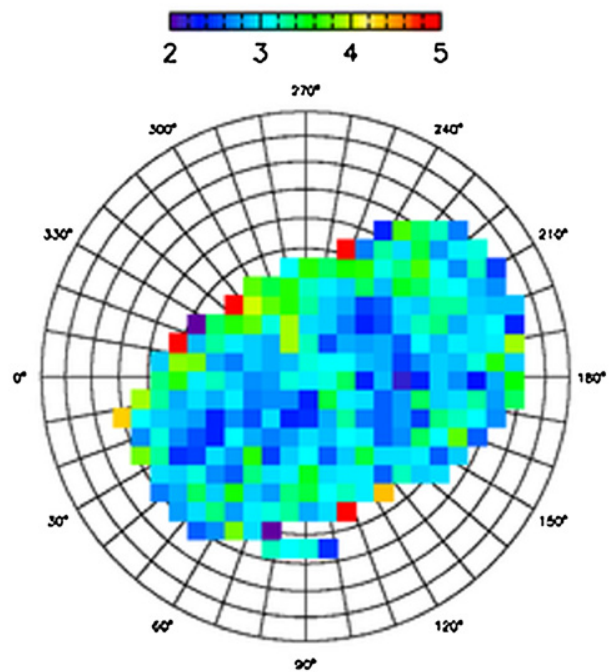

SDSS OR7 quosors (rms for $\mu_{7}: 2$ to $5 \mathrm{mos} / \mathrm{yr}$ )

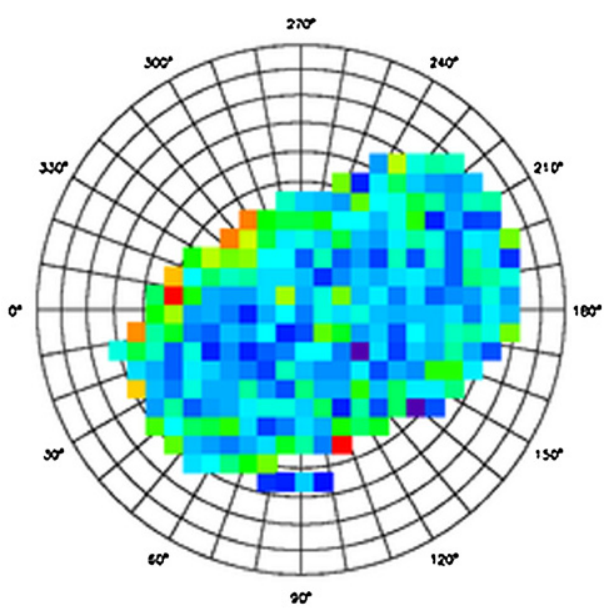

SDSS DR7 quosars (rms for $\mu_{0}: 2$ to $5 \mathrm{mos} / \mathrm{yr}$ )

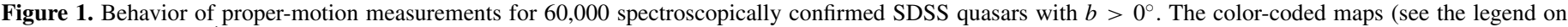

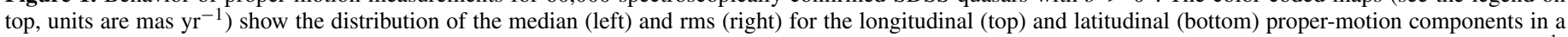

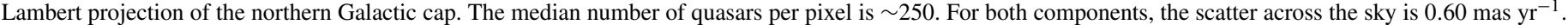

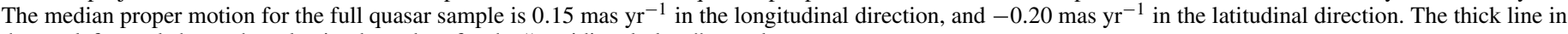
the top-left panel shows the selection boundary for the "meridional plane" sample.

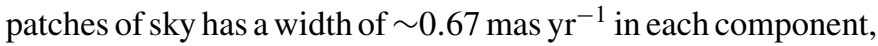
about twice as large as that expected from purely statistical noise (per bin, using Equation (1)). As the figure shows, a few regions of the sky have coherent systematic errors at a level

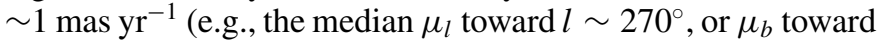
the inner Galaxy). Therefore, the kinematics measured using proper motions in these regions should be treated with caution.

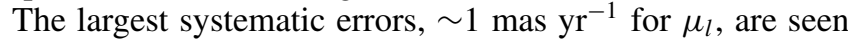
toward $l \sim 270^{\circ}$ in the top-left panel in Figure 1, which corresponds to $\delta \lesssim 10^{\circ}$. In this region, the systematic deviation of quasar proper motions from zero is approximately parallel to lines of constant right ascension, suggesting that the data may be suffering from systematic effects due to atmospheric refraction and spectral differences between quasars and galaxies used in the recalibration of POSS astrometry. This effect would be strongest for observations obtained at high air mass, as are typical for fields at low declination (the POSS data were obtained at a latitude of $\left.+33^{\circ}\right)$. We find that the median quasar proper motion in the $\delta$ direction is well-described by

$$
\left\langle\mu_{\delta}\right\rangle=-0.72+0.019 \delta \text { mas yr}^{-1}
$$

for $-5^{\circ}<\delta<30^{\circ}$, where $\delta$ is in degrees. At $\delta>30^{\circ}$, we find $\left\langle\mu_{\delta}\right\rangle \lesssim 0.2$ mas yr$^{-1}$.

The observed direction and magnitude of this systematic offset (corresponding to an astrometric displacement of up to $\sim 30$ mas) are consistent with detailed studies of atmospheric dispersion effects on observations of quasars (Kaczmarczik et al. 2009). Therefore, it is possible that the systematic errors in stellar proper motions (whose spectral energy distributions differ less from galaxy spectral energy distributions than is the case for quasars) are smaller than implied by Figure 1. Nevertheless, we will conservatively adopt the quasar propermotion distributions as independent estimates of systematic and random proper-motion errors for stars analyzed in this work.

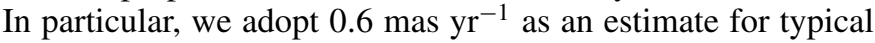
systematic proper-motion error.

The quasar sample has a much narrower color range than that seen in main-sequence stars $(96 \%$ of the quasar sample satisfies $-0.2<g-r<0.6$ ), and provides a better estimate of systematic proper-motion errors for the blue stars than for the red stars. Within the above well-sampled color range, we find a median proper-motion gradient with respect to the $g-r$ 
color of $\lesssim 0.1 \mathrm{mas} \mathrm{yr}^{-1} \mathrm{mag}^{-1}$ (per component). When the fit is extended to $g-r<1.6$ (using a much smaller number of

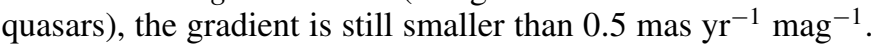
Hence, the proper-motion systematics have a color dependence that is smaller than, or at most comparable to, their dependence on sky position.

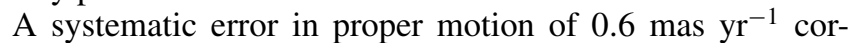
responds to a systematic velocity error of $3 \mathrm{~km} \mathrm{~s}^{-1}$ at $1 \mathrm{kpc}$, and $\sim 20 \mathrm{~km} \mathrm{~s}^{-1}$ at $7 \mathrm{kpc}$. In addition, the $\sim 5 \%$ systematic distance errors discussed in Section 2.1 are responsible for a $\sim 5 \%$ systematic velocity uncertainty. Hence, for a disk-like heliocentric tangential velocity of $20 \mathrm{~km} \mathrm{~s}^{-1}$, proper-motion systematics dominate at distances beyond $\sim 1 \mathrm{kpc}$. Similarly, for a halo-like heliocentric tangential velocity of $200 \mathrm{~km} \mathrm{~s}^{-1}$, proper-motion systematics will dominate at distances greater than $7 \mathrm{kpc}$. At smaller distances, the dominant systematic in our tangentialvelocity estimates comes from systematic distance errors. For most of the Galaxy volume analyzed in this work, the systematic distance errors dominate over systematic proper-motion errors.

\subsection{Comparison of Proper Motions with Independent Measurements}

As further tests of the proper-motion errors, we have analyzed two independent sets of measurements. As shown below, they confirm the results based on our analysis of the quasar sample.

We have compared the SDSS-POSS proper motions to proper-motion measurements by Majewski (1992) for a sample of 326 stars observed toward the North Galactic Pole. The measurements in the Majewski sample have random errors that are three times smaller, and comparable, but most likely, different systematic errors. The median proper-motion differences between the two data sets are below 1 mas $\mathrm{yr}^{-1}$, with an rms scatter 3-4 mas $\mathrm{yr}^{-1}$ (per coordinate). Hence, this comparison is consistent with our error estimates discussed in the preceding section, and with the estimates of Majewski (1992).

We have also compared the SDSS-POSS proper motions with proper motions from the SDSS stripe 82 region. In Bramich et al. (2008), proper motions are computed using only SDSS data, and thus they are expected to have different, and probably smaller, systematic errors than the SDSS-POSS proper motions (random errors for the stripe 82 proper motions are larger by about a factor of 2). For $~ 500,000$ stars with both SDSS-POSS and Bramich et al. proper-motion measurements, we find the median differences and the rms scatter to agree with expectation. A single worrisome result is that the median difference between the two data sets is a function of magnitude: we find a gradient of

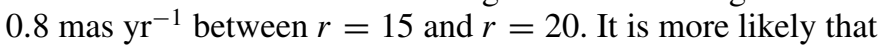
this gradient is due to systematic errors in centroiding sources on photographic plates, rather than a problem with SDSS data. This gradient corresponds to a systematic velocity error as a function of distance, $\Delta v \sim 4(D / \mathrm{kpc}) \mathrm{km} \mathrm{s}^{-1}$. For example, a halo star at $5 \mathrm{kpc}$, with a relative velocity of $200 \mathrm{~km} \mathrm{~s}^{-1}$, would have a systematic velocity uncertainty of $10 \%$. This systematic error is comparable to other sources of systematic errors discussed above, and has to be taken into account when interpreting our results below.

\subsection{The Main Stellar Samples}

When using proper motions, random errors in the inferred velocities have a strong dependence on magnitude, and therefore distance, while systematic errors are a function of position on the sky, as discussed above. Random errors in radial-velocity measurements also depend on magnitude, as fits to spectral features become more difficult at lower signal-to-noise ratios. As such, when radial-velocity and proper-motion measurements are analyzed simultaneously, the systematic and random errors combine in a complex way-care is needed when interpreting the results of such an analysis.

In order to minimize these difficulties, we separately analyze the proper-motion sample and the much smaller sample of stars with radial velocities. Furthermore, motivated by the metallicity distribution functions (MDFs) presented in I08, we separately treat the low-metallicity "halo" stars and the high-metallicity "disk" stars. For these two samples, we require $g-r<0.6$, the regime in which the photometric-metallicity estimator is believed to be accurate. Finally, we discuss a sample of "red" stars with $g-r>0.6$ (roughly, $g-i>0.8$ ), which are dominated by nearby $(<2 \mathrm{kpc})$ disk stars.

These samples are selected from SDSS Data Release 7 using the following common criteria:

1. unique unresolved sources that show subarcsecond parallax: binary processing flags DEBLENDED_AS_MOVING, SATURATED, BLENDED, BRIGHT, and NODEBLEND must be false, and parameter $\mathrm{nCHILD}=0$,

2. the interstellar extinction in the $r$ band, $A_{r}<0.3 \mathrm{mag}$,

3 . dust-corrected magnitudes in the range $14.5<r<$ $20 \mathrm{mag}$,

4. high galactic latitudes: $|b|>20^{\circ}$,

5. proper motion available,

yielding 20.1 million stars. The dust corrections, $A_{r}$, were computed using the Schlegel et al. (1998) dust maps, with conversion coefficients derived assuming an $R_{V}=3.1 \mathrm{dust}$ model. The intersection of the following color criteria then selects stars from the main stellar locus:

1. blue stars (6.9 million):

(a) $0.2<(g-r)<0.6$,

(b) $0.7<(u-g)<2.0$ and $-0.25<(g-r)-0.5(u-g)<$ 0.05 ,

(c) $-0.2<0.35(g-r)-(r-i)<0.10$;

2. red stars (11.9 million):

(a) $0.6<(g-r)<1.6$,

(b) $-0.15<-0.270 r+0.800 i-0.534 z+0.054<0.15$,

where the last condition is based on a "principal color" orthogonal to the stellar locus in the $i-z$ versus $r-i$ color-color diagram, as defined in Ivezić et al. (2004). This condition allows for a 0.15 mag offset from the stellar locus.

During the analysis, "blue" stars are often further divided by photometric metallicity (see below for details) into candidate "halo" stars $([\mathrm{Fe} / \mathrm{H}]<-1.1)$ and candidate "disk" stars $([\mathrm{Fe} / \mathrm{H}]>-0.9)$. Subsamples with intermediate metallicities include non-negligible fractions of both halo and disk stars. Although the reduced proper-motion diagram is frequently used for the separation and analysis of these two populations, we find it inadequate for our purposes; the vertical gradient in rotationalvelocity blurs the kinematic distinction between disk and halo (for a discussion, see SIJ08), and thus this method is applicable only to stars with significant proper motion (leading to severe selection effects). Although metallicities are not available for red stars, results from I08 imply that they are dominated by the disk population (red stars can only be seen out to $\sim 2 \mathrm{kpc}$ ).

For each of the subsamples defined above, we further separate those objects with SDSS spectroscopic data (see the Appendix 
for a sample SQL query) into independent subsamples. In total, these spectroscopic subsamples include 172,000 stars (out of 352,000 stars with spectra), after an additional requirement to select only main-sequence stars; that is, stars with $\log (g)>$ 3.5. ${ }^{27}$ Of the stars with spectroscopic data, 111,000 are blue $(0.2<g-r<0.6)$ and 61,000 are red $(0.6<g-r<1.6)$. When separating low- and high-metallicity stars with spectra, we use the spectroscopic metallicity (see Allende Prieto et al. 2006 for details). Due to increased difficulties with measuring $[\mathrm{Fe} / \mathrm{H}]$ for red stars $(g-r>0.6)$ from SDSS spectra, we adopt $[\mathrm{Fe} / \mathrm{H}]=-0.7$ for all such stars; this value is the median spectroscopic $[\mathrm{Fe} / \mathrm{H}]$ for stars with $0.6<g-r<1.3(\sigma=$ 0.4 dex). For over $90 \%$ of $\sim 30,000$ stars with $g-r>1.3$, $[\mathrm{Fe} / \mathrm{H}]$ is not successfully measured.

\subsection{Coordinate Systems and Transformations}

Following J08 and I08, we use a right-handed, Cartesian Galactocentric coordinate system defined by the following set of coordinate transformations:

$$
\begin{gathered}
X=R_{\odot}-D \cos (l) \cos (b) \\
Y=-D \sin (l) \cos (b) \\
Z=D \sin (b),
\end{gathered}
$$

where $R_{\odot}=8 \mathrm{kpc}$ is the adopted distance to the Galactic center, $D$ is distance of the star from the Sun, and $(l, b)$ are the Galactic coordinates. Note that the $Z=0$ plane passes through the Sun, not the Galactic center (see J08), the $X$-axis is oriented toward $l=180^{\circ}$, and the $Y$-axis is oriented toward $l=270^{\circ}$ (the disk rotates toward $l \sim 90^{\circ}$ ). The main reason for adopting a Galactocentric coordinate system, rather than a traditional heliocentric system, is that new data sets extend far beyond the solar neighborhood.

We also employ a cylindrical coordinate system defined by

$$
R=\sqrt{X^{2}+Y^{2}}, \quad \phi=\tan ^{-1}\left(\frac{Y}{X}\right) .
$$

The tangential velocity, $v$, is obtained from the proper motion, $\mu$, and the distance $D$ by

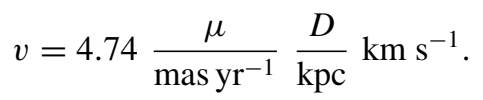

Given the line-of-sight radial velocity, $v_{\text {rad }}$, and the two components of tangential velocity aligned with the Galactic coordinate system, $v_{l}$ and $v_{b}$, the observed heliocentric Cartesian velocity components are given by

$$
\begin{gathered}
v_{X}^{\mathrm{obs}}=-v_{\mathrm{rad}} \cos (l) \cos (b)+v_{b} \cos (l) \sin (b)+v_{l} \sin (l) \\
v_{Y}^{\mathrm{obs}}=-v_{\mathrm{rad}} \sin (l) \cos (b)+v_{b} \sin (l) \sin (b)-v_{l} \cos (l) \\
v_{Z}^{\mathrm{obs}}=v_{\mathrm{rad}} \sin (b)+v_{b} \cos (b) .
\end{gathered}
$$

These components are related to the traditional $U V W$ nomenclature by, $v_{X}=-U, v_{Y}=-V$, and $v_{Z}=W$, e.g., Binney \& Merrifield (1998).

In order to obtain the Galactocentric cylindrical velocity components, we must first correct for the solar motion. Taking into account $\mathrm{H}$ I measurements of the Galactic rotation curve

\footnotetext{
27 Note that the majority of stars with $g-r>1.2$ do not have reliable measurements of $\log (g)$-we assume that all stars with $g-r>1.2$ are main-sequence stars.
}

(Gunn et al. 1979) and Hipparcos measurements of Cepheid proper motions (Feast \& Whitelock 1997), we adopt $v_{\mathrm{LSR}}=$ $220 \mathrm{~km} \mathrm{~s}^{-1}$ for the motion of the local standard of rest and $R_{\odot}=8 \mathrm{kpc}$ (for an analysis of other recent measurements, see Bovy et al. 2009). The adopted value of $R_{\odot}$ is motivated by geometrical measurements of the motions of stars around Sgr A*, which yield $R_{\odot}=7.94 \pm 0.42 \mathrm{kpc}$ (Eisenhauer et al. 2003). For the solar peculiar motion, we adopt the HIPPARCOS result, $v_{X}^{\odot, \text { pec }}=-10.0 \pm 0.4 \mathrm{~km} \mathrm{~s}^{-1}, v_{Y}^{\odot, \text { pec }}=-5.3 \pm 0.6 \mathrm{~km} \mathrm{~s}^{-1}$, and $v_{Z}^{\odot, \mathrm{pec}}=7.2 \pm 0.4 \mathrm{~km} \mathrm{~s}^{-1}$ (Dehnen \& Binney 1998, also see Hogg et al. 2005). Using these values, along with Equation (6), we obtain the Galactocentric velocity components:

$$
v_{i}=v_{i}^{\mathrm{obs}}+v_{i}^{\odot}, \quad i=X, Y, Z,
$$

where $v_{X}^{\odot}=-10 \mathrm{~km} \mathrm{~s}^{-1}, v_{Y}^{\odot}=-225 \mathrm{~km} \mathrm{~s}^{-1}$, and $v_{Z}^{\odot}=$ $7 \mathrm{~km} \mathrm{~s}^{-1}$ (note that $v_{Y}^{\odot}=-v_{\mathrm{LSR}}+v_{Y}^{\odot, \mathrm{pec}}$ ). Below, we discuss attempts to directly determine the solar peculiar motion (Section 4.2) and $v_{\text {LSR }}$ (Section 5.3) from our data.

Finally, the cylindrical components, $v_{R}$ and $v_{\phi}$, can be computed using a simple coordinate rotation,

$$
\begin{gathered}
v_{R}=v_{X} \frac{X}{R}+v_{Y} \frac{Y}{R} \\
v_{\phi}=-v_{X} \frac{Y}{R}+v_{Y} \frac{X}{R} .
\end{gathered}
$$

Note that, in our adopted system, the disk has a prograde rotation $v_{\phi}=-220 \mathrm{~km} \mathrm{~s}^{-1}$; retrograde rotation is indicated by $v_{\phi}>0$. Stars with $v_{R}>0$ move away from the Galactic center, and stars with $v_{Z}>0$ move toward the North Galactic Pole.

\subsection{Analysis Philosophy}

Such a massive data set, extending to a large distance limit and probing a large fraction of the Galaxy volume, can be used to map the kinematics of stars in great detail. It can also be used to obtain best-fit parameters of an appropriate kinematic model. However, it is not obvious what model (functional form) to chose without at least some preliminary analysis. Hence, in the next two sections, we first discuss various projections of the multi-dimensional space of the available observable quantities and obtain a number of constraints on the spatial variation of stellar kinematics. We then synthesize all of the constraints into a model described in Section 5. Before proceeding with our analysis, we provide a brief summary of the first two papers in this series, whose results inform our subsequent analysis.

\subsection{A Summary of Papers I and II}

Using photometric data for 50 million stars from SDSS Data Release 4 (Adelman-McCarthy et al. 2006), sampled over a distance range from $100 \mathrm{pc}$ to $15 \mathrm{kpc}, \mathrm{J} 08$ showed that the stellar number density distribution, $\rho(R, Z, \phi)$ can be well-described (apart from local overdensities; the J08 best fit was refined using residual minimization algorithms) as a sum of two cylindrically symmetric components:

$$
\rho(R, Z, \phi)=\rho_{D}(R, Z)+\rho_{H}(R, Z) .
$$

The disk component can be modeled as a sum of two exponential disks

$$
\rho_{D}(R, Z)=\rho_{D}\left(R_{\odot}\right)\left(e^{-\frac{\left|Z+Z_{\odot}\right|}{H_{1}}-\frac{\left(R-R_{\odot}\right)}{L_{1}}}+\epsilon_{D} e^{-\frac{\left|Z+Z_{\odot}\right|}{H_{2}}-\frac{\left(R-R_{\odot}\right)}{L_{2}}}\right),
$$


while the halo component requires an oblate power-law model

$$
\rho_{H}(R, Z)=\rho_{D}\left(R_{\odot}\right) \epsilon_{H}\left(\frac{R_{\odot}^{2}}{R^{2}+\left(Z / q_{H}\right)^{2}}\right)^{n_{H} / 2} .
$$

The best-fit parameters are discussed in detail by J08. We have adopted the following values for the parameters relevant to this work (second column in Table 10 from J08): $Z_{\odot}=$ $25 \mathrm{pc}, H_{1}=245 \mathrm{pc}, H_{2}=743 \mathrm{pc}, \epsilon_{D}=0.13, \epsilon_{H}=0.0051$, $q_{H}=0.64$, and $n_{H}=2.77$. The normalization $\rho_{D}\left(R_{\odot}\right)$ (which is essentially the local luminosity function for main-sequence stars) is listed in $\mathrm{J} 08$ as a function of color.

Using a photometric-metallicity estimator for $\mathrm{F} / \mathrm{G}$ mainsequence stars, I08 obtained an unbiased, three-dimensional metallicity distribution of $\sim 2.5$ million $\mathrm{F} / \mathrm{G}$ stars at heliocentric distances of up to $\sim 8 \mathrm{kpc}$. They found that the MDFs of the halo and disk stars are clearly distinct. The median metallicity of the disk exhibits a vertical (with respect to the Galactic plane, $Z$ ) gradient, and no gradient in the radial direction (for $Z>0.5 \mathrm{kpc}$ and $6<R<10 \mathrm{kpc}$ ).

Similarly to the stellar number density distribution, $\rho(R, Z)$, the overall behavior of the MDF $p([\mathrm{Fe} / \mathrm{H}] \mid R, Z)$ for disk stars can be well-described as a sum of two components

$$
\begin{aligned}
p(x=[\mathrm{Fe} / \mathrm{H}] \mid R, Z, \phi)= & {\left[1-f_{H}(R, Z)\right] p_{D}(x \mid Z) } \\
& +f_{H}(R, Z) p_{H}(x),
\end{aligned}
$$

where the halo star-count ratio is simply,

$$
f_{H}(R, Z)=\frac{\rho_{H}(R, Z)}{\rho_{D}(R, Z)+\rho_{H}(R, Z)} .
$$

The halo metallicity distribution, $p_{H}([\mathrm{Fe} / \mathrm{H}])$, is spatially invariant within the probed volume, and well-described by a Gaussian distribution centered on $[\mathrm{Fe} / \mathrm{H}]=-1.46$, with an intrinsic (corrected for measurement errors) width $\sigma_{H}=$ 0.30 dex. For $|Z| \lesssim 10 \mathrm{kpc}$, an upper limit on the halo radial metallicity gradient is 0.005 dex $\mathrm{kpc}^{-1}$.

The disk metallicity distribution varies with $Z$ such that its shape remains fixed, while its median, $\mu_{D}$, varies as

$$
\mu_{D}(Z)=\mu_{\infty}+\Delta_{\mu} e^{-\frac{|Z|}{H_{\mu}}},
$$

with the best-fit parameter values $H_{\mu}=0.5 \mathrm{kpc}, \mu_{\infty}=-0.82$, and $\Delta_{\mu}=0.55$. The shape of the disk metallicity distribution can be modeled as

$$
\begin{aligned}
p_{D}(x=[\mathrm{Fe} / \mathrm{H}] \mid Z)= & 0.63 G[x \mid \mu=a(Z), \sigma=0.2] \\
& +0.37 G[x \mid \mu=a(Z)+0.14, \sigma=0.2],
\end{aligned}
$$

where the position $a$ and the median $\mu_{D}$ are related via $a(Z)=\mu_{D}(Z)-0.067$ (unless measurement errors are very large).

The main result of this third paper in the series is the extension of these results for number density and metallicity distributions to include kinematic quantities.

\section{ANALYSIS OF THE PROPER-MOTION SAMPLE}

We begin by analyzing the proper-motion measurements of stars observed toward the North Galactic Pole. In this region, the Galactocentric azimuthal velocity, $v_{\phi}$, and radial velocity,
$v_{R}$, can be determined directly from the proper-motion measurements (that is, without knowledge of the spectroscopically determined radial velocity, $v_{\text {rad }}$ ). In this way, we can study the kinematic behavior of stars as a function of metallicity and distance from the Galactic plane, $Z$. We then extend our analysis to the entire meridional $Y=0$ plane, and study the variation of stellar kinematics with $R$ and $Z$. In the following section, we only consider the northern Galactic hemisphere, where most of the proper-motion data are available.

\subsection{Kinematics Toward the North Galactic Pole}

We select three stellar subsamples in the region $b>80^{\circ}$, including 14,000 blue disk stars at $Z<7 \mathrm{kpc}, 23,000$ blue halo stars at $Z<7 \mathrm{kpc}$, and a sample of 105,000 red stars at $Z<1 \mathrm{kpc}$. In Figure 2, we plot the distribution of $v_{\phi}$ versus $v_{R}$ for $\sim 6000$ blue disk and halo stars at $Z=4-5 \mathrm{kpc}$. In this and all subsequent two-dimensional projections of the velocity distribution we plot smoothed, color-coded maps, where the velocity distributions are estimated using the Bayesian density estimator of Ivezić et al. (2005, see their Appendix for the derivation and a discussion). At a given position, the density is evaluated as

$$
\rho=\frac{C}{\sum_{i=1}^{N} d_{i}^{2}},
$$

where $d_{i}$ is the distance in the velocity-velocity plane, and we sum over the $N=10$ nearest neighbors. The normalization constant, $C$, is easily evaluated by requiring that the density summed over all pixels is equal to the total number of data points divided by the total area. The grid size is arbitrary, but the map resolution is determined by the density of points-we choose pixel size equal to one half of the mean velocity error. As shown by Ivezić et al. (2005), this method is superior to simple Gaussian smoothing. For comparison, we also plot linearly spaced density contours.

The six panels of Figure 2 demonstrate the variation of kinematics with metallicity, with the full range of metallicities $(-3<[\mathrm{Fe} / \mathrm{H}]<0)$ plotted in the upper left panel and subsamples with increasing metallicity running from left to right, top to bottom. The mean azimuthal velocity varies strongly with metallicity, from a non-rotating low-metallicity subsample with large velocity dispersion (top center panel) to a rotating high-metallicity sample with much smaller dispersion (bottomright panel). This strong metallicity-kinematic correlation is qualitatively the same as discussed in the seminal paper by Eggen et al. (1962), except that here it is reproduced in situ with a 100 times larger, nearly complete sample, thus extending it beyond the solar neighborhood. There are some indications of substructure in the velocity distribution, but much of it remains unresolved due to the large velocity-measurement errors.

The substructure becomes more apparent in Figure 3, where we plot the same velocity-space projection for 60,000 stars within $Z<2.5 \mathrm{kpc}$. In this figure, the panels show subsamples of increasing distance from the Galactic plane, beginning with $Z=0.1-0.2 \mathrm{kpc}$ in the upper left panel (note the changing axes between the top and bottom rows). The substructure seen in the closest bin probed by red stars is very similar to the substructure seen in the local HIPPARCOS sample (Dehnen 1998). These results were based on a maximum-likelihood analysis over the entire sky, while our result arises from a direct mapping of the velocity distribution of stars selected from a small region $\left(\sim 300 \mathrm{deg}^{2}\right)$. Using a subsample of $\sim 17,000$ 



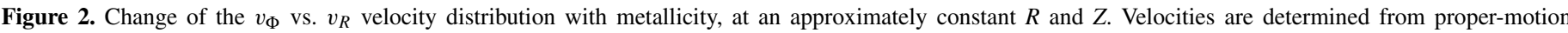

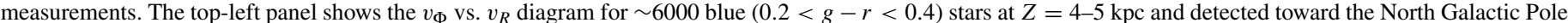

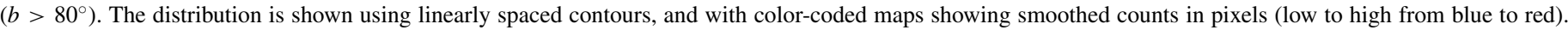

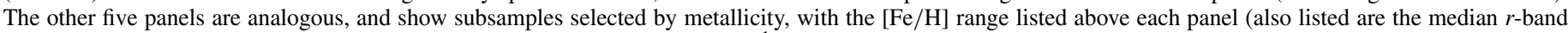
magnitude and subsample size). The measurement errors are typically $70 \mathrm{~km} \mathrm{~s}^{-1}$ (per star). Note the strong variation of median $v_{\Phi}$ with metallicity.

(A color version of this figure is available in the online journal.)

HIPPARCOS stars with full three-dimensional velocity information, Nordström et al. (2004), Famaey et al. (2005), and Holmberg et al. $(2007,2009)$ have detected the same kinematic morphology. The similarity between these HIPPARCOS-based velocity distributions and ours, including the multi-modal behavior reminiscent of moving groups (Eggen 1996), is quite encouraging, given the vastly different data sources. The similarity of observed substructure with moving groups is even more striking for stars from a closer distance bin $(Z=50-100 \mathrm{pc})$, matched to distances probed by the HIPPARCOS sample (see Figure 4). As suggested by De Simone et al. (2004), these moving groups may arise from irregularities in the Galactic gravitational potential.

The remainder of our analysis will focus on blue stars, which sample a much larger distance range. For a detailed study of the velocity distribution of nearby red stars, including a discussion of non-Gaussianity, vertex deviations, and difficulties with traditional thin/thick-disk separation, we refer the reader to A. Kowalski et al. (2010, in preparation).

The dependence of the rotational velocity on height above the Galactic plane is shown in Figure 5. The two subsamples display remarkably different kinematic behavior (first seen locally by Eggen et al. 1962) with halo stars exhibiting a small constant rotational motion $\left(\sim-20 \mathrm{~km} \mathrm{~s}^{-1}\right)$, and disk stars exhibiting a large rotational-velocity component $\left(\sim-200 \mathrm{~km} \mathrm{~s}^{-1}\right.$ at $Z \sim$ $1 \mathrm{kpc}$ ) that decreases with height above the Galactic plane.

We have performed the same analysis using proper motions based only on POSS data, with SDSS positions not included in the proper-motion fit (not publicly available ${ }^{28}$ ). While random proper-motion errors become larger when SDSS data are not used, the median rotational velocity for halo stars decreases to only $5 \mathrm{~km} \mathrm{~s}^{-1}$, suggesting that the apparent rotational motion in the halo subsample is influenced by systematic errors. These tests also suggest that the leading contribution to systematic proper-motion errors could be a difference between the SDSS (digital data) and POSS (digitized photographic data) centroiddetermination algorithms. In addition, Smith et al. (2009) did not detect halo rotation using a smaller sample, but with more robust proper-motion measurements based on only SDSS data, while Allende Prieto et al. (2006) found no evidence for halo rotation using SDSS DR3 radial velocities. We conclude that the net halo rotation in the direction of the North Galactic Pole is $\left|v_{\text {rot }}\right| \lesssim 10 \mathrm{~km} \mathrm{~s}^{-1}$. In addition, the measured halo velocity dispersion increases with $Z$, but when random measurement errors are taken into account, the data are consistent with a constant dispersion of $\sigma_{\phi}^{H}=85 \pm 5 \mathrm{~km} \mathrm{~s}^{-1}$ (derived using the test described in Section 5).

The decrease of rotational velocity with $Z$ for disk stars (often referred to as asymmetric drift, velocity lag, or velocity shear; see Section 3.4 of I08 for more details and references to related work) is in agreement with a preliminary analysis presented in I08. We find that the observed behavior in the $Z=1-4 \mathrm{kpc}$

\footnotetext{
28 Available from J. Munn on request.
} 

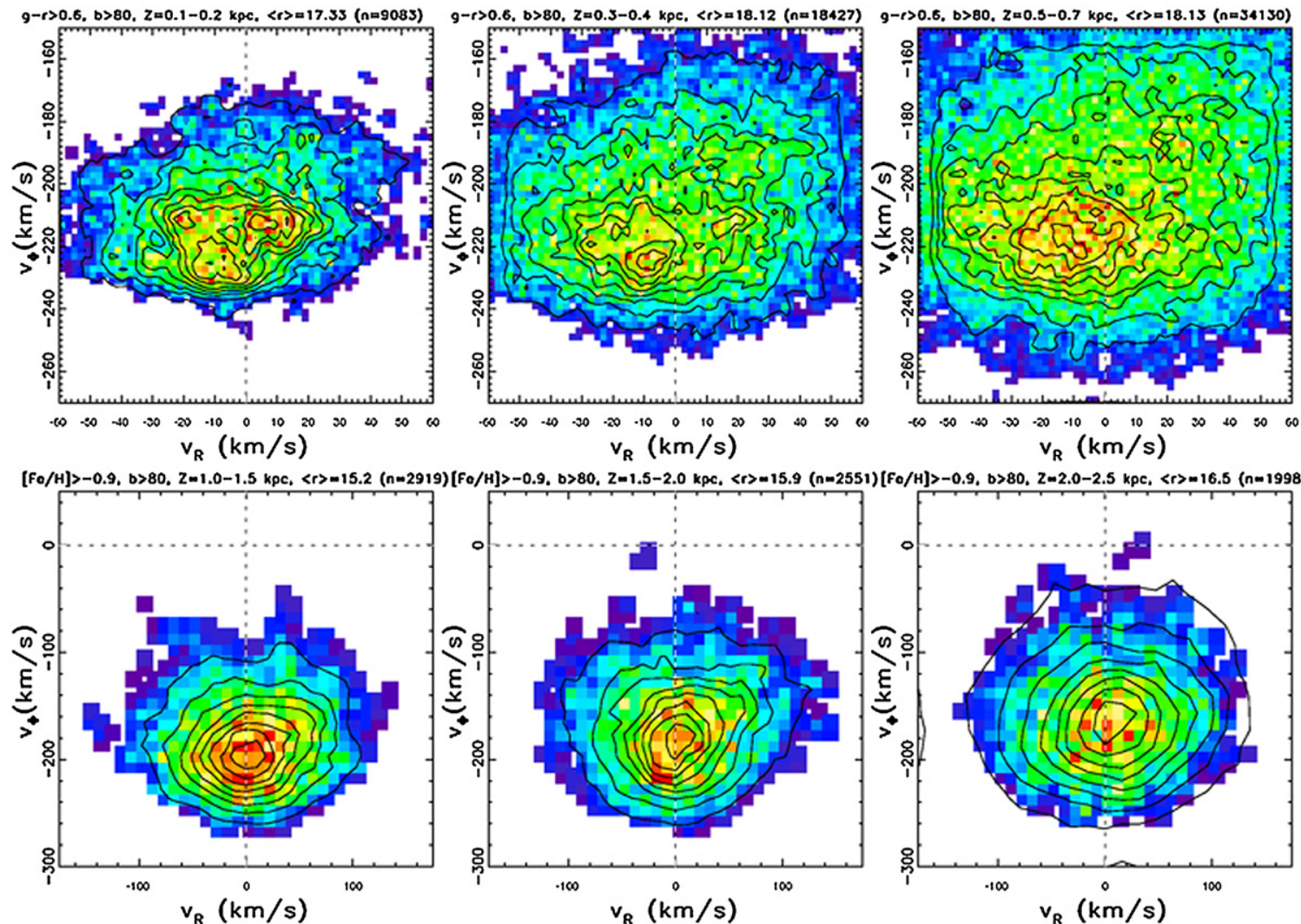

Figure 3. Similar to Figure 2, except that the $v_{\Phi}$ vs. $v_{R}$ velocity distribution is plotted for a range of $Z$. The top row shows the $v_{\Phi}$ vs. $v_{R}$ diagrams for $\sim 60,000$ red $(g-r>0.6)$ stars at $Z=100-700 \mathrm{pc}$ and observed toward the North Galactic Pole. Each panel corresponds to a narrow range in $Z$, given above each panel. The measurement errors vary from $\sim 3 \mathrm{~km} \mathrm{~s}^{-1}$ in the closest bin to $\sim 12 \mathrm{~km} \mathrm{~s}^{-1}$ in the most distant bin. Note the complex multi-modal substructure in the top-left panel. The bottom three panels are analogous, and show the $v_{\Phi}$ vs. $v_{R}$ diagrams for $\sim 7000$ blue $(0.2<g-r<0.4)$ stars with high metallicity ([Fe/H] $\left.>-0.9\right)$. The measurement errors vary from $\sim 20 \mathrm{~km} \mathrm{~s}^{-1}$ in the closest bin to $\sim 35 \mathrm{~km} \mathrm{~s}^{-1}$ in the most distant bin. Note that the median $v_{\Phi}$ approaches zero as $Z$ increases.

(A color version of this figure is available in the online journal.)

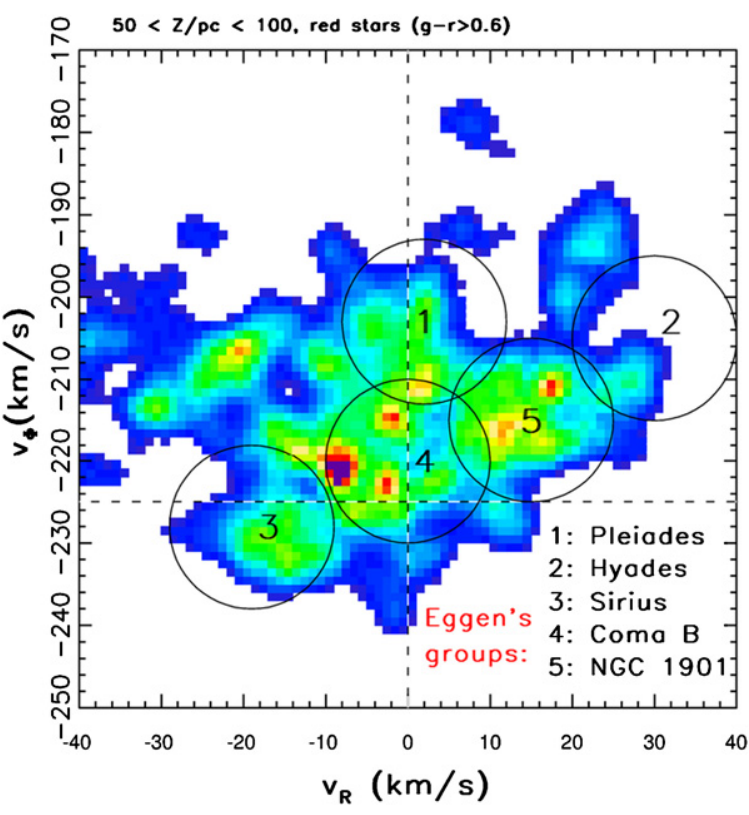

Figure 4. Similar to the top-left panel in Figure 3, except that stars are selected from a distance bin that corresponds to HIPPARCOS sample ( $Z=50-100 \mathrm{pc})$. The positions of Eggen's moving groups (Eggen 1996) are marked by circles, according to the legend in the bottom-right corner. The horizontal line at $v_{\phi}=-225 \mathrm{~km} \mathrm{~s}^{-1}$ corresponds to vanishing heliocentric motion in the rotational direction.

(A color version of this figure is available in the online journal.) range can be described by

$$
\left\langle v_{\phi}\right\rangle=-205+19.2\left|\frac{Z}{\mathrm{kpc}}\right|^{1.25} \mathrm{~km} \mathrm{~s}^{-1}
$$

The measured rotational-velocity dispersion of disk stars increases with $Z$ faster than can be attributed to measurement errors. Using a functional form $\sigma=a+b|Z|^{c}$, we obtain an intrinsic velocity dispersion fit of

$$
\sigma_{\phi}^{D}=30+3.0\left|\frac{Z}{\mathrm{kpc}}\right|^{2.0} \mathrm{~km} \mathrm{~s}^{-1} .
$$

This function and the best-fit rotational velocity for halo stars are shown as dotted lines in the bottom-right panel of Figure 5 (see Table 1 for a summary of all best-fit parameters). I08 fit a linear model to $v_{\phi}$ versus $Z$, but the difference between this result and their Equation (15) never exceeds $5 \mathrm{~km} \mathrm{~s}^{-1}$ for $Z<3 \mathrm{kpc}$. The errors on the power-law exponents of Equations (17) and (18) are $\sim 0.1$ and $\sim 0.2$, respectively.

However, a description of the velocity distribution based solely on the first and second moments (Equations (17) and (18)) does not fully capture the detailed behavior of our data. As already discussed by I08, the rotational-velocity distribution for disk stars is strongly non-Gaussian (see their Figure 13). It can be formally described by a sum of two Gaussians, with a fixed normalization ratio and a fixed offset of their mean values 

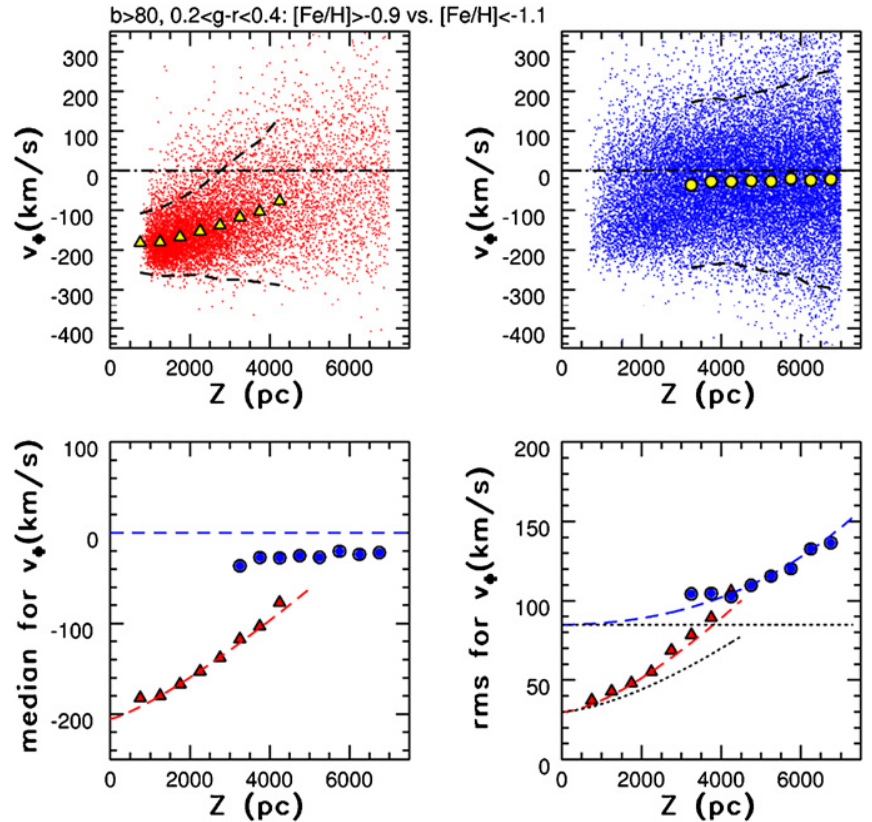

Figure 5. Dependence of the rotational velocity, $v_{\Phi}$, on distance from the Galactic plane for 14,000 high-metallicity $([\mathrm{Fe} / \mathrm{H}]>-0.9$; top-left panel) and 23,000 low-metallicity $\left([\mathrm{Fe} / \mathrm{H}]<-1.1\right.$, top right) stars with $b>80^{\circ}$. In the top two panels, individual stars are plotted as small dots, and the medians in bins of $Z$ are plotted as large symbols. The $2 \sigma$ envelope around the medians is shown by dashed lines. The bottom two panels compare the medians (left) and dispersions (right) for the two subsamples shown in the top panels and the dashed lines in the bottom two panels show predictions of a kinematic model described in the text. The dotted lines in the bottom-right panel show model dispersions without a correction for measurement errors (see Table 1).

(A color version of this figure is available in the online journal.)

for $|Z|<5 \mathrm{kpc}$,

$$
\begin{aligned}
p_{D}\left(x=v_{\phi} \mid Z\right)= & 0.75 G\left[x \mid v_{n}(Z), \sigma_{1}\right] \\
& +0.25 G\left[x \mid v_{n}(Z)-34 \mathrm{~km} \mathrm{~s}^{-1}, \sigma_{2}\right],
\end{aligned}
$$

where

$$
v_{n}(Z)=-194+19.2\left|\frac{Z}{\mathrm{kpc}}\right|^{1.25} \mathrm{~km} \mathrm{~s}^{-1}
$$

The intrinsic velocity dispersions, $\sigma_{1}$ and $\sigma_{2}$, are modeled as $a+b|Z|^{c}$, with best-fit parameters listed in Table 1 (see $\sigma_{\phi}^{1}$ and $\left.\sigma_{\phi}^{2}\right)$. Closer to the plane, in the $0.1<Z<2 \mathrm{kpc}$ range probed by red stars, the median rotational velocity and velocity dispersion are consistent with the extrapolation of fits derived here using much more luminous blue stars.

Figure 6 shows the $v_{\phi}$ distribution for four bins in $Z$ (analogous to Figure 13 from I08), overlaying two-component Gaussian fits with the measurement errors and $v_{n}(Z)$ as free parameters. The mean velocity and velocity dispersion exhibit $\sim 10 \mathrm{~km} \mathrm{~s}^{-1}$ variations relative to their expected values; while such deviations could be evidence of kinematic substructure, they are also consistent with the plausible systematic errors. We conclude that Equations (19) and (20) provide a good description of the disk kinematics for stars observed toward the North Galactic Pole, within the limitations set by the random and systematic errors in our data set.

The fits to the observed velocity distributions for halo and disk stars are shown in Figure 6 and demonstrate that the vertical gradients in median rotational velocity and velocity dispersion
Table 1

Best-fit Parameters $^{\mathrm{a}}$ for the Disk Velocity Distribution ${ }^{\mathrm{b}}$

\begin{tabular}{lccc}
\hline \hline Quantity & $a$ & $b$ & $c$ \\
\hline$\overline{v_{\phi}}{ }^{1}$ & -194 & 19.2 & 1.25 \\
$\sigma_{\phi}^{1}$ & 12 & 1.8 & 2.0 \\
$\sigma_{\phi}^{2}$ & 34 & 1.2 & 2.0 \\
$\sigma_{\phi}^{D}$ & 30 & 3.0 & 2.0 \\
$\sigma_{R}$ & 40 & 5.0 & 1.5 \\
$\sigma_{Z}$ & 25 & 4.0 & 1.5 \\
\hline
\end{tabular}

Notes. The uncertainties are typically $\sim 10 \mathrm{~km} \mathrm{~s}^{-1}$ for $a$, $\sim 20 \%$ for $b$ and $0.1-0.2$ for $c$.

${ }^{a}$ All listed quantities are modeled as $a+b|Z|^{c}$, with $Z$ in $\mathrm{kpc}$, and velocities in $\mathrm{km} \mathrm{s}^{-1}$.

$\mathrm{b}$ The $v_{\phi}$ distribution is non-Gaussian, and can be formally described by a sum of two Gaussians with a fixed normalization ratio $f_{k}: 1$, with $f_{k}=3.0$. The mean value for the second Gaussian has a fixed offset from the first Gaussian, $\overline{v_{\phi}}=\left(\overline{v_{\phi}}-\Delta \overline{v_{\phi}}\right)$, with $\Delta \overline{v_{\phi}}=34 \mathrm{~km} \mathrm{~s}^{-1}$. Extrapolation beyond $Z>5 \mathrm{kpc}$ is not reliable. The velocity dispersion for the second Gaussian is given by $\sigma_{\phi}^{2}$. If this non-Gaussianity is ignored, the $v_{\phi}$ dispersion is given by $\sigma_{\phi}^{D}$.

for disk stars seen in Figure 5 are not due to contamination by halo stars. To quantitatively assess the impact of "population mixing" as a result of the adopted metallicity-based classification $([\mathrm{Fe} / \mathrm{H}]<-1.1$ for "halo" stars and $[\mathrm{Fe} / \mathrm{H}]>-0.9$ for "disk" stars) on our measurements of these gradients, we have performed a series of Monte Carlo simulations. Assuming that the fits shown in Figure 6 accurately depict the intrinsic velocity distributions, and adopting analogous fits for their metallicity distributions (I08, their Figure 7), we have estimated the expected bias in median velocity and velocity dispersion for each population as a function of their relative normalization. As shown in Figure 6 from I08, the fraction of halo stars increases with distance from the plane, from about 0.1 at $Z=1 \mathrm{kpc}$ to about 0.9 at $Z=5 \mathrm{kpc}$. We find that the median rotation velocity and velocity dispersion biases are $<10 \mathrm{~km} \mathrm{~s}^{-1}$ for disk stars at $Z<3 \mathrm{kpc}$, as well as for halo stars at $Z>3.5 \mathrm{kpc}$. Furthermore, the biases are $<20 \mathrm{~km} \mathrm{~s}^{-1}$ for $Z<4 \mathrm{kpc}$ for disk stars and to $Z>3 \mathrm{kpc}$ for halo stars. At $Z=3 \mathrm{kpc}$, the contamination of both disk and halo subsamples by the other population is typically $\sim 10 \%-15 \%$. This small contamination and the use of the median (as opposed to the mean) and dispersion computed from the interquartile range results are reasonably small biases. With the adopted metallicity cutoffs, the sample contamination reaches $50 \%$ at $Z=1-1.5 \mathrm{kpc}$ for halo stars and at $Z=4.5 \mathrm{kpc}$ for disk stars.

The dependence of the Galactocentric radial velocity on $Z$ is shown for halo and disk subsamples in Figure 7. The median values (bottom-left panel) are consistent with zero, within the plausible systematic errors $\left(10-20 \mathrm{~km} \mathrm{~s}^{-1}\right)$, at all $Z$. The intrinsic dispersion for halo stars is consistent with a constant value of $\sigma_{R}^{H}=135 \pm 5 \mathrm{~km} \mathrm{~s}^{-1}$. For disk stars, the best-fit functional form $\sigma=a+b|Z|^{c}$ is

$$
\sigma_{R}^{D}=40+5\left|\frac{Z}{\mathrm{kpc}}\right|^{1.5} \mathrm{~km} \mathrm{~s}^{-1} .
$$

The $\sigma_{R}^{D} / \sigma_{\phi}^{D}$ ratio has a constant value of $\sim 1.35$ for $Z<1.5 \mathrm{kpc}$, and decreases steadily at larger $Z$ to $\sim 1$ at $Z \sim 4 \mathrm{kpc}$. 

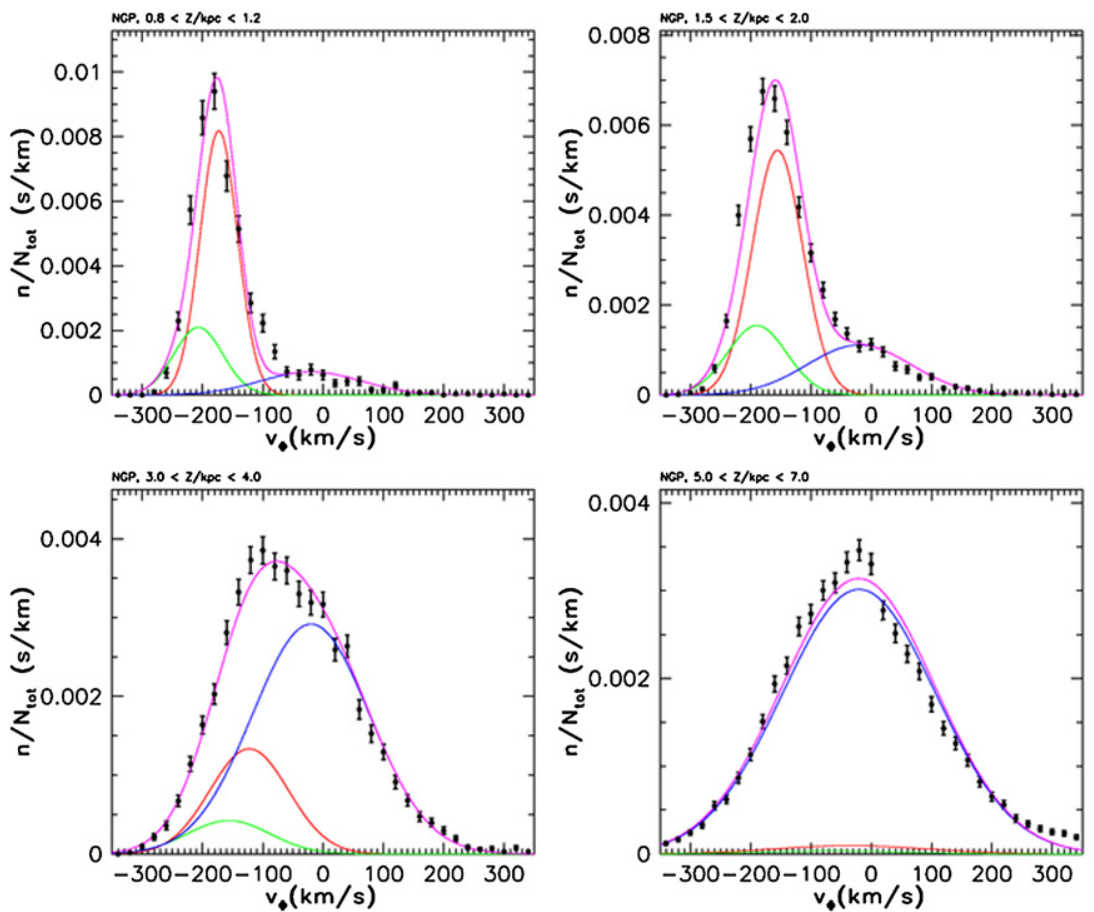

Figure 6. Symbols with error bars are the measured rotational-velocity distribution, $v_{\Phi}$, for stars with $0.2<g-r<0.4, b>80^{\circ}$, and $Z=0.8-1.2 \mathrm{kpc}$ (top left, $\sim 1500$ stars), $1.5-2.0 \mathrm{kpc}$ (top right, $\sim 4100 \mathrm{stars}$ ), $3.0-4.0 \mathrm{kpc}$ (bottom left, $\sim 6400$ stars) and 5.0-7.0 kpc (bottom right, $\sim 12,500$ stars). The red and green curves show the contribution of a two-component disk model (see Equations (19) and (20)), the blue curves show the Gaussian halo contribution, and the magenta curves are their sum. Note the difference in the scale of the $y$-axis between the top two panels.

(A color version of this figure is available in the online journal.)
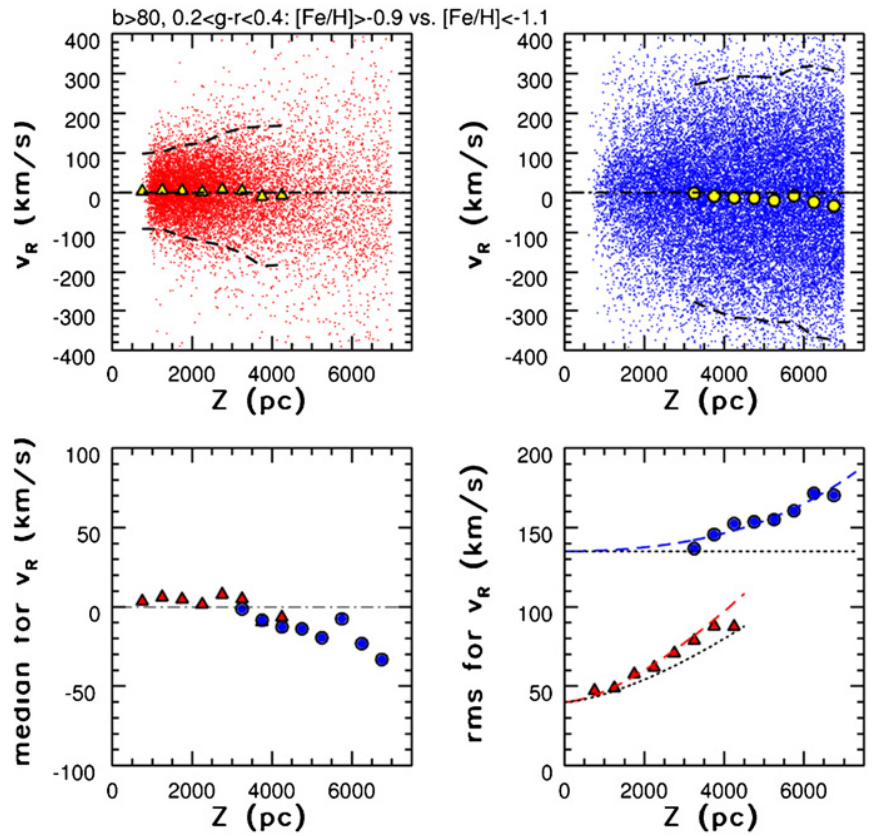

Figure 7. Analogous to Figure 5, but for the radial-velocity component, $v_{R}$. (A color version of this figure is available in the online journal.)

\subsection{Kinematics in the Meridional $Y \sim 0$ Plane}

The analysis of the rotational-velocity component can be extended to the meridional plane defined by $Y=0$, for which the longitudinal proper motion depends only on the rotationalvelocity component and the latitudinal proper motion, $v_{b}$, is a linear combination of radial and vertical components,

$$
v_{b}=\sin (b) v_{R}+\cos (b) v_{Z}
$$

Figure 8 plots $v_{\phi}$ and $v_{b}$ as functions of $R$ and $Z$ for halo and disk stars within $10^{\circ}$ of the meridional plane. The median $v_{b}$ is close to zero throughout most of the plotted region, as would be expected if the median $v_{R}$ and $v_{Z}$ are zero (the behavior of $v_{Z}$ is discussed in the next section). One exception is a narrow feature with $v_{b} \sim-100 \mathrm{~km} \mathrm{~s}^{-1}$ for $R<4 \mathrm{kpc}$. While a cold stellar stream could produce such a signature, its narrow geometry points directly at the observer. This behavior is also consistent with a localized systematic proper-motion error. Indeed, the bottom-left panel in Figure 1 shows that the systematic latitudinal proper-motion error at $l \sim 0^{\circ}, b \sim 45^{\circ}$

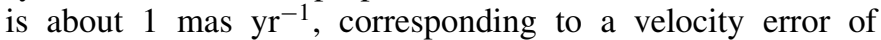
$\sim 100 \mathrm{~km} \mathrm{~s}^{-1}$ at a distance of $7 \mathrm{kpc}$.

As seen in the upper left panel of Figure 8, the median $v_{\phi}$ for halo stars is close to zero for $R<12 \mathrm{kpc}$. In the region with $R>12 \mathrm{kpc}$ and $Z<6 \mathrm{kpc}$, the median indicates a surprising prograde rotation in excess of $100 \mathrm{~km} \mathrm{~s}^{-1}$. This behavior is also seen in disk stars, and is likely due to the Monoceros stream, which has a metallicity intermediate between disk and halo stars and rotates faster than disk stars (see Sections 3.5.1 and 3.5.2 in I08). There is also an indication of localized retrograde rotation for halo stars with $Z \sim 9 \mathrm{kpc}$ and $R \sim 15 \mathrm{kpc}$ (corresponding to $l \sim 180^{\circ}, b \sim 50^{\circ}$, and a distance of $\sim 11 \mathrm{kpc}$ ). Stars with $Z=8-10 \mathrm{kpc}$ and $R=15-17 \mathrm{kpc}$ have median $v_{\phi}$ larger by $40 \mathrm{~km} \mathrm{~s}^{-1}(\mathrm{a} \sim 1 \sigma$ effect $)$ and median $[\mathrm{Fe} / \mathrm{H}]$ larger by 0.1 dex ( $\sim 5 \sigma$ effect) than stars with $Z=8-10 \mathrm{kpc}$ and $R=7-13 \mathrm{kpc}$. A systematic error in $\mu_{l}$ of $\sim 0.8$ mas yr$^{-1}$ is required to explain this kinematic feature as a data problem (although this would not explain the metallicity offset). However, the top-right panel in Figure 1 shows that the systematic $\mu_{l}$ errors in this sky region are below $0.5 \mathrm{mas} \mathrm{yr}^{-1}$, so this feature may well be real. We note that in roughly the same sky region and at roughly the same distance, Grillmair \& Dionatos (2006) have detected a narrow stellar stream. 

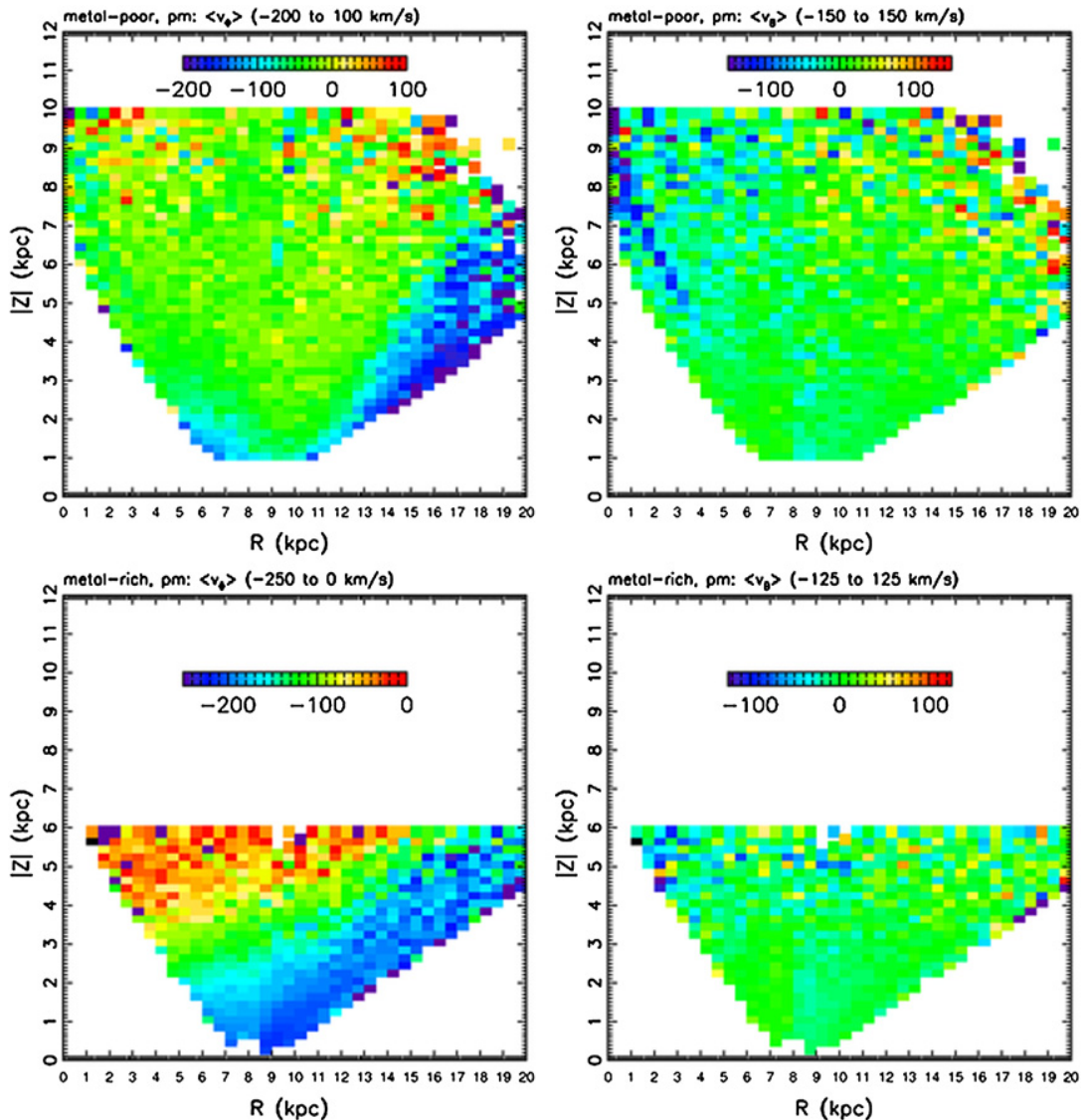

Figure 8. Dependence of velocity, measured using proper motions, on cylindrical Galactocentric coordinates for 172,000 metal-poor halo stars $([\mathrm{Fe} / \mathrm{H}]<-1.1$; top panels) and 205,000 metal-rich disk-like stars $\left([\mathrm{Fe} / \mathrm{H}]>-0.9\right.$; bottom panels). Stars are selected from three regions: $b>80^{\circ}$ (the North Galactic pole), $170^{\circ}<l<190^{\circ}$ (anticenter), and 350 $<l<10^{\circ}$ (Galactic center). The left column plots rotational velocity, $v_{\Phi}$, while the right column plots $v_{B}=\sin (b) v_{R}+\cos (b) v_{Z}$. To aid visualization of these boundaries, see the thick line in the top-left panel in Figure 1 . The median values of velocity in each bin are color-coded according to the legend shown in each panel. The measurements are reliable for distances up to about $7 \mathrm{kpc}$, but regions beyond this limit are shown for halo stars for completeness. The fraction of disk stars is negligible at such distances; their velocity distribution is shown for $Z<6 \mathrm{kpc}$. The region with negative velocity on the right side of top-left panel is due to contamination of the halo sample by stars from the Monoceros stream. The thin region with negative velocity on the left side of top-right panel is a data artifact (see the text).

In order to visualize the extent of "contamination" by the Monoceros stream, we replace the rotational velocity for each disk star by a simulated value drawn from the distribution described by Equation (19). We subtract this model from the data, and the residuals are shown in the right-hand panel of Figure 9. The position of the largest deviation is in excellent agreement with the position of Monoceros stream quantified in I08 ( $R=15-16 \mathrm{kpc}$ and $|Z| \sim 3-5 \mathrm{kpc})$. Further evidence for the presence of the Monoceros stream is shown in Figure 10, in which we analyze $v_{\phi}$ versus $[\mathrm{Fe} / \mathrm{H}]$, as a function of $R$, for blue stars at $Z=4-6$ ) $\mathrm{kpc}$. As is evident in the bottom-right panel, there is a significant excess of stars at $R>17 \mathrm{kpc}$ with $-1.5<[\mathrm{Fe} / \mathrm{H}]<-0.5$ that rotate in a prograde direction with $\sim 200 \mathrm{~km} \mathrm{~s}^{-1}$.

\section{ANALYSIS OF THE SPECTROSCOPIC SAMPLE}

Despite its smaller size, the SDSS DR7 spectroscopic sample of $\sim 100,000$ main-sequence stars is invaluable, because it enables a direct ${ }^{29}$ study of the three-dimensional velocity distribution. The sample extends to a distance of $\simeq 10 \mathrm{kpc}$, at which it can deliver velocity errors as small $\sim 10 \mathrm{~km} \mathrm{~s}^{-1}$

\footnotetext{
29 Statistical deprojection methods, such as that recently applied to a subsample of M stars discussed by Fuchs et al. (2009), can be used to indirectly infer the three-dimensional kinematics from proper-motion data.
}

(corresponding tangential-velocity errors are $\sim 150 \mathrm{~km} \mathrm{~s}^{-1}$ at a distance of $10 \mathrm{kpc}$ ). For each object in the SDSS spectroscopic survey, its spectral type, radial velocity, and radial-velocity error are determined by matching the measured spectrum to a set of stellar templates, which were calibrated using the ELODIE stellar library (Prugniel et al. 2007). Random errors on the radialvelocity measurements are a strong function of spectral type and signal-to-noise ratio, but are usually $<5 \mathrm{~km} \mathrm{~s}^{-1}$ for stars brighter than $g \sim 18$, rising sharply to $\sim 15 \mathrm{~km} \mathrm{~s}^{-1}$ for stars with $r=20$. We model the behavior of the radial-velocity errors as

$$
\sigma_{\mathrm{rad}}=3+12 \times 10^{0.4(r-20)} \mathrm{km} \mathrm{s}^{-1}
$$

We begin our analysis with blue disk and halo stars, and then briefly discuss the kinematics of nearby red M stars.

\subsection{Velocity Distributions}

We select 111,000 stars with $0.2<g-r<0.6(74,000$ have $b>20^{\circ}$ ) and, using their spectroscopic metallicity, separate them into 47,000 candidate halo stars with $[\mathrm{Fe} / \mathrm{H}]<-1.1$, and $\sim 53,000$ disk stars with $[\mathrm{Fe} / \mathrm{H}]>-0.9$. Assuming the spectroscopic metallicities accurately separate disk from halo stars, the use of photometric metallicity for the same selection would result in a contamination rate of $6 \%$ for the halo subsample, and $12 \%$ for the disk subsample. 

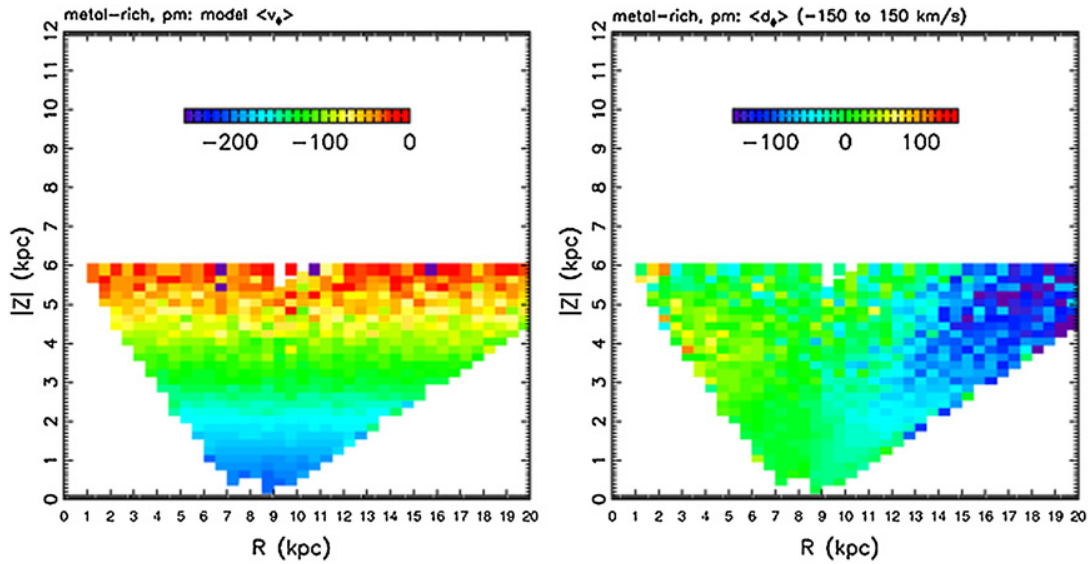

Figure 9. Left panel is analogous to the bottom-left panel in Figure 8, but for the model described in the text. The right panel shows the median difference between the data and model. Large discrepancies at $R>12 \mathrm{kpc}$ are due to the Monoceros stream (at $R=18 \mathrm{kpc}$ and $Z=4 \mathrm{kpc}$; disk stars rotate with a median $v_{\phi} \sim-100 \mathrm{~km} \mathrm{~s}{ }^{-1}$, while for the Monoceros stream, $v_{\phi} \sim-200 \mathrm{~km} \mathrm{~s}^{-1}$ ).
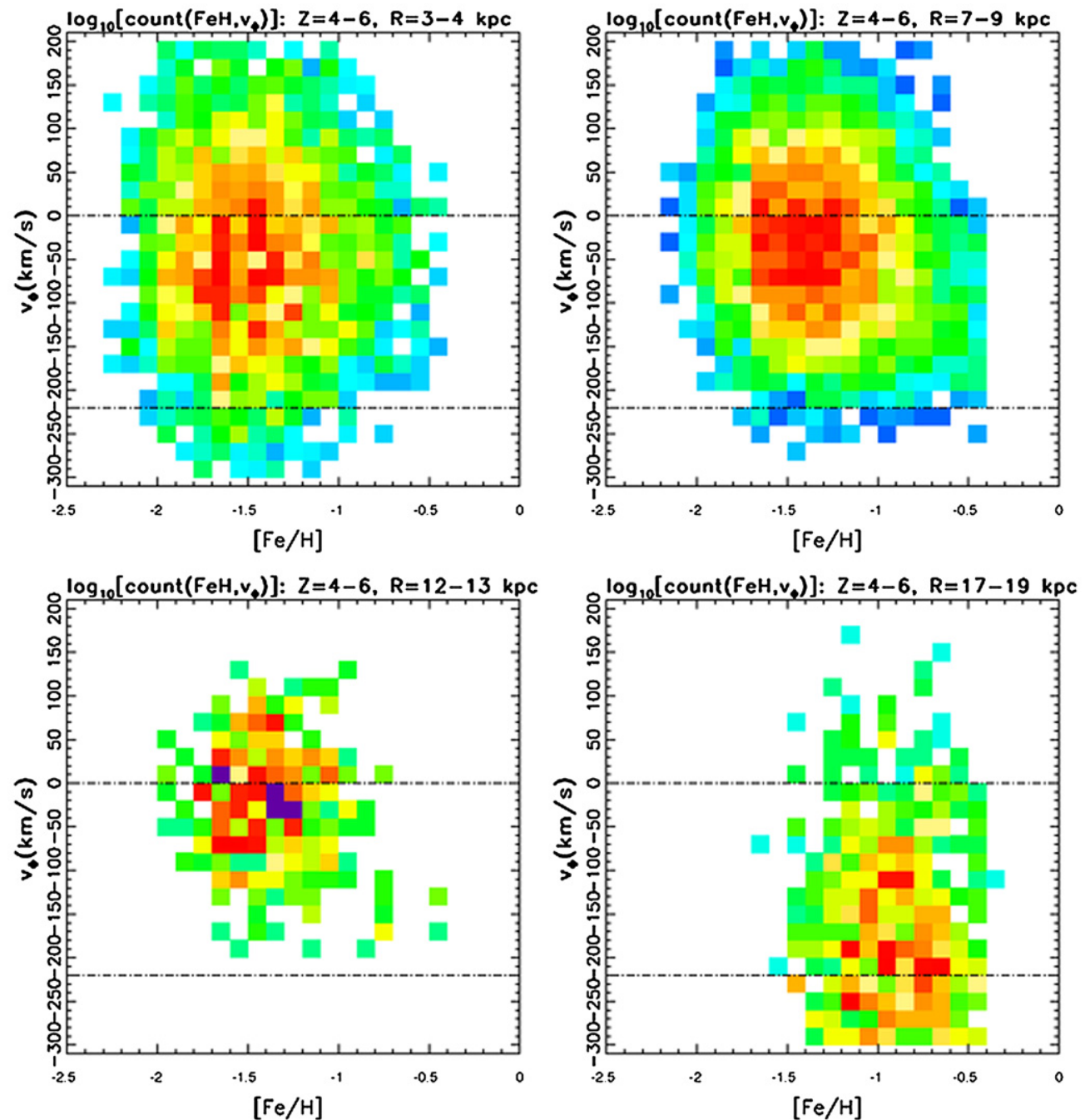

Figure 10. Distribution of stars with $0.2<g-r<0.4$ and $Z=4-6 \mathrm{kpc}$ in the rotational velocity vs. metallicity plane, for four ranges of Galactocentric cylindrical radius, $R$ (top left: 3-4 kpc; top right: 7-9 kpc; bottom left: 12-13 kpc; bottom right: 17-19 kpc). In each panel, the color-coded map shows the logarithm of counts in each pixel, scaled by the total number of stars. The horizontal lines at $v_{\Phi}=0 \mathrm{~km} \mathrm{~s}^{-1}$ and $v_{\Phi}=-220 \mathrm{~km} \mathrm{~s}^{-1}$ are added to guide the eye. High-metallicity $([\mathrm{Fe} / \mathrm{H}] \sim-1)$ stars with fast rotation $\left(v_{\Phi} \sim-220 \mathrm{~km} \mathrm{~s}^{-1}\right)$ visible in the bottom-right panel belong to the Monoceros stream, and are responsible for the features seen at $R>15 \mathrm{kpc}$ in the two left panels in Figure 8. 

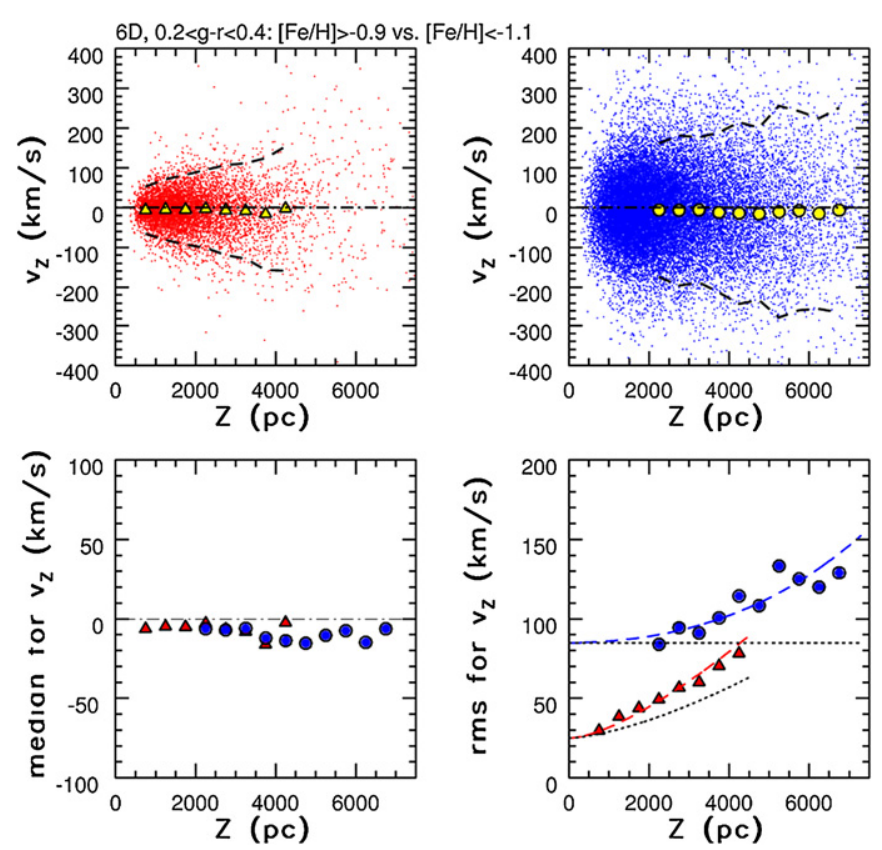

Figure 11. Similar to Figure 5, but for the vertical-velocity component, $v_{Z}$, and using a sample of stars with SDSS radial-velocity measurements, $0.2<g-r<0.4$ and $b>20^{\circ}(12,000$ stars in the high-metallicity subsample, and 38,000 stars in the low-metallicity subsample). An analogous figure for extended samples of 53,000 disk stars and 47,000 halo stars with $0.2<g-r<0.6$ has a similar appearance. The behavior of the rotationaland radial-velocity components in this sample is consistent with that shown in Figures 5 and 7.

(A color version of this figure is available in the online journal.)

The dependence of the median vertical velocity, $v_{z}$, and its dispersion on height above the disk, is shown in Figure 11 for the halo and disk subsamples. The median values of $v_{Z}$ are consistent with zero to better than $10 \mathrm{~km} \mathrm{~s}^{-1}$ at $Z<5 \mathrm{kpc}$, where statistical fluctuations are small.

As with $\sigma_{\phi}$ and $\sigma_{R}$, the vertical velocity dispersion can be modeled using a constant dispersion for halo stars $\left(\sigma_{Z}^{H}=\right.$ $85 \mathrm{~km} \mathrm{~s}^{-1}$ ), while for disk stars, the best-fit functional form is

$$
\sigma_{Z}^{D}=25+4\left|\frac{Z}{\mathrm{kpc}}\right|^{1.5} \mathrm{~km} \mathrm{~s}^{-1}
$$

The other two velocity components behave in a manner consistent with Equations (17), (18), and (21), just as they did in the proper-motion sample. This is encouraging, because the spectroscopic sample is collected over the entire northern hemisphere, unlike the proper-motion subsample studied in Section 3.1, which is limited to $b>80^{\circ}$.

The availability of all three velocity components in the spectroscopic sample makes it possible to study the orientation of the halo velocity ellipsoid. Figure 12 shows two-dimensional projections of the velocity distribution for subsamples of candidate halo stars with $0.2<g-r<0.4$. The top row corresponds to stars above the Galactic plane at $3<Z / \mathrm{kpc}<4$, while the bottom row is for stars the same distance below the plane. The velocity ellipsoid is clearly tilted in the top- and bottom-left panels, with a tilt angle consistent with $\tan ^{-1}\left(v_{Z} / v_{R}\right)=R / z$. While the tilt-angle errors are too large to obtain an improvement over existing measurements of $R_{\odot}$, it is remarkable that the northern and southern subsamples agree so well. ${ }^{30}$ In addition, when the $Z=3-5 \mathrm{kpc}$ sample is divided into three subsamples with $7<R / \mathrm{kpc}<11$, the tilt angle varies by the expected $\sim 8^{\circ}$ in the correct direction (see Figure 13). For all bins in the $R-Z$ plane, the best-fit tilt angle is statistically consistent (within $5^{\circ}$ ) with $\tan ^{-1}\left(v_{Z} / v_{R}\right)=R / z$. The other two projections of the velocity distribution for halo stars do not exhibit significant tilts to within $\sim 3^{\circ}$.

If we transform the velocities to a spherical coordinate system,

$$
\begin{aligned}
& v_{r}=v_{R} \frac{R}{R_{\mathrm{gc}}}+v_{Z} \frac{Z}{R_{\mathrm{gc}}} \\
& v_{\theta}=v_{R} \frac{Z}{R_{\mathrm{gc}}}-v_{Z} \frac{R}{R_{\mathrm{gc}}},
\end{aligned}
$$

where $r=R_{\mathrm{gc}}=\left(R^{2}+Z^{2}\right)^{1 / 2}$ is the spherical Galactocentric radius, we find no statistically significant tilt in any of the twodimensional velocity-space projections for halo stars (with tiltangle errors ranging from $\sim 1^{\circ}$ to $\sim 5^{\circ}$ ).

As shown in Figure 14, we see no evidence for a velocityellipsoid tilt in $v_{Z}$ versus $v_{R}$ for the disk stars. The plotted subsamples are again selected to have colors $0.2<g-r<0.4$, but are selected closer to the Galactic plane, $|Z|=1.5-2.5 \mathrm{kpc}$, in order to improve statistics and reduce contamination from halo stars. The velocity-ellipsoid tilt is consistent with zero within $\sim 1 \sigma$, and alignment of the velocity ellipsoid with the spherical coordinate system of Equation (25) is ruled out at a $\sim 2 \sigma$ or greater confidence level for each of five analyzed $R-Z$ bins $(R=6-11 \mathrm{kpc}$, with $\Delta R=1 \mathrm{kpc})$. We conclude that there is no evidence for a velocity-ellipsoid tilt in the disk subsample, but caution that, due to the small $Z$ range, the data cannot easily distinguish between cylindrical and spherical alignment. A model prediction for velocity-ellipsoid tilt is discussed below.

The vertex deviation is analogous to the velocity-ellipsoid tilt discussed above, but is defined in the $v_{\phi}$ versus $v_{R}$ plane instead of the $v_{Z}$ versus $v_{R}$ plane. The same plots for red $(g-r>0.6$, median 1.2) disk stars are shown in the center top and bottom panels in Figure 15. These stars can be traced closer to the plane, $|Z|=0.6-0.8 \mathrm{kpc}$; in both hemispheres, the data are consistent with a vertex deviation of $\sim 20^{\circ}$, with an uncertainty of $\sim 10^{\circ}$. This result is consistent with the vertex deviation obtained by Fuchs et al. (2009).

Another interpretation for the $v_{\phi}$ versus $v_{R}$ distribution of disk stars invokes a two-component velocity distribution, which can result in a similar deviation even if each component is perfectly symmetric in the cylindrical coordinate system. A. Kowalski et al. (2010, in preparation) find that the $v_{\phi}$ versus $v_{R}$ distribution for red stars toward the North Galactic Pole, with $0.1<Z / \mathrm{kpc}<1.5$, can be fitted by a sum of two Gaussian distributions that are offset from each other by $\sim 10 \mathrm{~km} \mathrm{~s}^{-1}$ in each direction. This offset results in a nonzero vertex deviation if the sample is not large enough, or if measurements are not sufficiently accurate, to resolve the two Gaussian components. This double-Gaussian structure would be at odds with the classical description based on the Schwarzschild approximation-we refer the interested reader to the Kowalski et al. study for more details. Unfortunately, the spectroscopic samples are not large enough to distinguish a two-component model from the standard interpretation.

\footnotetext{
30 A plausible, if somewhat optimistic, tilt-angle uncertainty of $1^{\circ}$ corresponds to an $R_{\odot}$ error of $0.5 \mathrm{kpc}$; extending the sample to $|Z|=8 \mathrm{kpc}$ could deliver errors of $0.3 \mathrm{kpc}$ per bin of a similar size.
} 

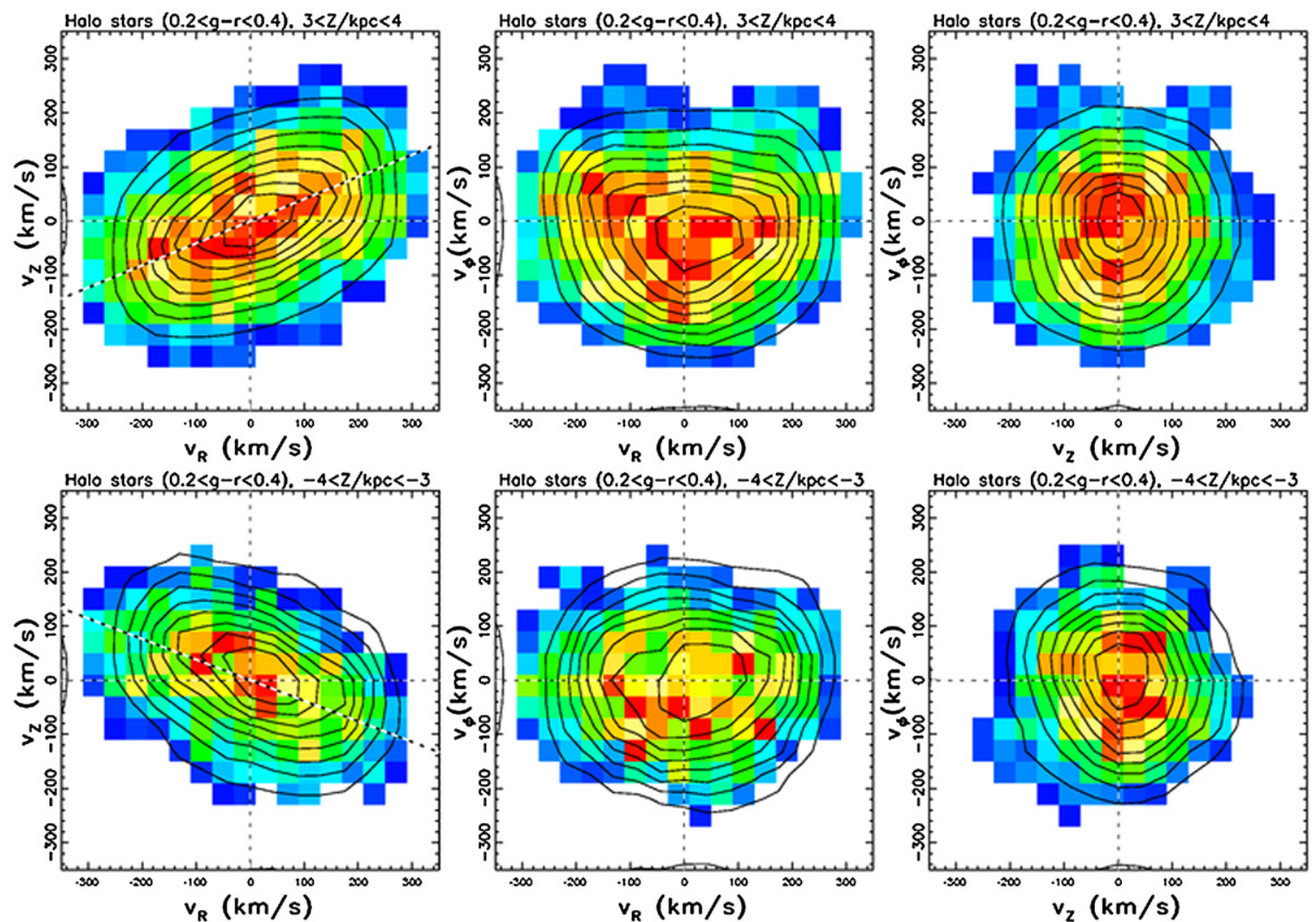

Figure 12. Three two-dimensional projections of the velocity distribution for two subsamples of candidate halo stars selected using spectroscopic metallicity $(-3<[\mathrm{Fe} / \mathrm{H}]<-1.1)$ and with $6<R / \mathrm{kpc}<11$. The top row corresponds to 2700 stars with distances, $3<Z / \mathrm{kpc}<4$, and the bottom row to 1300 stars with $-4<Z / \mathrm{kpc}<-3$. The distributions are shown using linearly spaced contours, and with a color-coded map showing smoothed counts in pixels (low to high from blue to red). The measurement errors are typically $60 \mathrm{~km} \mathrm{~s}^{-1}$. Note the strong evidence for a velocity-ellipsoid tilt in the top and bottom-left panels (see also Figure 13). The two dashed lines in these panels show the median direction toward the Galactic center.
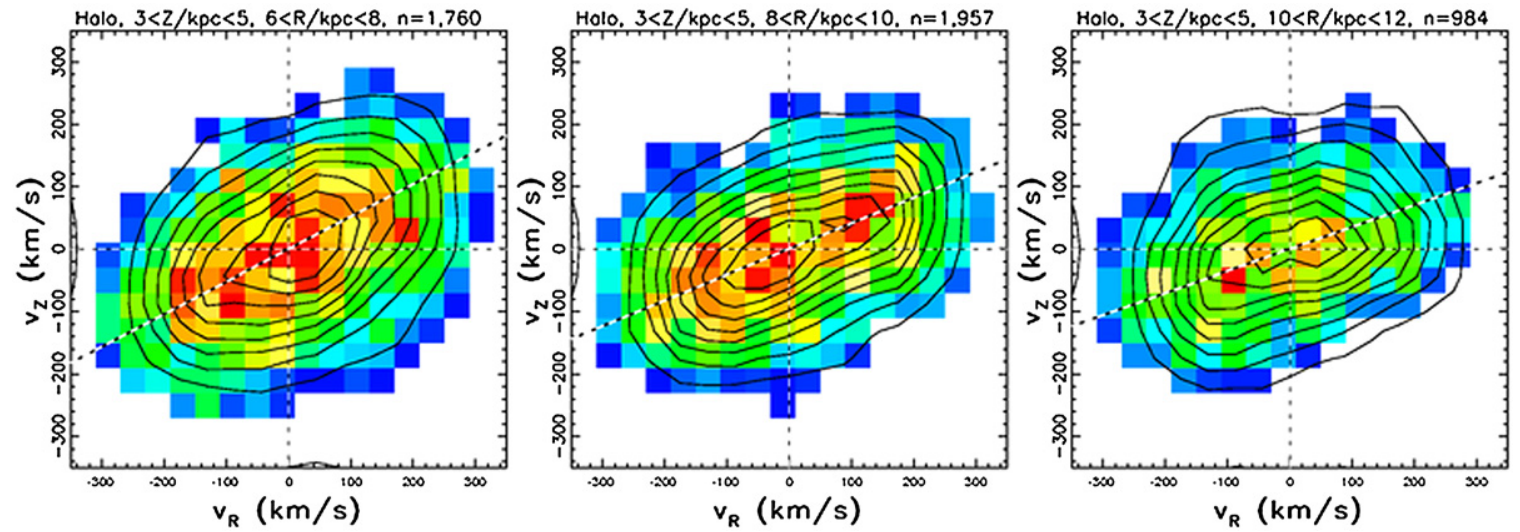

Figure 13. Illustration of the velocity-ellipsoid tilt-angle variation. Analogous to Figure 12, except that only the $v_{Z}$ vs. $v_{R}$ projection is shown for a constant $Z$, for three ranges of $R$, as marked on the top of each panel.

\subsubsection{A Model Prediction for Velocity-Ellipsoid Tilt}

The tilt of the velocity-ellipsoid tilt in the $v_{Z}-v_{R}$ plane is calculated using methods developed in Kent \& de Zeeuw (1991), who show that the tilt of the ellipsoid at any point in the Milky Way depends not only on the gravitational potential of the Galaxy but also on the isolating integrals of a particular orbit and thus (weakly) on the distribution function of stellar velocities. We use the model of the Milky Way gravitational field from Carlberg \& Innanen (1987), scaled to a solar radius of $8 \mathrm{kpc}$ and a circular velocity of $220 \mathrm{~km} \mathrm{~s}^{-1}$. At a particular point in the Galaxy, we integrate eight orbits, each one launched from that point, with velocities in each of the $R, Z$, and $\phi$ coordinates that are $\pm 1 \sigma$ from the systemic velocity. We use the observed velocity distributions to obtain the systemic velocity and the $1 \sigma$ values. Furthermore, using the "least-squares fitting" method, we determine the parameters of the prolate spheroidal coordinate system that best matches each orbit-from this, we can compute its contribution to the velocity ellipsoid. For some orbits (especially those of halo stars), the orbits are not local to 

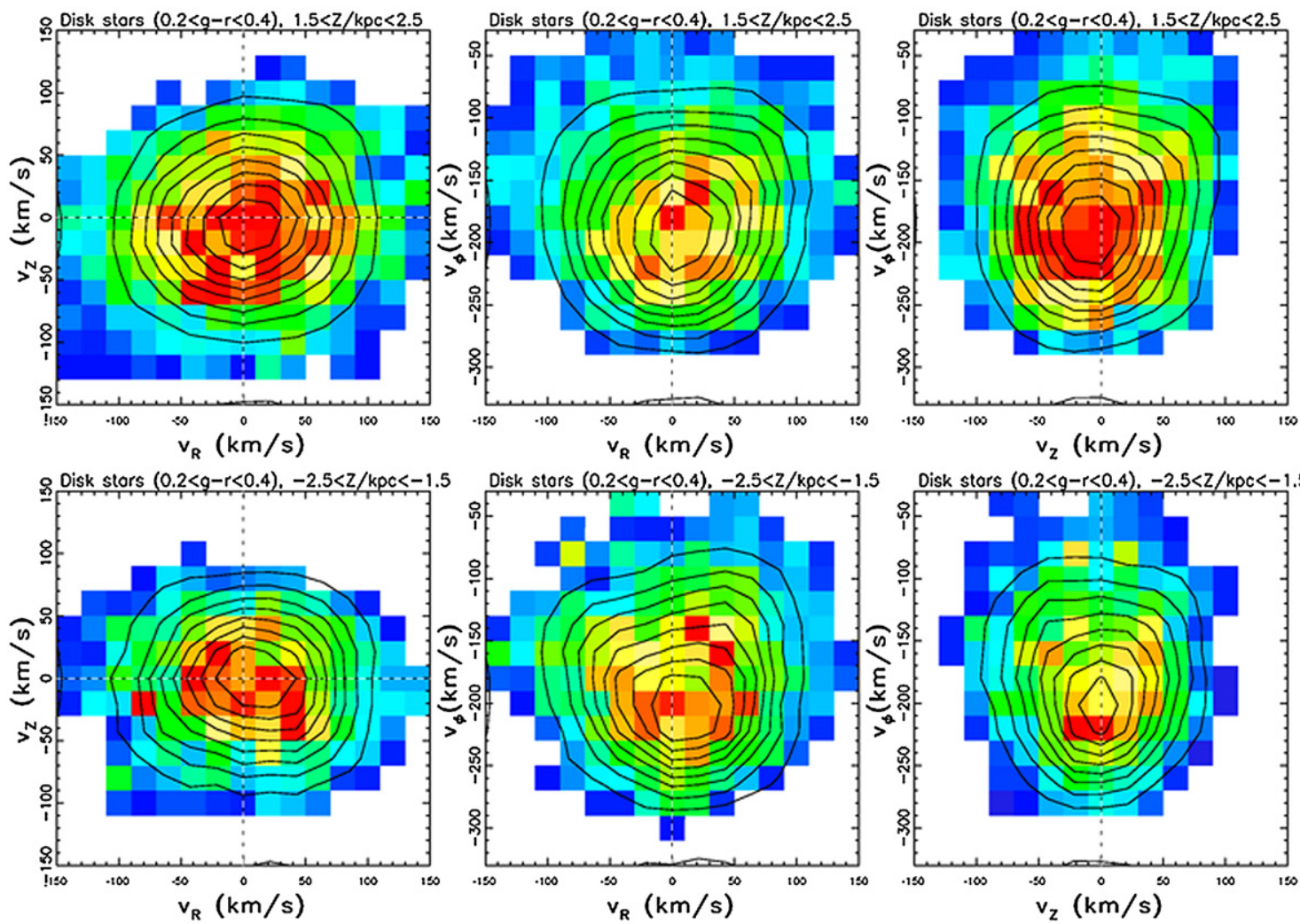

Figure 14. Analogous to Figure 12, except that the velocity distribution is shown for two subsamples of candidate disk stars selected using spectroscopic metallicity $([\mathrm{Fe} / \mathrm{H}]>-0.9)$. The top row corresponds to 2200 stars with distances from the Galactic plane $1.5<Z / \mathrm{kpc}<2.5$, and the bottom row to 1500 stars with $-2.5<Z / \mathrm{kpc}<-1.5$. The measurement errors are typically $35 \mathrm{~km} \mathrm{~s}^{-1}$. Note the absence of velocity-ellipsoid tilt in the top and bottom-left panels.

(A color version of this figure is available in the online journal.)
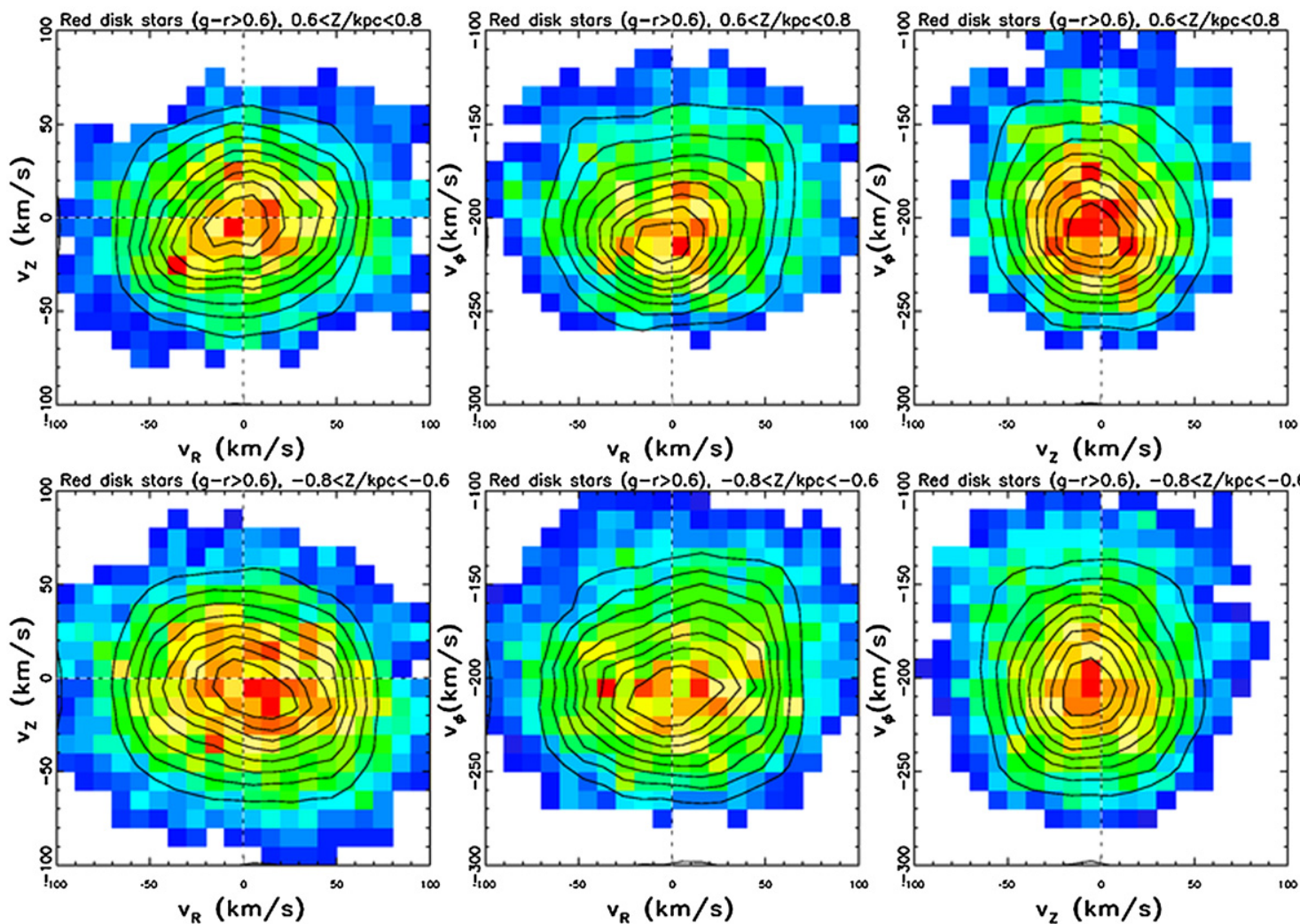

Figure 15. Analogous to Figure 14, except that the velocity distribution is shown for two subsamples of red stars $(g-r>0.6)$ : the top row corresponds to 3000 stars with distances from the Galactic plane $0.6<Z / \mathrm{kpc}<0.8$, and the bottom row to 4600 stars with $-0.8<Z / \mathrm{kpc}<-0.6$. The measurement errors are typically $\sim 15 \mathrm{~km} \mathrm{~s}^{-1}$. There is no strong evidence in these panels for a tilt in the velocity ellipsoid.

(A color version of this figure is available in the online journal.) 
Table 2

A Model Prediction for Velocity-Ellipsoid Tilt ${ }^{\mathrm{a}}$

\begin{tabular}{lccc}
\hline \hline$R(\mathrm{kpc})$ & $Z(\mathrm{kpc})$ & $\theta_{R Z}(\mathrm{deg})$ & $\arctan (Z / R)(\mathrm{deg})$ \\
\hline 8.0 & 0.7 & 3 & 5.0 \\
8.0 & 2.0 & 11 & 14.0 \\
8.0 & 3.5 & 26 & 23.6 \\
7.0 & 4.0 & 32 & 29.7 \\
9.0 & 4.0 & 25 & 24.0 \\
11.0 & 4.0 & 21 & 20.0 \\
\hline
\end{tabular}

Notes. ${ }^{\text {a }}$ The first two columns determine the position in the Galaxy. The third column lists the predicted orientation of the velocity ellipsoid computed as described in Section 4.1.1. The fourth column lists the orientation of a velocity ellipsoid pointed toward the Galactic center.

the solar neighborhood, and the local fitting method cannot fit the entire orbit. For these cases, we confine the orbits to radii greater than $4 \mathrm{kpc}$. Since the only purpose of the fit is to model the kinematics in a small volume of space, the fact that the fit is no longer global is of no consequence. The scatter in tilt angle among the individual orbits at each position depends only weakly on position $(\sim 10 \%)$.

Table 2 gives the predicted tilt angles for each position plotted in Figures 12-15. For the halo stars plotted in Figures 12 and 13, where the ellipsoid shows the most distinctive tilt, the predictions match the observed tilts quite well, but are slightly steeper at $Z>3 \mathrm{kpc}$ than the $Z / R$ relation predicted for spherical symmetry. For the disk stars in Figures 14 and 15, the predicted tilts lie between the cases of cylindrical and spherical symmetry. The observed ellipsoid is sufficiently round, however, that no definitive comparison can be made.

\subsection{Direct Determination of the Solar Peculiar Motion}

If there is no net streaming motion in the $Z$ direction in the solar neighborhood, the median heliocentric, $v_{Z}^{\text {obs }}$, for nearby stars should be equal to $v_{Z}^{\odot}\left(7 \mathrm{~km} \mathrm{~s}^{-1}\right.$, based on an analysis of HIPPARCOS results by Dehnen \& Binney 1998). We do not expect a large velocity gradient within $\sim 1 \mathrm{kpc}$ from the Sun, so we select from the spectroscopic sample $\sim 13,000 \mathrm{M}$ dwarfs with $2.3<g-i<2.8$. In the northern hemisphere, we have 5700 stars with a median heliocentric velocity, $\left\langle v_{Z}^{\text {obs }}\right\rangle=$ $-1.8 \mathrm{~km} \mathrm{~s}^{-1}$, while for stars in the southern hemisphere we obtain $\left\langle v_{Z}^{\mathrm{obs}}\right\rangle=-11.0 \mathrm{~km} \mathrm{~s}^{-1}$. This difference is likely due to a systematic radial-velocity error. If we simultaneously vary an assumed radial-velocity error, $\Delta_{\mathrm{rad}}$, and the solar peculiar motion, $v_{Z}^{\odot}$, while requiring that the median $v_{Z}^{\text {obs }}$ should be the same for both hemispheres, we obtain $\Delta_{\text {rad }}=5.0 \pm 0.4 \mathrm{~km} \mathrm{~s}^{-1}$ and $v_{Z}^{\odot}=6.5 \pm 0.4 \mathrm{~km} \mathrm{~s}^{-1}$. This value for $v_{Z}^{\odot}$ is in excellent agreement with the HIPPARCOS value of $7.2 \pm 0.4 \mathrm{~km} \mathrm{~s}^{-1}$ (Dehnen \& Binney 1998). This systematic offset in SDSS radial velocities is probably due to the small number of ELODIE templates for red stars. It is likely that the adoption of improved templates from Bochanski et al. (2007a) will yield smaller systematic errors. A similar analysis for blue stars does not yield a robust detection of the velocity offset. A detailed comparison of SDSS radial velocities with radial-velocity standards from the literature arrived at the same null result for blue stars.

As with the vertical component of the solar peculiar motion, if the adopted value of $v_{X}^{\odot}=-10 \mathrm{~km} \mathrm{~s}^{-1}$ were incorrect, the median $v_{R}$ would deviate from zero. The rms scatter of the median $v_{R}$ for subsamples of nearby $\mathrm{M}$ stars selected by distance and color is $0.5 \mathrm{~km} \mathrm{~s}^{-1}$, which is an upper limit on the error in the adopted value of $v_{X}^{\odot}$. This result, which is based on the full three-dimensional velocity distribution, agrees well with results from indirect statistical deprojection methods using only proper motions (Dehnen 1998; Fuchs et al. 2009).

For both blue and red disk stars, the extrapolation of the median $v_{\phi}$ to $Z=0$ yields $-205 \mathrm{~km} \mathrm{~s}^{-1}$. Since we corrected stellar velocities for an assumed solar motion of $-225 \mathrm{~km} \mathrm{~s}^{-1}$, this implies that the $Y$ component of the solar velocity relative to the bulk motion of stars in the solar neighborhood is $20 \mathrm{~km} \mathrm{~s}^{-1}$, in agreement with recent results obtained by Fuchs et al. (2009) for the same data set. A similar value was obtained by Dehnen \& Binney (1998) for their ${ }^{31}$ subsample of red stars within $100 \mathrm{pc}$.

\section{A MODEL FOR THE KINEMATICS OF DISK AND HALO STARS}

Informed by the results from the preceding two sections, we introduce a model that aims to describe the global behavior of the observed stellar kinematics. In our model, we do not attempt to account for kinematic substructure (e.g., the Monoceros stream), or the Galactic bulge region, nor do we incorporate any complex kinematic behavior close to the Galactic plane. Nevertheless, we attempt to capture the gross properties of the data in the volume probed by SDSS, including the bulk kinematic trends and the kinematic differences between high-metallicity disk stars and low-metallicity halo stars. We describe the model in Section 5.1, then test it in Section 5.2 using both the proper-motion and radial-velocity samples.

\subsection{The Kinematic Model}

For halo stars, a single velocity ellipsoid (expressed in a Galactocentric spherical coordinate system, see Section 4.1) is a good description of the gross halo kinematics within the $10 \mathrm{kpc}$ distance limit of our sample. Our model assumes that the halo has no net rotation (see below for a test of this assumption), and that the principal axes of the velocity ellipsoid are aligned with a spherical coordinate system. The velocity dispersions measured in Sections 3 and $4, \sigma_{R}^{H}=135 \mathrm{~km} \mathrm{~s}^{-1}, \sigma_{\phi}^{H}=85 \mathrm{~km} \mathrm{~s}^{-1}$, and $\sigma_{Z}^{H}=85 \mathrm{~km} \mathrm{~s}^{-1}$, are expressed in a cylindrical coordinate system. The interplay between proper-motion, radial-velocity, and distance measurement errors is complex, so we use Monte Carlo simulations to translate them to spherical coordinates; the results of this exercise are $\sigma_{r}^{H}=141 \mathrm{~km} \mathrm{~s}^{-1}$ and $\sigma_{\theta}^{H}=$ $75 \mathrm{~km} \mathrm{~s}^{-1}$ ( $\sigma_{\phi}^{H}$ is unchanged), with uncertainties of $\sim 5 \mathrm{~km} \mathrm{~s}^{-1}$.

For disk stars within $\sim 1-2 \mathrm{kpc}$ from the Sun, the velocitymeasurement errors are sufficiently small, and the samples are sufficiently large, to resolve rich kinematic substructure (e.g., Figure 3). This behavior is quantified in detail in A. Kowalski et al. (2010, in preparation), but here we simply use the twocomponent model given by Equations (19) and (20) to describe the non-Gaussian $v_{\phi}$ distribution and velocity shear seen for disk stars. Furthermore, we assume that $v_{R}$ and $v_{Z}$ have uncorrelated Gaussian distributions, with zero mean and the Z-dependent intrinsic dispersion parameters listed in Table 1. As discussed in Section 4, there is no compelling evidence for a tilt in the velocity ellipsoid of blue disk stars in the $v_{R}-v_{Z}$ plane, so we model the disk velocity ellipsoid in cylindrical coordinates.

\footnotetext{
31 They extrapolated the mean azimuthal motion of color-selected samples, which is correlated with the radial-velocity dispersion, to zero dispersion and obtained $v_{Y}^{\odot, \text { pec }}=-5.3 \mathrm{~km} \mathrm{~s}^{-1}$, used here.
} 

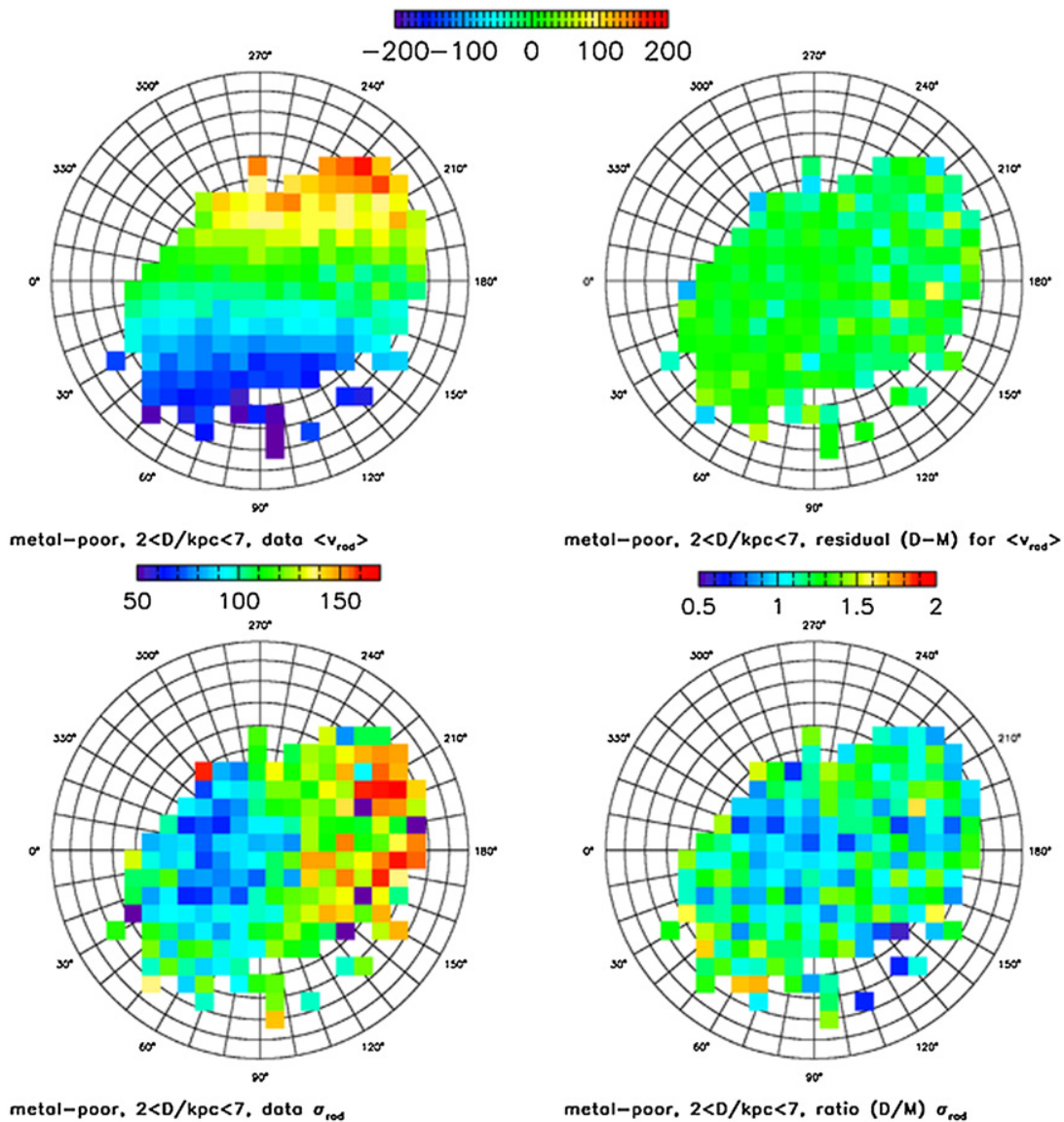

Figure 16. Comparison of medians and dispersions for the measured and modeled radial velocities of 20,000 blue $(0.2<g-r<0.4)$ halo stars (spectroscopic $[\mathrm{Fe} / \mathrm{H}]<-1.1)$ at distances, $D=2-7 \mathrm{kpc}$, and $b>20^{\circ}$. The top-left panel shows the median measured radial velocity in each pixel, color-coded according to the legend shown at the top (units are $\mathrm{km} \mathrm{s}^{-1}$ ). The top-right panel shows the difference between this map and an analogous map based on model-generated values of radial velocity, using the same scale as in the top-left panel. The bottom-left panel shows the dispersion of measured radial velocities, color-coded according to the legend above it. The bottom-right panel shows the ratio of this map and an analogous map based on model-generated values of radial velocity, color-coded according to the legend above it. When the sample is divided into $1 \mathrm{kpc}$ distance shells, the behavior is similar.

\subsection{Global Model Tests}

Our model predicts distributions of the three measured kinematic quantities, $v_{\mathrm{rad}}, \mu_{l}$, and $\mu_{b}$, for an arbitrary control volume defined by color, magnitude, and sky coordinates. We can test the consistency of this model, $m$, with our data, $d$, by computing the residuals of each kinematic quantity,

$$
\chi=\frac{d-m}{\left(\sigma_{d}^{2}+\sigma_{m}^{2}\right)^{1 / 2}},
$$

where $\sigma_{d}$ is the measurement error of the data and $\sigma_{m}$ is the dispersion predicted by the model. For all three kinematic quantities, we find $\bar{\chi} \simeq 0$ to within 0.05 for all three quantities, with dispersions of $\sim 1.05-1.1$. While this result is a necessary condition for the model to be acceptable, it is not sufficient. A stronger test of the model was shown in Figure 9 for disk stars, but in what follows, we perform additional tests that cover all sky regions with available data.

\subsubsection{Tests With the Radial-Velocity Sample}

Figure 16 compares the medians and dispersions for the measured and modeled radial velocities of halo stars. The alignment of the velocity ellipsoid with spherical Galactocentric radius is clearly seen in the bottom-left panel, where the data show a dispersion gradient moving away from the Galactic Center.
The increased dispersion toward $l=180^{\circ}$ was misinterpreted by Ivezić et al. (2006) to be a sign of substructure. There are no large discrepancies between the measured and predicted behavior - the median value of the difference between observed and modeled values is $4.6 \mathrm{~km} \mathrm{~s}^{-1}$, with a scatter of $19 \mathrm{~km} \mathrm{~s}^{-1}$ (see top-right panel). A similar scatter is obtained between two model realizations with the same number of stars and measurement errors. The dispersion ratio, $\sigma_{\text {rad }}^{d} / \sigma_{\text {rad }}^{m}$, is centered on 1.13 , with a scatter of 0.2 (see bottom-right panel). For pairs of model realizations, the ratio is always centered on one to within 0.02 , with a scatter of 0.2 , suggesting that the observed velocity dispersion is about $10 \%$ larger than predicted by our smooth model. If the residuals were due to halo rotation, we would expect a spatial coherence. Similarly, for disk stars at $1<d<2.5 \mathrm{kpc}$, the median radial-velocity residual is $2.8 \mathrm{~km} \mathrm{~s}^{-1}$, with a scatter of $6.6 \mathrm{~km} \mathrm{~s}^{-1}$ (not shown).

\subsubsection{Tests Based on the Proper-motion Sample}

The large size of the proper-motion sample enables a much higher spatial resolution when searching for structure in the model residuals. We have compared the observed and modeled proper-motion distributions in narrow bins of distance, across the sky, and separately for disk and halo subsamples. As an illustration, Figures 17 and 18 show the median longitudinal and latitudinal proper motion observed for disk stars. There is very little change in the proper-motion distribution among different 


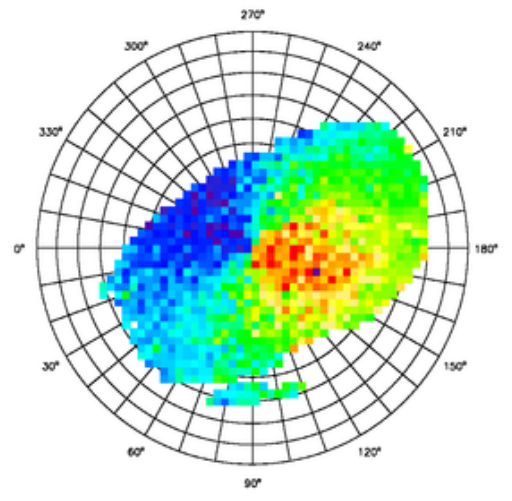

metal-rich, $0.8<\mathrm{D} / \mathrm{kpc}<1.2,<\mu_{\mathrm{l}}>$

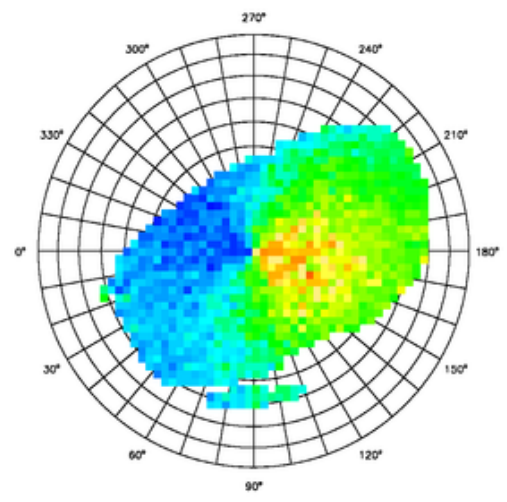

metol-rich, $1.8<\mathrm{D} / \mathrm{kpc}<2.2,\left\langle\mu_{\mathrm{l}}\right\rangle$

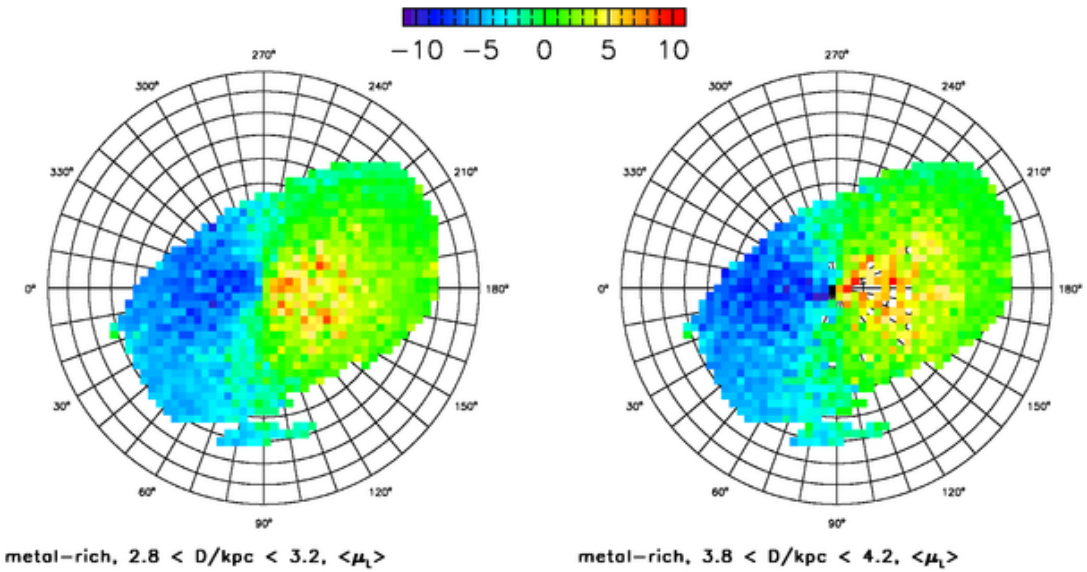

Figure 17. Distribution of the median longitudinal proper motion in a Lambert projection of the North Galactic cap for high-metallicity ([Fe/H] $>-0.9$ ), blue $(0.2<g-r<0.4)$ stars, in several distance bins (top left: 58,000 stars with $D=0.8-1.2 \mathrm{kpc}$; top right: 119,000 stars with $D=1.8-2.2 \mathrm{kpc}$; bottom left: 72,000 stars with $D=2.8-3.2 \mathrm{kpc}$; bottom right: 43,000 stars with $D=3.8-4.2 \mathrm{kpc}$ ). All maps are color-coded using the same scale, shown in the middle (units are mas $\mathrm{yr}^{-1}$ ). Note that the magnitude of the proper motion does not change appreciably as the distance varies from $\sim 2 \mathrm{kpc}$ to $\sim 4 \mathrm{kpc}$; this is due to a vertical gradient in the rotational velocity for disk stars (see Figure 3).

(A color version of this figure is available in the online journal.)

distance bins, due to the nearly linear vertical rotational-velocity gradient. The residuals for longitudinal proper motion are shown in Figure 19. They provide weak evidence for either substructure or a radial gradient that is not modeled, but it is difficult to distinguish between these two possibilities with these data alone.

A comparison of disk and halo subsamples in a distance bin centered on $d=4 \mathrm{kpc}$ is shown in Figure 20. The largest data versus model discrepancy for halo stars, seen in the bottom-left panel toward $l \sim 0^{\circ}$, is also seen from a different viewing angle in the top-left panel of Figure $8(R \sim 6 \mathrm{kpc}$ and $Z \sim 2 \mathrm{kpc})$. It is likely that this discrepancy is due to contamination of the halo sample by metal-poor disk stars. Figure 21 shows the residuals for halo stars selected from the $8-10 \mathrm{kpc}$ distance bin. The residuals for both proper-motion components exhibit similar morphology to the systematic proper-motion errors plotted in the two left panels of Figure 1. In this distance bin, they correspond to velocity errors of $\sim 30 \mathrm{~km} \mathrm{~s}^{-1}$; as such, kinematic substructure in the halo will be difficult to discern with this sample at $\gtrsim 10 \mathrm{kpc}$. We note that it is tempting to associate the coherent $\mu_{l}$ residuals toward $l \sim 300^{\circ}$ and $l \sim 60^{\circ}$ with the Virgo overdensity (see J08; An et al. 2009). However, the top-left panel in Figure 1 clearly shows systematic proper-motion errors of the required amplitude $\left(\sim 1.5 \mathrm{mas} \mathrm{yr}^{-1}\right)$ in the same sky region.

Schlaufman et al. (2009) use SDSS radial-velocity measurements for metal-poor turnoff stars to search for pieces of cold debris streams (which they term Elements of Cold Halo substruc- ture, or ECHOs). In 137 lines of sight they detect 10 ECHOs. The six northern ECHOs from their Table 2 (class I peak detections) are shown as white circles in the bottom two panels of Figure 21. It seems plausible that four of these ECHOs might be associated with the Monoceros stream $\left(b \sim 30^{\circ}\right)$, while one of the two remaining detections (at $l=162^{\circ} .4$ and $l=59^{\circ} .2$ ) is associated with the Grillmair \& Dionatos (2006) stream. The $\mathrm{ECHO}$ at $l=100.7$ and $b=56.8$ remains unassociated with any known substructure.

We conclude that our model reproduces the first and second moments of the velocity distributions reasonably well for both disk and halo stars. Except in the region close to the Monoceros stream, the non-Gaussian $v_{\phi}$ distribution for disk stars is also well-described. On average, the model agrees with the data to within $\sim 1 \mathrm{mas} \mathrm{yr}^{-1}$ for proper motions, and $\sim 10 \mathrm{~km} \mathrm{~s}^{-1}$ for radial velocities.

\subsection{Constraints on $v_{\mathrm{LSR}}$ from Large-scale Halo Kinematics}

The proper-motion distribution for halo stars toward the Galactic poles depends only on the difference between the velocity of the local standard of rest (given that the solar peculiar motion is known to $\sim 1 \mathrm{~km} \mathrm{~s}^{-1}$ ), $v_{\mathrm{LSR}}$, and $v_{\phi}$ for halo stars. At least in principle, samples that extend over a large sky area can be used to provide constraints on both the halo rotation and $v_{\text {LSR }}$. Figure 22 compares the radial velocity and longitudinal propermotion residuals between two models with $\left(v_{\phi}^{\text {halo }}, v_{\text {LSR }}\right)=$ 


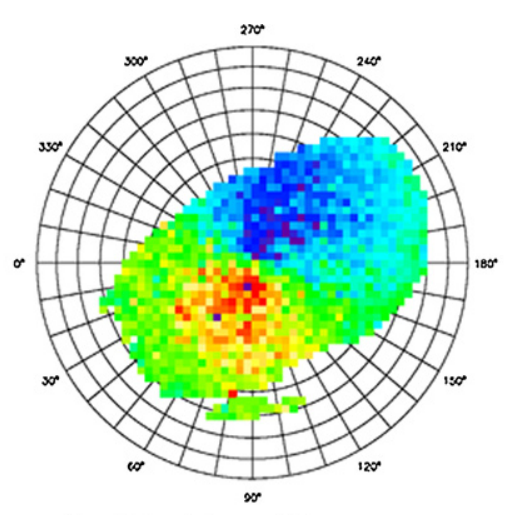

metal-rich, $0.8<0 / \mathrm{kpc}<1.2,\left\langle\mu_{0}>\right.$

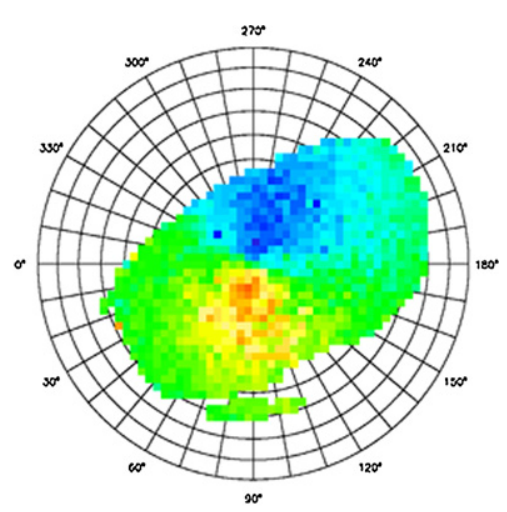

metol-rich, $1.8<0 /$ kpc $<2.2,<\mu_{0}>$

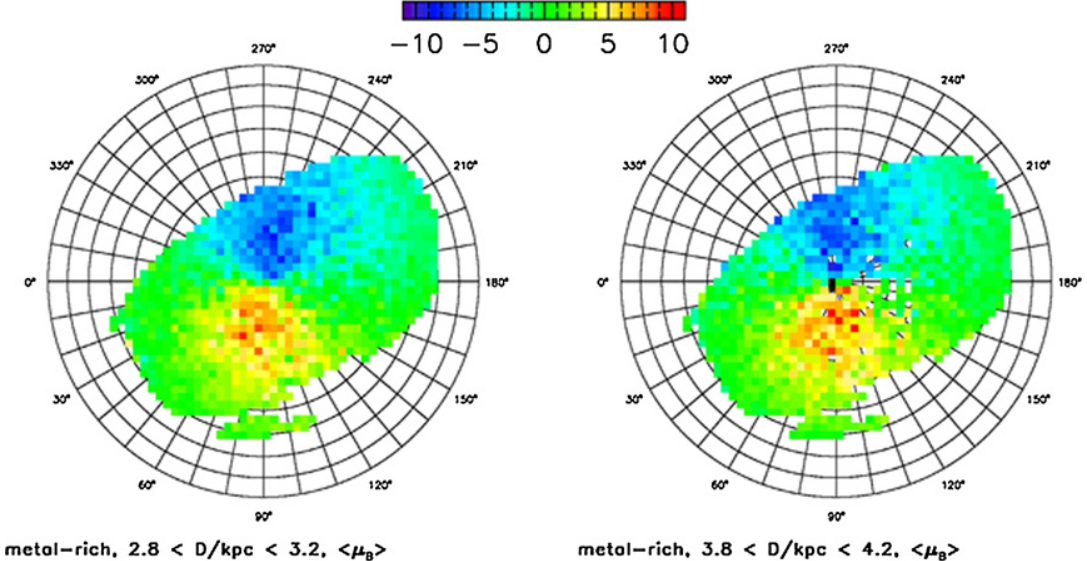

Figure 18. Analogous to Figure 17, except that the latitudinal proper motion is shown.

(A color version of this figure is available in the online journal.)

$(-20,180) \mathrm{km} \mathrm{s}^{-1}$, and $(20,220) \mathrm{km} \mathrm{s}^{-1}$ (for both we fixed $v_{\text {LSR }}-v_{\phi}^{\text {halo }}=200 \mathrm{~km} \mathrm{~s}^{-1}$, to make the models agree with the data toward the North Galactic Pole; see Figure 5). In order to distinguish these two models observationally, systematic errors in the radial velocities must be below $10 \mathrm{~km} \mathrm{~s}^{-1}$, and systematic errors in the observed proper motions must be below 1 mas $^{-1}{ }^{-1}$. These requirements are comparable to the systematic errors in our data set, so we can only state that the data are consistent with $v_{\mathrm{LSR}} \sim 200 \mathrm{~km} \mathrm{~s}^{-1}$ (or similarly, no halo rotation) to an accuracy of $\sim 20 \mathrm{~km} \mathrm{~s}^{-1}$. The proper-motion measurements from the Gaia survey (Perryman et al. 2001) will be sufficiently accurate to exploit the full potential of this method.

\subsection{The Kinematic-Parallax Relation}

Any constraint on our best-fit model parameters that uses proper motions is sensitive to the systematic errors in the distance scale obtained from our photometric-parallax relationship. However, since our model is a good fit to the radial-velocity data alone (see Figure 16), it is possible to estimate distance errors by minimizing the differences between the observed and modeled proper-motion distributions. Such a kinematic-parallax relation, derived from a combination of radial velocity and proper-motion data sets, was proposed for the solar neighborhood by Binney \& Tremaine (1987). Unlike in the solar neighborhood, our volume is sufficiently large that kinematics vary with position. Nevertheless, it is conceptually the same method; a dipole (in our case a more complex angular function) is fitted to the radial velocity and proper-motion distributions, and the ratio of the best-fit dipole magnitudes constrains the distance scale.
Using only low-metallicity halo stars, we obtain a distancescale error of $\sim 5 \%$ - our adopted absolute magnitudes should be $\sim 0.1$ mag brighter. This offset is consistent with the expected systematic errors in the calibration of the photometric-parallax relation (see I08). In other words, the adopted distance scale properly connects the radial and proper-motion distributions. This method provides a much weaker distance-scale constraints for disk stars than for halo stars, because the vertical velocity gradient in the disk leads to a degeneracy between errors in the distance scale and errors in the adopted disk velocity. The radial-velocity measurements do not constrain the velocity scale because the reference point depends on distance.

\section{SUMMARY AND DISCUSSION}

This is the first analysis based on SDSS data that simultaneously studies the kinematics of the halo and disk populations. Past studies of halo stars alone were performed by Sirko et al. (2004), Allende Prieto et al. (2006), Carollo et al. (2007), and Smith et al. (2009), while disk samples ranging from nearby $M$ stars to distant F/G stars have been studied by Bochanski et al. (2007b), West et al. (2008), and Fuchs et al. (2009). Throughout this paper, we have quantified the probability distribution function $p_{3}\left(\mu_{l}, \mu_{b}, v_{\text {rad }} \mid u-g, g, g-r, l, b\right)$, introduced in I08, that describes proper-motion and radial-velocity measurements in the $g$ versus $g-r$ color-magnitude diagram as a function of position on the sky and $u-g$ color. We have developed a simple empirical model with disk and halo components that map well to populations detected in the stellar density distribution (J08) and the metallicity distribution (I08). At distances accessible 


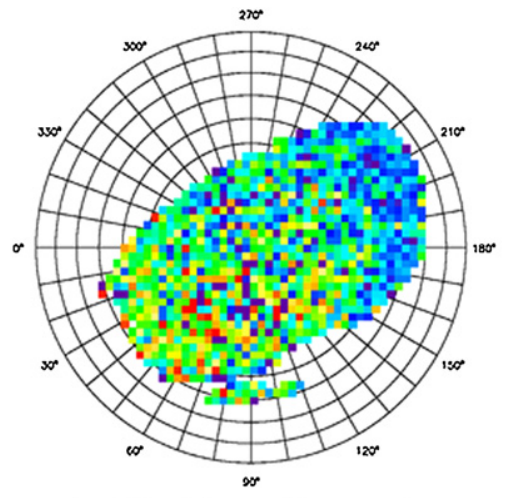

metal-rich, $0.8<0 / k p c<1.2, \mu_{2}:<$ residuols $>$

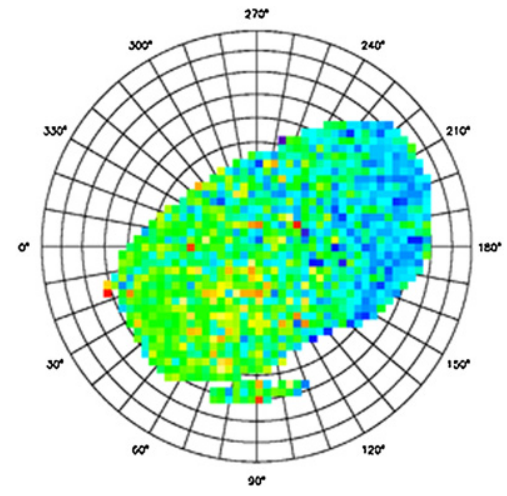

metol-rich, $1.8<\mathrm{D} / \mathrm{kpc}<2.2, \mu_{\mathrm{L}}$ : $<$ residuols $>$

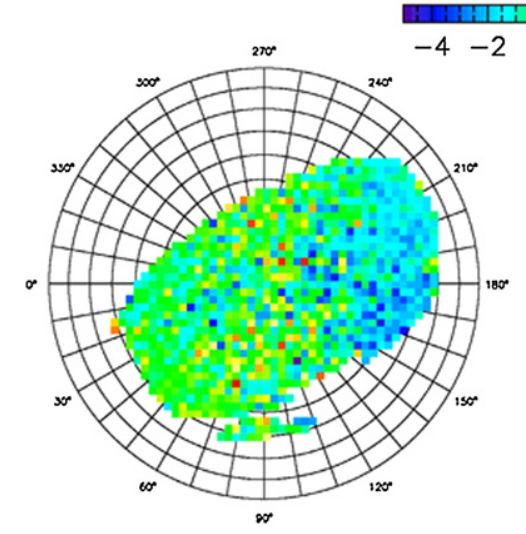

metal-rich, $2.8<\mathrm{D} / \mathrm{kpc}<3.2, \mu_{\mathrm{L}}$ : <residuols $>$

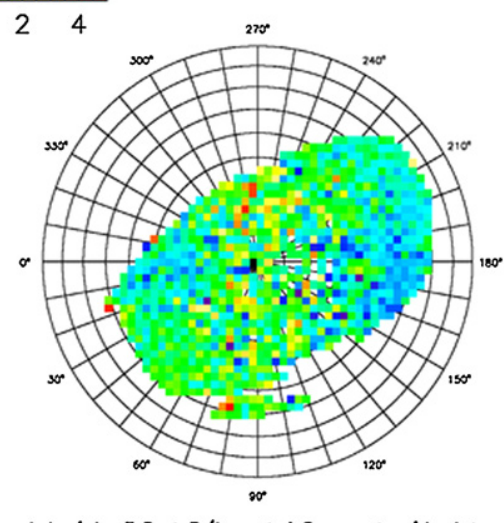

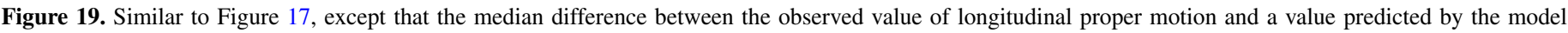

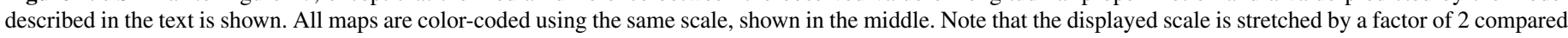
to the scale from Figure 17, in order to emphasize discrepancies.

(A color version of this figure is available in the online journal.)

to the HIPPARCOS survey ( $<100 \mathrm{pc}$ ), we obtain encouraging agreement with results from Dehnen \& Binney (1998), Dehnen (1998), and Nordström et al. (2004). The extension of kinematic mapping to distances up to $\sim 10 \mathrm{kpc}$ with millions of stars represents a significant observational advance, and delivers powerful new constraints on the dynamical structure of the Galaxy. In less than two decades, the observational material for such in situ mapping has progressed from first pioneering studies based on only a few hundred objects (Majewski 1992), to over a thousand objects (Chiba \& Beers 2000), to the massive data set discussed here.

\subsection{Disk Kinematics}

The disk kinematics of the Milky Way are dominated by rotation with a smooth vertical gradient. Our analysis extends the early measurements of this gradient (e.g., Murray 1986; Majewski 1992; Chiba \& Beers 2000) to vastly larger sky area and to a much larger distance range. The mean rotational velocity and the three velocity dispersions for disk stars can be modeled as simple functions of the form $a+b|Z|^{c}$ (see Equations (19) and (20), and Table 1). The rotational-velocity distribution for the disk component is non-Gaussian, and can be formally modeled as a sum of two Gaussian components with fixed normalization ratio for $0.1 \lesssim|Z| / \mathrm{kpc} \lesssim 4$. The fact that the normalization ratio of these two components does not vary with $Z$ is at odds with the standard disk decomposition into thin- and thick-disk components (see also Sections 3.4.4 and 4.2.1 in I08). Based on N-body simulations performed by Roškar et al. (2008), Loebman et al. (2008) argued that the absence of a velocity-metallicity correlation at the thin/thickdisk boundary, pointed out by I08, may be due to a combination of a strong vertical age gradient and the radial migration of stars (see also Schönrich \& Binney 2009). A more detailed study will be presented in S. Loebman et al. (2010, in preparation). A significant vertical age gradient for disk stars is also supported by an analysis of active $\mathrm{M}$ dwarfs presented in Bochanski et al. (2007b). Such an age gradient, together with the measured velocity dispersion-age correlations for local disk stars (e.g., Nordström et al. 2004; Rocha-Pinto et al. 2004; West et al. 2008), may be responsible for the measured increase of velocity dispersions with distance from the Galactic plane.

Close to the plane, the proper-motion data imply a complex multi-modal velocity distribution that is inconsistent with a description based on a simple Schwarzschild ellipsoid. It is reassuring that we obtained a velocity-distribution morphology very similar to that obtained by Dehnen (1998) using statistical deprojection of the HIPPARCOS data, and directly by Nordström et al. (2004) using a subsample of HIPPARCOS stars with full three-dimensional velocity information. In addition, our results for the first and second moments of the velocity distribution for nearby $M$ stars agree with analogous results obtained recently by Fuchs et al. (2009). The orientation of the velocity ellipsoid is strongly affected by multi-modal structure, so one should take care in its interpretation. We discuss these issues in more detail in A. Kowalski et al. (2010, in preparation). 

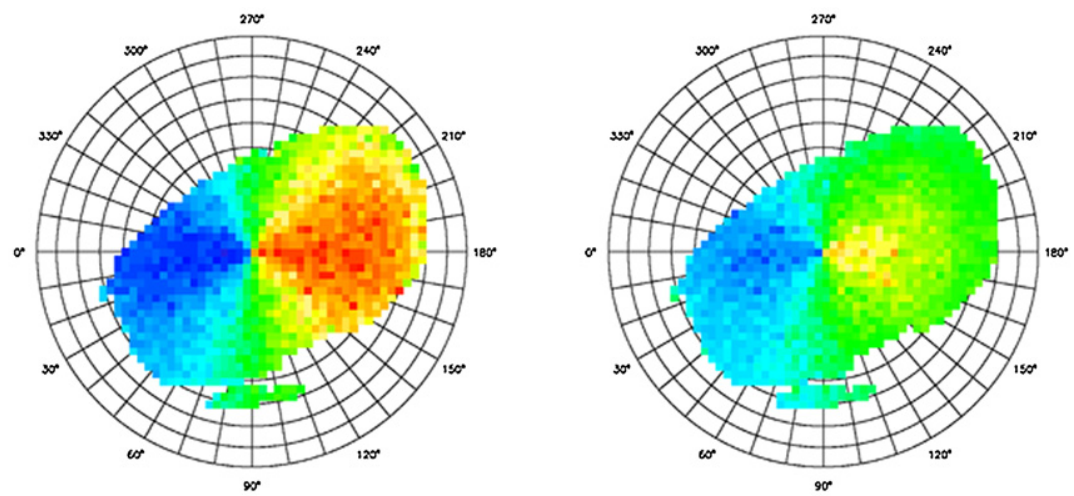

metal-poor, $3.5<\mathrm{D} / \mathrm{kpc}<4.5,\left\langle\mu_{\mathrm{c}}\right\rangle \quad$ metol-rich, $\left.3.5<\mathrm{D} / \mathrm{kpc}<4.5 .<\mu_{\mathrm{L}}\right\rangle$

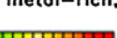

$-10-5 \quad 0 \quad 5 \quad 10$
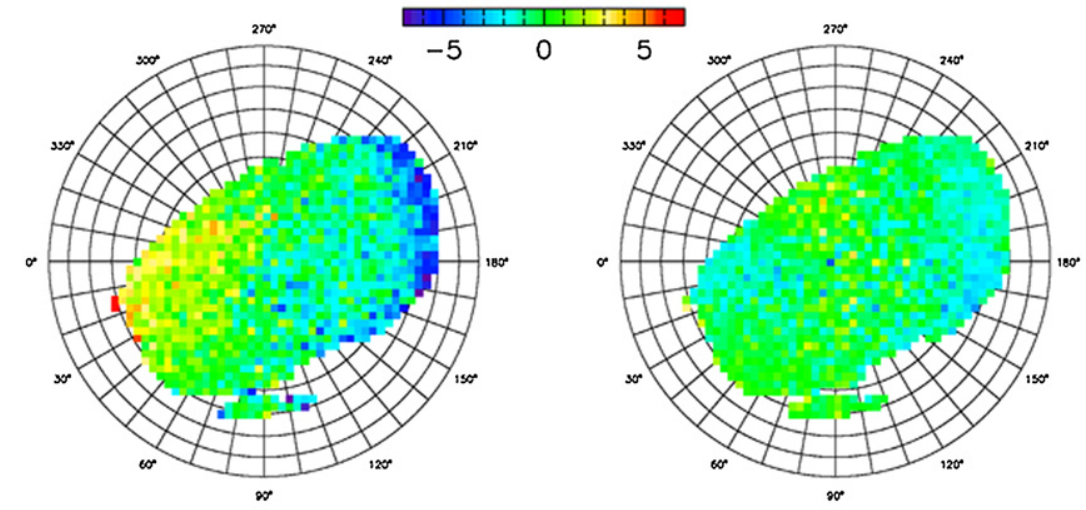

metol-poor, $3.5<\mathrm{D} / \mathrm{kpc}<4.5, \mu_{i}$ : <residuols $>$

metol-rich, $3.5<\mathrm{D} / \mathrm{kpc}<4.5, \mu_{\mathrm{n}}$ : <residuols $>$

Figure 20. Similar to Figures 17 and 19, except that the behavior of high-metallicity (left) and low-metallicity (right) stars is compared in a single distance bin $(3.5-4.5 \mathrm{kpc})$. The top two panels show the median longitudinal proper motion, and the two bottom panels show the median difference between the observed and model-predicted values. An analogous figure for the latitudinal proper motion has similar characteristics.

(A color version of this figure is available in the online journal.)

\subsection{Halo Kinematics}

Our results for the velocity distribution of halo stars are in excellent agreement with Smith et al. (2009). They used propermotion measurements based only on SDSS data, and thus have significantly different, and most likely much smaller, systematic errors than the SDSS-POSS proper-motion measurements analyzed here. The much larger size of the Munn et al. (2004) catalog analyzed here allows us to rule out the possibility that the Smith et al. result was biased by local substructure. The close agreement of our results for the orientation and size of the halo velocity ellipsoid (we obtained $\sigma_{r}^{H}=141 \mathrm{~km} \mathrm{~s}^{-1}$, $\sigma_{\theta}^{H}=75 \mathrm{~km} \mathrm{~s}^{-1}$, and $\sigma_{\phi}^{H}=85 \mathrm{~km} \mathrm{~s}^{-1}$, while their values are $142 \mathrm{~km} \mathrm{~s}^{-1}, 77 \mathrm{~km} \mathrm{~s}^{-1}$, and $81 \mathrm{~km} \mathrm{~s}^{-1}$, respectively) are encouraging (see also Carollo et al. 2009). Their estimated errors of $2 \mathrm{~km} \mathrm{~s}^{-1}$ apparently do not include systematic effects (such as errors in photometric parallax; both studies used the same calibration from I08) - based on our Monte Carlo simulations, we believe that the true errors cannot be smaller than $\sim 5 \mathrm{~km} \mathrm{~s}^{-1}$. Additional independent evidence for the tilt of the velocity ellipsoid comes from the RAVE survey (Siebert et al. 2008). These measurements of the velocity ellipsoid for halo stars represent a strong constraint for the shape of gravitational potential, as discussed by, e.g., Amendt \& Cuddeford (1991), Kent \& de Zeeuw (1991), and Smith et al. (2009).

We note that Majewski (1992) measured a retrograde halo rotation using stars observed toward the North Galactic Pole (in our nomenclature, he obtained a mean rotational velocity $v_{\Phi}=$ $50 \pm 16 \mathrm{~km} \mathrm{~s}^{-1}$ ). A star-by-star comparison of his data and the data analyzed here showed that photometric and proper-motion measurements agree within the stated errors. The main reason for different conclusions about halo rotation are the different distance scales: his distances are on average 30\% larger than our distances, resulting in larger tangential velocities. The Carollo et al. (2007) claim for a large outer-halo retrograde rotation, further refined by Carollo et al. (2009) (in our nomenclature, a mean rotational velocity $v_{\Phi}=80 \pm 13 \mathrm{~km} \mathrm{~s}^{-1}$ ), remains intact, as their distance scale is similar to ours.

The kinematic measurements for halo stars presented here should not be extrapolated beyond the sample distance limit of $10 \mathrm{kpc}$. For example, using 241 halo objects, including stars, globular clusters, and satellite galaxies, Battaglia et al. (2005) detected a continuous decline of the radial-velocity dispersion beyond a Galactocentric radius of $\sim 30 \mathrm{kpc}$, from about $120 \mathrm{~km} \mathrm{~s}^{-1}$ to $50 \mathrm{~km} \mathrm{~s}^{-1}$ at $\sim 120 \mathrm{kpc}$. In addition, the distance limit of our sample, together with the decreasing sensitivity of the photometric-metallicity indicator for $[\mathrm{Fe} / \mathrm{H}]<$ -2.5 , prevents us from robustly testing the possible halo dichotomy discussed by Carollo et al. $(2007,2009)$.

\subsection{Kinematic Substructure}

The model developed here can be used to search for kinematic substructure with a low contrast level. For example, Schlaufman et al. (2009) had to generate a background model when searching 

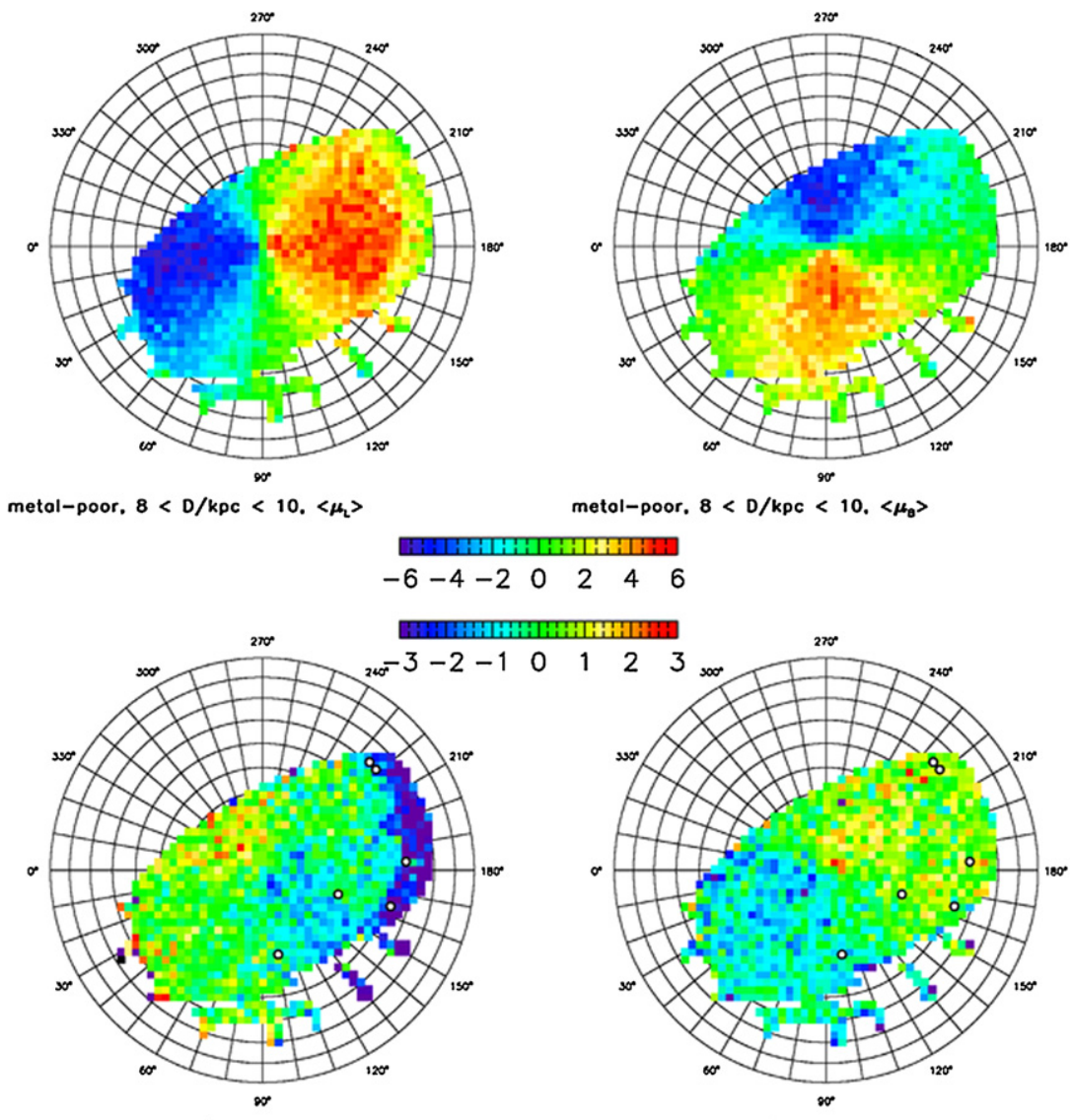

metol-poor, $8<\mathrm{D} / \mathrm{kpc}<10,\left\langle\mu_{\theta}\right\rangle$

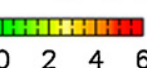

metol-poor, $8<D / k p c<10, \mu_{\mathrm{L}}$ : <residuols $>$

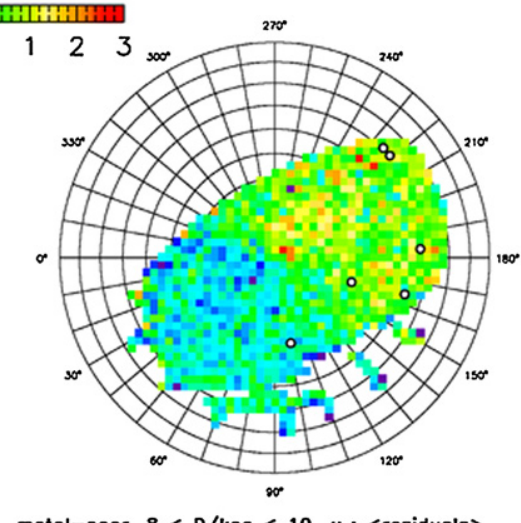

Figure 21. Similar to Figure 20, except that the behavior of low-metallicity stars from the 8-10 kpc distance bin is analyzed. The top two panels show the median longitudinal (left) and latitudinal (right) proper motions, and the two bottom panels show the median difference between the observed and model-predicted values. The

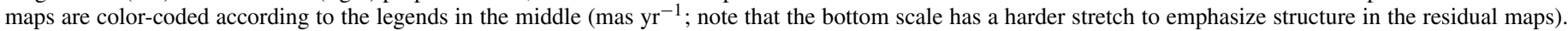
The two bottom panels display very similar morphology to systematic proper-motion errors shown in the two left panels in Figure 1. In the bottom panels, the white symbols show the positions of the six northern cold substructures identified by Schlaufman et al. (2009).

(A color version of this figure is available in the online journal.)
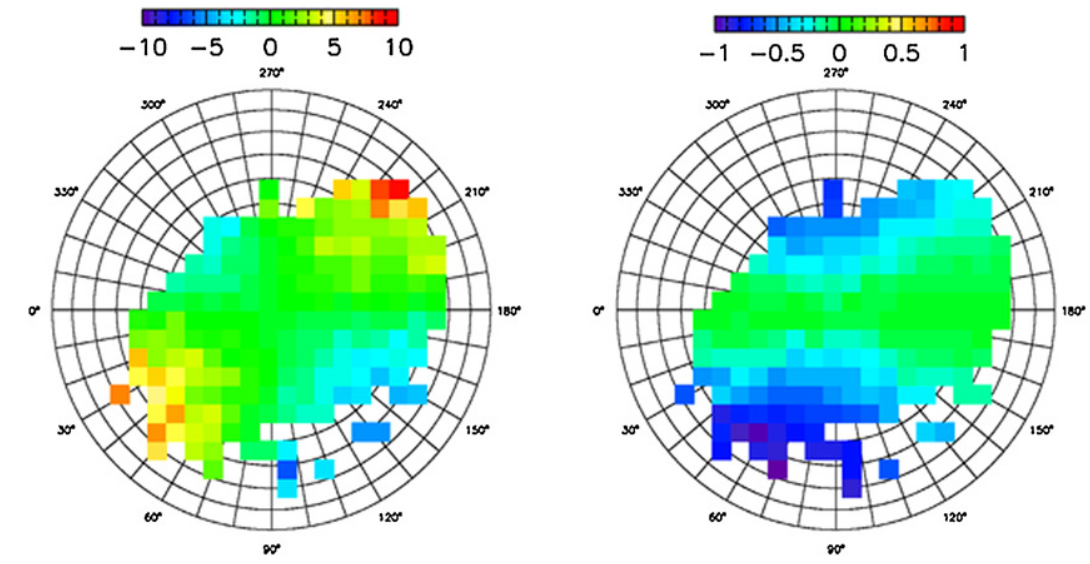

metol-poor, $2<\mathrm{D} / \mathrm{kpc}<7$, resid. $(M 1-M 2)$ for $<\mathrm{v}_{\text {rod }}>$

metol-poor, $2<\mathrm{D} / \mathrm{kpc}<7$, doto $<\mu_{1}^{\mu} 1-\mu_{1}^{\mathrm{N}} 2>$

Figure 22. Comparison of radial-velocity residuals (left panel; analogous to the top-right panel in Figure 16) and longitudinal proper-motion residuals (right panel; analogous to the bottom-left panel in Figure 20, except for the larger distance range) for two halo models with $\left(v_{\phi}^{\text {halo }}, v_{\mathrm{LSR}}\right)=(-20,180) \mathrm{km} \mathrm{s}^{-1}$, and $(20,220) \mathrm{km} \mathrm{s}^{-1}$. Note that we set $v_{\mathrm{LSR}}-v_{\phi}^{\text {halo }}=200 \mathrm{~km} \mathrm{~s}^{-1}$. The residuals are color-coded according to the legend above each panel (units are km s${ }^{-1}$ for the left panel and mas yr ${ }^{-1}$ for the right panel). In order to distinguish these models, systematic errors in radial velocity must be below $10 \mathrm{~km} \mathrm{~s}^{-1}$, and systematic errors in proper motion must be below 1 mas $\mathrm{yr}^{-1}$.

(A color version of this figure is available in the online journal.)

for cold streams using radial-velocity data; a similar study for the solar neighborhood was performed by Klement et al. (2009). A global model-based description is especially important when using large numbers of proper-motion measurements to search for substructure. While radial-velocity data are superior at large distances, searches based on proper motions should be better 
within a few kpc, due to the high completeness and much larger sample size. A user-friendly interface to our model code Galfast, ${ }^{32}$ which allows generation of mock catalogs in an arbitrary direction (or across the entire sky) and to an arbitrary depth, will be described elsewhere (M. Jurić et al. 2010, in preparation).

The Monoceros stream is clearly detected as a major outlier from the smooth model presented here. We have not found any other large kinematic substructure within $10 \mathrm{kpc}$ that deviates at a detectable level. Other deviations from the smooth model predictions are likely due to systematic errors in the propermotion and radial-velocity measurements. However, since the main goal of this paper was to quantify the overall kinematic behavior, the emphasis of our analysis was on the first and second moments of the kinematic quantities. It is likely that more sophisticated statistical methods, such as those discussed by Schlaufman et al. (2009) and Klement et al. (2009), will be more efficient in searching for substructure. As an example, we have verified that a moving group discovered by Majewski (1992) is reproducible with our data.

\subsection{Future Surveys}

The results presented here will be greatly extended by several upcoming large-scale, deep optical surveys, including the Dark Energy Survey (Flaugher 2008), Pan-STARRS (Kaiser et al. 2002), and the Large Synoptic Survey Telescope (LSST; Ivezić et al. 2008b). These surveys will extend the faint limit of this sample and that of the upcoming Gaia mission (Perryman et al. 2001; Wilkinson et al. 2005) by 4-6 mag. For example, LSST will obtain proper-motion measurements of comparable accuracy to those of Gaia at their faint limit, and smoothly extend the error versus magnitude curve deeper by $5 \mathrm{mag}$ (for details see L. Eyer et al. 2010, in preparation). With its $u$-band data, LSST will enable studies of metallicity and kinematics using the same stars out to a distance of $\sim 100 \mathrm{kpc}(\sim 200$ million F/G mainsequence stars brighter than $g=23.5$, for a discussion see I08). By comparison, the best measurements of the outer-halo radialvelocity dispersion to date are based on from several hundred (Battaglia et al. 2005) to several thousand (Xue et al. 2008) objects. These upcoming studies are thus certain to provide valuable new information about the formation and evolution of our Galaxy.

We thank Vladimir Korchagin for making his code for calculating equilibrium velocity dispersion profile available to us. Ž. Ivezić and B. Sesar acknowledge support by NSF grants AST615991 and AST-0707901, and by NSF grant AST-0551161 to LSST for design and development activity. J. Dalcanton acknowledges NSF CAREER grant AST-02-38683. D. Schneider acknowledges support by NSF grant AST-06-07634. Allende Prieto acknowledges support by NASA grants NAG5-13057 and NAG5-13147. T.C. Beers, Y.S. Lee, and T. Sivarani acknowledge partial support from PHY 08-22648: Physics Frontier Center/Joint Institute for Nuclear Astrophysics (JINA), awarded by the U.S. National Science Foundation. P. Re Fiorentin acknowledges support through the Marie Curie Research Training Network ELSA (European Leadership in Space Astrometry) under contract MRTN-CT-2006-033481. M.J. acknowledges support by NASA grant NNXO-8AH83G and NSF grant AST-0807444. We acknowledge the hospitality of the KITP at the University of California, Santa Barbara, where part

\footnotetext{
32 Please see http://mwscience.net/trac/wiki/galfast
}

of this work was completed (supported by NSF grant PHY0551164). Fermilab is operated by Fermi Research Alliance, LLC under Contract No. DE-AC02-07CH11359 with the United States Department of Energy. Funding for the SDSS and SDSSII has been provided by the Alfred P. Sloan Foundation, the Participating Institutions, the National Science Foundation, the U.S. Department of Energy, the National Aeronautics and Space Administration, the Japanese Monbukagakusho, the Max Planck Society, and the Higher Education Funding Council for England. The SDSS Web Site is http://www.sdss.org/. The SDSS is managed by the Astrophysical Research Consortium for the Participating Institutions. The Participating Institutions are the American Museum of Natural History, Astrophysical Institute Potsdam, University of Basel, University of Cambridge, Case Western Reserve University, University of Chicago, Drexel University, Fermilab, the Institute for Advanced Study, the Japan Participation Group, Johns Hopkins University, the Joint Institute for Nuclear Astrophysics, the Kavli Institute for Particle Astrophysics and Cosmology, the Korean Scientist Group, the Chinese Academy of Sciences (LAMOST), Los Alamos National Laboratory, the Max-Planck-Institute for Astronomy (MPIA), the Max-Planck-Institute for Astrophysics (MPA), New Mexico State University, Ohio State University, University of Pittsburgh, University of Portsmouth, Princeton University, the United States Naval Observatory, and the University of Washington.

\section{APPENDIX A}

\section{THE REVISED SDSS METALLICITY SCALE}

Analysis of the metallicity and kinematics of halo and disk stars by $\mathrm{I} 08$ utilized photometric-metallicity estimates for $\mathrm{F} / \mathrm{G}$ stars with $0.2<g-r<0.6$. Their mapping function from the $g-r$ versus $u-g$ color-color diagram to metallicity was calibrated using stars with spectroscopic metallicities distributed in SDSS Data Release 6. At that time, high-metallicity stars required for the calibration of methods implemented in the automated spectroscopic pipeline (SEGUE Stellar Parameters Pipeline; Beers et al. 2006) were not available. Between Data Releases 6 and 7, the required data were collected and the new calibration resulted in the revised spectroscopic-metallicity values distributed with Data Release 7 (Lee et al. 2008a, 2008b; Allende Prieto et al. 2008).

Here, we recalibrate the photometric-metallicity estimator using updated spectroscopic metallicities from Data Release 7. In addition, we re-derive the parts of the I08 analysis that are most affected by this change in the metallicity scale.

\section{A.1. The Updated Photometric-Metallicity Estimator}

As shown in Figure A.1, the largest difference between the SDSS spectroscopic-metallicity values distributed with Data Releases 6 and 7 is, as expected, at the high-metallicity end. In particular, the abrupt cutoff in the metallicity distribution at $[\mathrm{Fe} / \mathrm{H}] \sim-0.5$ (see Figure 9 in I08) is no longer present, and the distribution extends to values as high as $[\mathrm{Fe} / \mathrm{H}] \sim-0.2$ (the distances for the stars shown range from $\sim 1 \mathrm{kpc}$ to $\sim 7 \mathrm{kpc}$ ).

We proceed to re-derive the photometric-metallicity calibration using the same selection criteria and the same methodology as in I08. The new data set admits a slightly simpler function-the double definition of the $x$-axis is no longer required, 

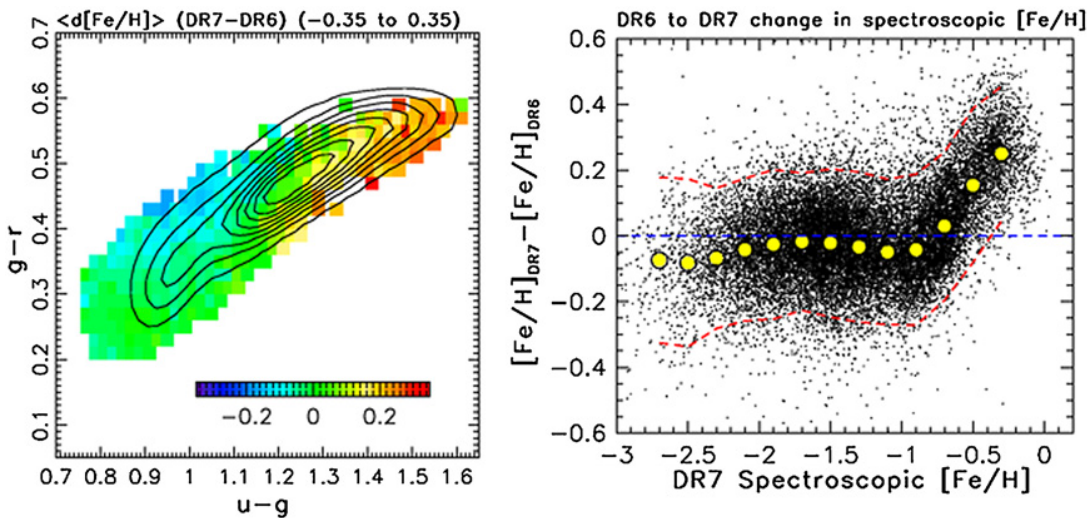

Figure A.1. Summary of the differences between SDSS spectroscopic-metallicity values distributed with Data Releases 6 and 7. The left panel shows the median difference between the DR7 and DR6 values for $0.02 \times 0.02 \mathrm{mag}^{2}$ bins in the $g-r$ vs. $u-g$ color-color diagram, color-coded according to the legend shown in the panel. The largest differences of $0.2-0.3$ dex are seen in the top-right corner, which corresponds to high metallicities. The right panel shows the difference in metallicities as a function of the new DR7 values. Individual stars are shown as small dots, and the median values of the difference are shown as large circles. The two dashed lines mark the $\pm 2 \sigma$ envelope around the medians, where $\sigma$ is the rms scatter ( $\sim 0.1$ dex, due to software updates) estimated from the interquartile range. The median differences are larger than 0.1 dex only at the high-metallicity end $([\mathrm{Fe} / \mathrm{H}]>-0.6)$.

(A color version of this figure is available in the online journal.)

and the new expression is

$$
\begin{aligned}
{[\mathrm{Fe} / \mathrm{H}]_{\mathrm{ph}}=} & A+B x+C y+D x y+E x^{2}+F y^{2}+G x^{2} y \\
& +H x y^{2}+I x^{3}+J y^{3}
\end{aligned}
$$

with $x=(u-g)$ and $y=(g-r)$. The best-fit coefficients are $(A-J)=(-13.13,14.09,28.04,-5.51,-5.90,-58.68$, $9.14,-20.61,0.0,58.20)$. Note that the coefficient for $x^{3}, I$, is to zero. We removed this term because it was producing too much curvature at the right end (red $u-g$ ) of the best-fit map.

We estimate that an upper limit on the intrinsic metallicity scatter for fixed noiseless $u-g$ and $g-r$ colors (presumably due to limited sensitivity of broadband colors to metallicity variations) is about 0.1 dex. This value is estimated from the scatter in the difference between spectroscopic and photometric metallicities, discussed below. Unlike I08, who simply adopted the median metallicity value given by the above expression for each star, we draw photometric-metallicity estimates from a Gaussian distribution centered on the best-fit median value, and with a width of 0.1 dex. The main benefit to this is that we avoid hard edges in the photometric-metallicity distribution for stars close to the edges of the calibration region in the $g-r$ versus $u-g$ diagram.

The performance of the new map is qualitatively similar to that of the old map. The mean and rms values for the difference between spectroscopic and photometric metallicities as functions of the $g-r$ and $u-g$ colors are shown in the top two panels of Figure A.2. Typical systematic errors in the map (i.e., the median difference per pixel) are $\sim 0.1$ dex or smaller, and the scatter varies from $\sim 0.2$ dex at the high-metallicity end to $\sim 0.3$ dex at the low-metallicity end (note that this scatter includes a contribution from errors in both spectroscopic and photometric metallicities).

The above photometric-metallicity estimator is applicable for stars with $0.2<g-r<0.6$ and $-0.25+0.5(u-g)<g-r<$ $0.05+0.5(u-g)$; that is, for main-sequence $\mathrm{F}$ and $\mathrm{G}$ stars in the calibration region of the $g-r$ versus $u-g$ color-color diagram (top two panels of Figure A.2). For stars with spectroscopic metallicity $[\mathrm{Fe} / \mathrm{H}]>-2.2$, the distribution of the difference between spectroscopic and photometric metallicities is welldescribed by a Gaussian with a width of 0.26 dex (see the bottom-right panel in Figure A.2).
It should be noted that the performance of the photometricmetallicity estimator deteriorates at the low-metallicity end because the $u-g$ color becomes insensitive to decreases in metallicity. As shown in the bottom-left panel of Figure A.2, the photometric metallicity saturates at $[\mathrm{Fe} / \mathrm{H}] \sim-2$ for smaller values of spectroscopic metallicity. Even at $[\mathrm{Fe} / \mathrm{H}]=-2$, the true metallicity is overestimated by $0.2-0.3$ dex, and by $[\mathrm{Fe} / \mathrm{H}]=-3$ this bias is as large as $1 \mathrm{dex}$ (the photometricmetallicity values never become significantly lower than $[\mathrm{Fe} / \mathrm{H}]=-2$ ). This shortcoming could be partially alleviated by employing more accurate $u$-band photometry (say, with errors of $0.01 \mathrm{mag}$ instead of $0.03 \mathrm{mag}$, as used here), but probably not for metallicities lower than $[\mathrm{Fe} / \mathrm{H}]=-2.5$. Fortunately, the low-metallicity inner-halo stars within SDSS reach have a median metallicity of $[\mathrm{Fe} / \mathrm{H}] \sim-1.5$ (I08), so for the majority of stars the photometric metallicities are robust. Another important note is that, despite the improvement at the high-metallicity end, the calibration range only extends to $[\mathrm{Fe} / \mathrm{H}] \sim-0.2$. Our calibration sample did not include young, metal-rich main-sequence stars $(u-g>1.1$ and $g-r<0.3)$. For this reason, our polynomial model underestimates true metallicities by $0.2-0.3$ dex at $[\mathrm{Fe} / \mathrm{H}]=0$, and probably more for $[\mathrm{Fe} / \mathrm{H}]>0$. Any result relying on higher metallicities should be interpreted with caution (especially at low Galactic latitudes where the uncertain interstellar medium extinction may strongly affect the estimated metallicities). We plan to extend our calibration further into the metal-rich domain by employing data from the SDSS-III SEGUE-2 survey (Yanny et al. 2009), and further refinements of the SEGUE Stellar Parameters Pipeline, which are now underway.

\section{A.2. Tomography II Reloaded}

I08 pointed out several aspects of their analysis that may have been affected by the metallicity "compression" at the high-metallicity end in DR6. We repeated their full analysis and report here on those aspects where differences warrant discussion.

The "hard" upper limit on photometric-metallicity estimates at the high-metallicity end $([\mathrm{Fe} / \mathrm{H}] \sim-0.5)$ with the DR6 calibration is best seen in the bottom-left panel in Figure 9 from I08. We reproduce that map of the conditional metallicity distribution in the top-left panel of Figure A.3. As expected, the 

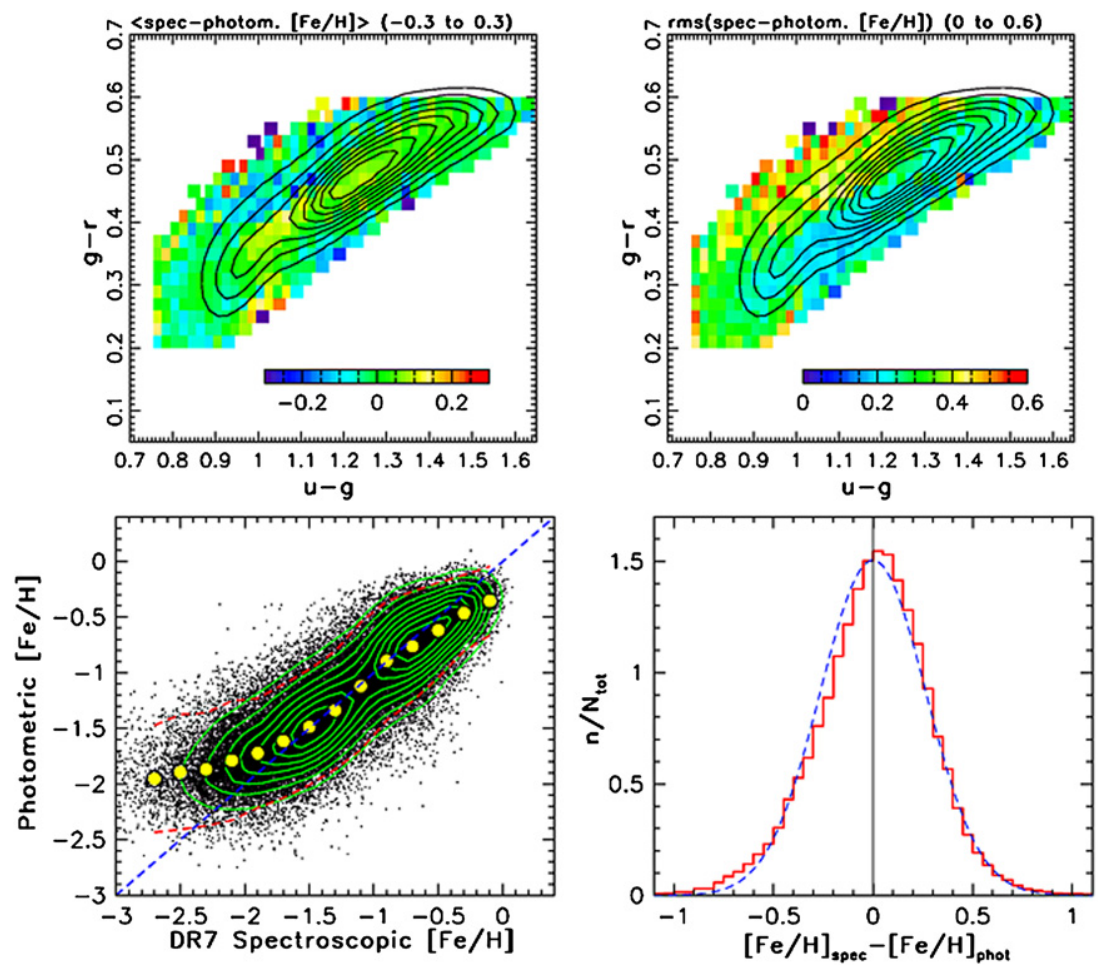

Figure A.2. Color-coded map in the top-left panel shows the median difference between spectroscopic and revised photometric metallicities for $\sim 50,000$ stars from SDSS Data Release 7. The median value is zero, and the rms scatter is 0.07 dex. The contours show the distribution of stars with $r<20$ and at high Galactic latitudes. The top-right panel shows the rms scatter of the difference between spectroscopic and photometric metallicities in each pixel. The top two panels are analogous to the bottom two panels in Figure 2 from I08. The bottom-left panel shows the photometric metallicity as a function of the spectroscopic metallicity. Individual stars are shown by small dots, and the median values of the difference are shown by large circles. The distribution of stars is shown as linearly spaced contours. Note that the photometric metallicity saturates at $[\mathrm{Fe} / \mathrm{H}] \sim-2$ at the low-metallicity end. The histogram in the bottom-right panel shows the distribution of the difference between spectroscopic and photometric metallicities for stars with spectroscopic metallicity $[\mathrm{Fe} / \mathrm{H}]>-2.2$. A best-fit Gaussian centered on zero and with a width of 0.26 dex is shown by the dashed line.

(A color version of this figure is available in the online journal.)

metallicity distribution of disk stars within $2 \mathrm{kpc}$ of the Galactic plane now extends to $[\mathrm{Fe} / \mathrm{H}] \sim 0$.

In the new calibration, the parameters describing the variation of the median metallicity for disk stars as a function of the distance from the Galactic plane,

$$
\mu_{D}(Z)=\mu_{\infty}+\Delta_{\mu} \exp \left(-|Z| / H_{\mu}\right) \operatorname{dex},
$$

are also changed. The updated values are $H_{\mu}=0.5 \mathrm{kpc}, \mu_{\infty}=$ -0.82 , and $\Delta_{\mu}=0.55$ (the old values were $H_{\mu}=1.0 \mathrm{kpc}$, $\mu_{\infty}=-0.78$, and $\Delta_{\mu}=0.35$ ). The best-fit values of $\mu_{\infty}$ and $\Delta_{\mu}$ are accurate to $\sim 0.05$ dex. Values of $H_{\mu}$ in the range 350-700 pc are consistent with the data-the decrease in $H_{\mu}$ is required by the local constraint, $\mu_{D}(Z)=-0.2$ (Nordström et al. 2004; Allende Prieto et al. 2004).

An interesting result from I08 was the detection of disk stars at a distance from the Galactic plane as large as $\sim 6 \mathrm{kpc}$ (see their Figure 10). A peak at $[\mathrm{Fe} / \mathrm{H}]=-0.5$ in the metallicity distribution of stars at those distances was another manifestation of the metallicity "compression." As demonstrated in the topright panel of Figure A.3, this peak is not present when using the revised calibration. However, there is still statistical evidence that disk stars exist at these large distances from the plane: about $5 \%$ of stars in the $5<Z<7 \mathrm{kpc}$ bin are consistent with disk stars, in agreement with extrapolation of the exponential profile derived from stellar counts. Lee \& Beers (2009) and Carollo et al. (2009) have also commented on signatures of thin-disk-like chemistry and kinematics for a small fraction of stars several kpc above the Galactic plane.

Perhaps the most intriguing result of I08 was the nondetection of a correlation between rotational velocity and metallicity for disk stars at $Z \sim 1 \mathrm{kpc}$. At such distances from the Galactic plane, the counts of thin- and thick-disk stars are expected to be similar. Since traditionally the thickdisk component is associated with a larger velocity lag and lower metallicities, a fairly strong and detectable correlation was expected (see I08 for details). The two bottom panels in Figure A. 3 demonstrate that such a correlation is still undetected, although the photometric-metallicity range now extends to higher values (up to $[\mathrm{Fe} / \mathrm{H}] \sim-0.2$ ).

The higher metallicity values obtained with the recalibrated relation have a quantitative effect on the best-fit metallicity distributions shown in Figure 7 of I08. Using the same methodology, we reproduce the metallicity distributions with the new calibration in Figure A.4. I08 modeled the non-Gaussian disk metallicity distribution using a sum of two Gaussians with a fixed amplitude ratio (1.7:1), fixed difference of the mean values $(0.14 \mathrm{dex})$, and fixed widths $(0.21 \mathrm{dex}$ and $0.11 \mathrm{dex})$, which "slides" as a function of $Z$, according to Equation (A2). We find that the only significant change is an increase to the width of the second Gaussian to 0.21 dex, which accounts for the extension of the metallicity distribution to higher values. Only minor changes are required for the best-fit halo metallicity distribution (see Table 3 in I08): the median halo metallicity is now -1.46 

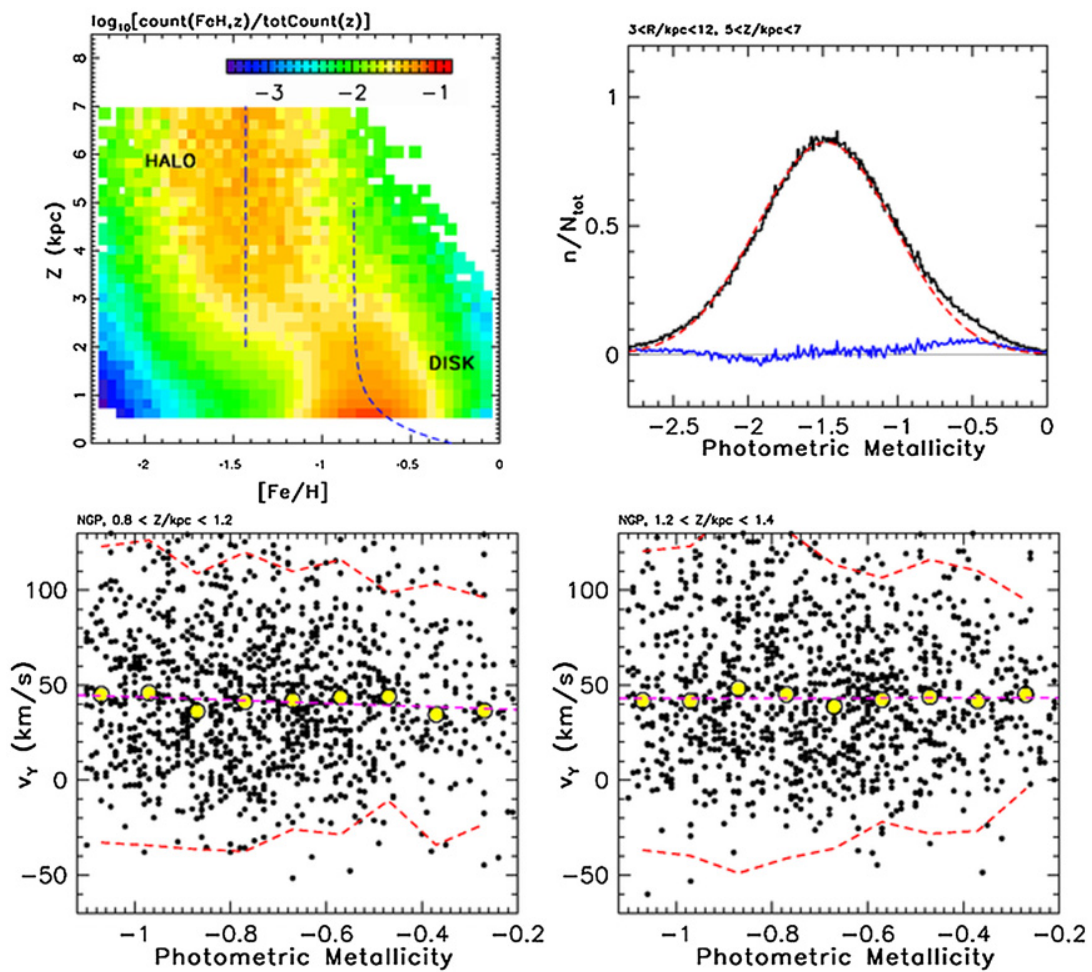

Figure A.3. Conditional metallicity distribution as a function of distance from the Galactic plane (top left), analogous to the bottom-left panel of Figure 9 from I08. Note that the new photometric metallicities include $[\mathrm{Fe} / \mathrm{H}]>-0.4$. The dashed line, which shows the median disk metallicity, is also revised (see the text). The top-right panel is analogous to Figure 10 from I08, and shows the metallicity distribution for stars with $5<Z<7 \mathrm{kpc}$, where $Z$ is the distance from the Galactic plane. Note that the photometric-metallicity artifact at $[\mathrm{Fe} / \mathrm{H}]=-0.5$ discussed by $\mathrm{I} 08$ is no longer present. However, there is still evidence that disk stars exist at such large distances from the plane. The bottom two panels show the heliocentric rotational velocity for disk stars in two thin $Z$ slices, and are analogous to the bottom-right panel in Figure 16 from I08. Note that the correlation between velocity and metallicity is still absent.

(A color version of this figure is available in the online journal.)
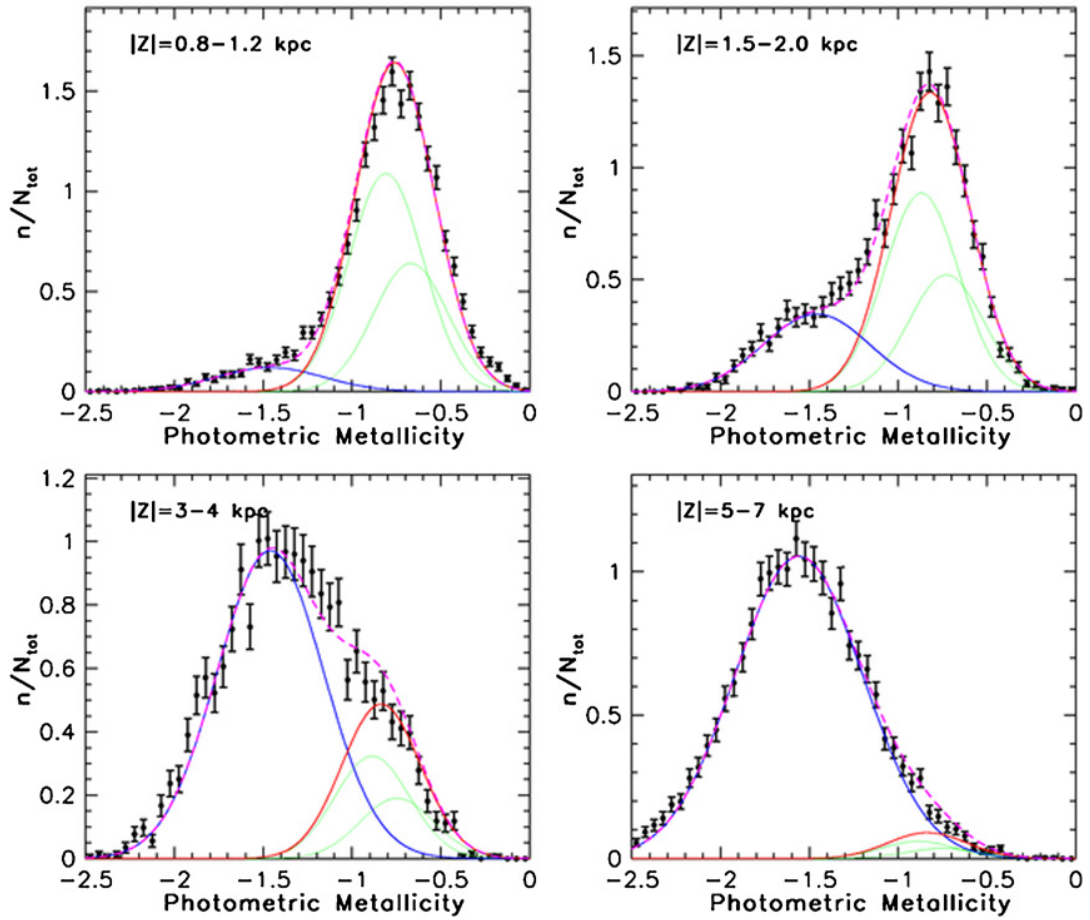

Figure A.4. Analogous to Figure 7 from I08. The symbols with error bars show the metallicity distribution for stars with $0.2<g-r<0.4,7<R<9$ kpc, and distances from the Galactic plane as marked, where $R$ is the Galactocentric cylindrical radius. The behavior is qualitatively similar to that seen in I08. The only significant quantitative difference is in the model for the metallicity distribution of disk stars (see the text).

(A color version of this figure is available in the online journal.) 
in the first three bins, and -1.56 in the most distant $Z$ bin, and its width changed from 0.32 dex to $0.36 \mathrm{dex}$ in the last bin. We note somewhat less scatter of the data points around the best-fit functions with the recalibrated data set. To summarize, the revised best-fit parameters that describe halo and disk metallicity distributions are as follows.

1. The halo metallicity distribution is spatially invariant and well-described by a Gaussian distribution centered on $[\mathrm{Fe} / \mathrm{H}]=-1.46$, with a width $\sigma_{H}=0.30 \mathrm{dex}$ (not including measurement errors). For $|Z| \lesssim 10 \mathrm{kpc}$, an upper limit on the halo radial-metallicity gradient is $0.005 \mathrm{dex} \mathrm{kpc}^{-1}$.

2 . The disk metallicity distribution varies with $Z$ such that its shape remains fixed, while its median, $\mu_{D}$, varies as given by Equation (A2) (with best-fit parameter values $H_{\mu}=0.5 \mathrm{kpc}, \mu_{\infty}=-0.82$, and $\left.\Delta_{\mu}=0.55\right)$. The shape of the disk metallicity distribution can be modeled as

$$
\begin{aligned}
p_{D}(x= & {[\mathrm{Fe} / \mathrm{H}] \mid Z)=0.63 G[x \mid \mu=a(Z), \sigma=0.2] } \\
& +0.37 G[x \mid \mu=a(Z)+0.14, \sigma=0.2], \quad(\mathrm{A})
\end{aligned}
$$

where the position $a$ and the median $\mu_{D}$ are related via $a(Z)=\mu_{D}(Z)-0.067$ (unless measurement errors are very large).

We point out that the asymmetry of the metallicity distribution for disk stars is now less pronounced (as implied by the same widths of the two best-fit Gaussian components). Nevertheless, due to our large sample size, the non-Gaussianity is detected at high significance. A remaining uncertainty is the error distribution of the photometric metallicities, which itself could account for such a deviation from Gaussianity. However, to the extent possible using a highly incomplete spectroscopic sample (cf. the bottom-right panel in Figure A.2 and discussion in I08), we are unable to quantitatively explain the observed deviation from Gaussianity as an artifact of the photometric-metallicity method.

\section{APPENDIX B}

\section{SQL QUERY EXAMPLE}

The following SQL query was used to select and download data for all SDSS stars with spectroscopic and propermotion measurements (see http://casjobs.sdss.org/CasJobs). SELECT

round(p.ra,6) as ra, round(p.dec,6) as dec, p.run, p.rerun, round(p.extinction_r,3) as rExt, round(p.psfMag_u,2) as upsf, -- comments are preceded by --

round(p.psfMag_g,2) as gpsf, -- rounding up round (p.psfMag_r,2) as rpsf, round (p.psfMag_i,2) as ipsf, round(p.psfMag_z,2) as zpsf, round(p.psfMagErr_u,2) as uErr, round (p.psfMagErr_g,2) as gErr, round (p.psfMagErr_r,2) as rErr, round(p.psfMagErr_i,2) as iErr, round (p.psfMagErr_z,2) as zErr, round (s.pmL,2) as pmL, round (s.pmB,2) as pmB, pmRaErr,

t.specObjID, t.plate, t.mjd, t.fiberid, t.feha, t.fehaerr,

t.logga, t.loggaerr, t.elodierv, t.elodierverr INTO mydb.pmSpec
FROM star $p$, propermotions $s$, sppParams $t$, specobjall q

WHERE

p.objID $=$ s.objID and s.match $=1$-- must have proper motion and t.specobjid = q.specobjid and q.bestobjid $=$ p.objid and s.sigra $<350$ and s.sigdec $<350--$ quality cut on pm and (p.flags \& ' $\left.4295229440^{\prime}\right)=0--$ see text for flag list and p.psfMag_r $>14.5$-- avoid saturation and p.psfMag_r $<20--$ practical faint limit for pm -- the end of query

The following SQL query was used to select and download data for all spectroscopically confirmed quasars with proper-motion measurements and redshifts in the range 0.5-2.5. SELECT

round(p.ra,6) as ra, round(p.dec,6) as dec, p.run, p.rerun, round(p.extinction_r,3) as rExt, round (p.psfMag_u,2) as upsf, round (p.psfMag_g,2) as gpsf, round (p.psfMag_r,2) as rpsf, round(p.psfMag_i,2) as ipsf, round (p.psfMagErr_u,2) as uErr, round (p.psfMagErr_g,2) as gErr, round (p.psfMagErr_r,2) as rErr, round (p.psfMagErr_i,2) as iErr, round (s.pmL,2) as pmL, round (s.pmB,2) as pmB, pmRaErr, q.specObjID, q.plate, q.mjd, q.fiberID, q.z, q.zErr, q.zConf, q.zWarning, q.specClass INTO mydb.pmQSO

FROM star p, propermotions $\mathrm{s}$, specobjall q WHERE

p.objID $=$ s.objID and s.match $=1$ and

q.bestobjid $=$ p.objid and s.sigra $<350$ and

s.sigdec $<350$-- per s.s and (p.flags \&

$\left.{ }^{\prime} 4295229440^{\prime}\right)=0$ and p.psfMag_r $>14.5$ and

p.psfMag_r $<20$ and q.z $>0.5--$ redshift

limits and $q . z<2.5$

-- the end of query

\section{REFERENCES}

Abazajian, K. N., et al. 2009, ApJS, 182, 543

Adelman-McCarthy, J. K., et al. 2006, ApJS, 162, 38

Adelman-McCarthy, J. K., et al. 2008, ApJS, 175, 297

Allende Prieto, C., Barklem, P. S., Lambert, D. L., \& Cunha, K. 2004, A\&A, 420,183

Allende Prieto, C., Beers, T. C., Wilhelm, R., Newberg, H. J., Rockosi, C. M., Yanny, B., \& Lee, Y. S. 2006, ApJ, 636, 804

Allende Prieto, C., et al. 2008, AJ, 136, 2070

Amendt, P., \& Cuddeford, P. 1991, ApJ, 368, 79

An, D., et al. 2009, ApJ, 707, L64

Battaglia, G., et al. 2005, MNRAS, 364, 433

Beers, T. C., et al. 2006, Mem. Soc. Astron. Ital., 77, 1171

Binney, J., \& Merrifield, M. (eds.) 1998, Galactic Astronomy (Princeton, NJ: Princeton Univ. Press)

Binney, J., \& Tremaine, S. 1987, Galactic Dynamics (Princeton, NJ: Princeton Univ. Press)

Bochanski, J. J., Munn, J. A., Hawley, S. L., West, A. A., Covey, K. R., \& Schneider, D. P. 2007a, AJ, 134, 2418

Bochanski, J. J., West, A. A., Hawley, S. L., \& Covey, K. R. 2007b, AJ, 133, 531

Bovy, J., Hogg, D. W., \& Rix, H.-W. 2009, ApJ, 704, 1704

Bramich, D. M., et al. 2008, MNRAS, 386, 887

Carlberg, R. G., \& Innanen, K. A. 1987, AJ, 94, 666

Carollo, D., et al. 2007, Nature, 450, 1020

Carollo, D., et al. 2009, ApJ, submitted (arXiv:0909.3019)

Chiba, M., \& Beers, T. C. 2000, AJ, 119, 2843 
De Simone, R., Wu, X., \& Tremaine, S. 2004, MNRAS, 350, 627

Dehnen, W. 1998, AJ, 115, 2384

Dehnen, W., \& Binney, J. J. 1998, MNRAS, 298, 387

Eggen, O. J. 1996, AJ, 112, 1595

Eggen, O. J., Lynden-Bell, D., \& Sandage, A. R. 1962, ApJ, 136, 748

Eisenhauer, F., Schödel, R., Genzel, R., Ott, T., Tecza, M., Abuter, R., Eckart, A., \& Alexander, T. 2003, ApJ, 597, L121

Famaey, B., Jorissen, A., Luri, X., Mayor, M., Udry, S., Dejonghe, H., \& Turon, C. $2005, A \& A, 430,165$

Feast, M. W., \& Whitelock, P. A. 1997, in Proc. ESA Symp. 402, HipparcosVenice '97 (ESA Special Publication, Noordwijk: ESA), 625

Flaugher, B. 2008, in Proc. Conf., A Decade of Dark Energy: Spring Symp., ed. Norbert Pirzkal \& Henry Ferguson (Baltimore, MD: STSci), (http://www.stsci.edu/institute/conference/spring2008)

Fuchs, B., et al. 2009, AJ, 137, 4149

Fukugita, M., Ichikawa, T., Gunn, J. E., Doi, M., Shimasaku, K., \& Schneider, D. P. 1996, AJ, 111, 1748

Gilmore, G., Wyse, R. F. G., \& Kuijken, K. 1989, ARA\&A, 27, 555

Grillmair, C. J., \& Dionatos, O. 2006, ApJ, 643, L17

Gunn, J. E., Knapp, G. R., \& Tremaine, S. D. 1979, AJ, 84, 1181

Gunn, J. E., et al. 1998, AJ, 116, 3040

Gunn, J. E., et al. 2006, AJ, 131, 2332

Helmi, A. 2008, A\&AR, 15, 145

Hogg, D. W., Blanton, M. R., Roweis, S. T., \& Johnston, K. V. 2005, ApJ, 629, 268

Hogg, D. W., Finkbeiner, D. P., Schlegel, D. J., \& Gunn, J. E. 2001, AJ, 122, 2129

Holmberg, J., Nordström, B., \& Andersen, J. 2007, A\&A, 475, 519

Holmberg, J., Nordström, B., \& Andersen, J. 2009, A\&A, 501, 941

Ivezić, Ž., et al. 2004, Astron. Nachr., 325, 583

Ivezić, Ž., et al. 2005, in ASP Conf. Ser. 338, Astrometry in the Age of the Next Generation of Large Telescopes, ed. P. K. Seidelmann \& A. K. B. Monet (San Francisco, CA: ASP), 201

Ivezić, Ž., et al. 2006, Mem. Soc. Astron. Ital., 77, 1057

Ivezić, Ž., et al. 2008a, ApJ, 684, 287

Ivezić, Ž., et al. 2008b, arXiv:0805.2366

Jurić, M., et al. 2008, ApJ, 673, 864

Kaczmarczik, M. C., Richards, G. T., Mehta, S. S., \& Schlegel, D. J. 2009, AJ, 138,19

Kaiser, N., et al. 2002, Proc. SPIE, 4836, 154

Kent, S. M., \& de Zeeuw, T. 1991, AJ, 102, 1994

Klement, R., et al. 2009, ApJ, 698, 865
Lee, Y. S., \& Beers, T. C. 2009, BAAS, 41, 227

Lee, Y. S., et al. 2008a, AJ, 136, 2022

Lee, Y. S., et al. 2008b, AJ, 136, 2050

Loebman, S., et al. 2008, in AIP Conf. Ser. 1082, Classification and Discovery in Large Astronomical Surveys, ed. C. A. L. Bailer-Jones (Melville, NY: AIP), 238

Majewski, S. R. 1992, ApJS, 78, 87

Majewski, S. R. 1993, ARA\&A, 31, 575

Munn, J. A., et al. 2004, AJ, 127, 3034

Munn, J. A., et al. 2008, AJ, 136, 895

Murray, C. A. 1986, MNRAS, 223, 649

Nordström, B., et al. 2004, A\&A, 418, 989

Perryman, M. A. C., et al. 2001, A\&A, 369, 339

Pier, J. R., Munn, J. A., Hindsley, R. B., Hennessy, G. S., Kent, S. M., Lupton, R. H., \& Ivezić, Ž. 2003, AJ, 125, 1559

Prugniel, P., Soubiran, C., Koleva, M., \& Le Borgne, D. 2007, VizieR Online Data Catalog, 3251, 0

Robin, A., \& Creze, M. 1986, A\&A, 157, 71

Robin, A. C., Reylé, C., Derrière, S., \& Picaud, S. 2003, A\&A, 409, 523

Rocha-Pinto, H. J., Flynn, C., Scalo, J., Hänninen, J., Maciel, W. J., \& Hensler, G. 2004, A\&A, 423, 517

Roškar, R., Debattista, V. P., Quinn, T. R., Stinson, G. S., \& Wadsley, J. 2008, ApJ, 684, L79

Schlaufman, K. C., et al. 2009, ApJ, 703, 2177

Schlegel, D. J., Finkbeiner, D. P., \& Davis, M. 1998, ApJ, 500, 525

Schneider, D. P., et al. 2007, AJ, 134, 102

Schönrich, R., \& Binney, J. 2009, MNRAS, 399, 1145

Schwarzschild, M. 1979, ApJ, 232, 236

Sesar, B., Ivezić, Ž., \& Jurić, M. 2008, ApJ, 689, 1244

Siebert, A., et al. 2008, MNRAS, 391, 793

Sirko, E., et al. 2004, AJ, 127, 914

Smith, J. A., et al. 2002, AJ, 123, 2121

Smith, M. C., et al. 2009, MNRAS, 399, 1223

Stoughton, C., et al. 2002, AJ, 123, 485

Tucker, D. L., et al. 2006, Astron. Nachr., 327, 821

West, A. A., Hawley, S. L., Bochanski, J. J., Covey, K. R., Reid, I. N., Dhital, S., Hilton, E. J., \& Masuda, M. 2008, AJ, 135, 785

Wilkinson, M. I., et al. 2005, MNRAS, 359, 1306

Xue, X. X., et al. 2008, ApJ, 684, 1143

Yanny, B., et al. 2009, AJ, 137, 4377

York, D. G., et al. 2000, AJ, 120, 1579 